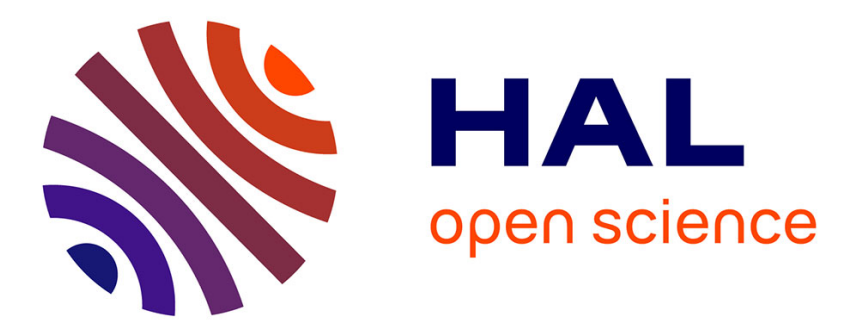

\title{
Bounds for multivariate residues and for the polynomials in the elimination theorem
}

\author{
Martin Sombra, Alain Yger
}

\section{To cite this version:}

Martin Sombra, Alain Yger. Bounds for multivariate residues and for the polynomials in the elimination theorem. Moscow Mathematical Journal, In press. hal-02879624

\section{HAL Id: hal-02879624 \\ https://hal.science/hal-02879624}

Submitted on 24 Jun 2020

HAL is a multi-disciplinary open access archive for the deposit and dissemination of scientific research documents, whether they are published or not. The documents may come from teaching and research institutions in France or abroad, or from public or private research centers.
L'archive ouverte pluridisciplinaire HAL, est destinée au dépôt et à la diffusion de documents scientifiques de niveau recherche, publiés ou non, émanant des établissements d'enseignement et de recherche français ou étrangers, des laboratoires publics ou privés. 


\title{
BOUNDS FOR MULTIVARIATE RESIDUES AND FOR THE POLYNOMIALS IN THE ELIMINATION THEOREM
}

\author{
MARTÍN SOMBRA AND ALAIN YGER
}

\begin{abstract}
We present several upper bounds for the height of global residues of rational forms on an affine variety defined over $\mathbb{Q}$. As an application, we deduce upper bounds for the height of the coefficients in the Bergman-Weil trace formula.

We also present upper bounds for the degree and the height of the polynomials in the elimination theorem on an affine variety defined over $\mathbb{Q}$. This is an arithmetic analogue of Jelonek's effective elimination theorem, and it plays a crucial role in the proof of our bounds for the height of global residues.
\end{abstract}

\section{Contents}

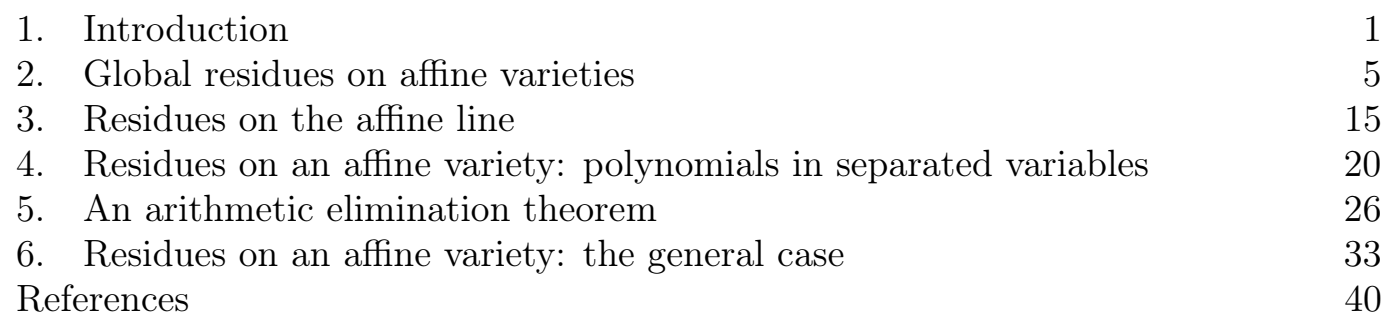

\section{INTRODUCTION}

Given a regular sequence $\boldsymbol{f}=\left(f_{1}, \ldots, f_{n}\right)$ in the $n$-dimensional local ring $\mathscr{O}_{\mathbb{C}^{n}, 0}$ of germs at 0 of holomorphic functions of $\mathbb{C}^{n}$ and $\omega=g \mathrm{~d} x_{1} \wedge \cdots \wedge \mathrm{d} x_{n}$ a germ at 0 of a holomorphic $n$-form on $\mathbb{C}^{n}$, the Grothendieck local residue at 0 of $\omega$ with respect to $f$ is defined as

$$
\operatorname{Res}_{0}\left[\begin{array}{l}
\omega \\
\boldsymbol{f}
\end{array}\right]=\frac{1}{(2 i \pi)^{n}} \int_{\left|f_{1}\right|=\varepsilon_{1}, \ldots,\left|f_{n}\right|=\varepsilon_{n}} \frac{g \mathrm{~d} x_{1} \wedge \cdots \wedge \mathrm{d} x_{n}}{f_{1} \cdots f_{n}}
$$

for $\varepsilon=\left(\varepsilon_{1}, \ldots, \varepsilon_{n}\right) \in\left(\mathbb{R}_{\geq 0}\right)^{n}$ sufficiently small.

This notion can be transposed to the more general situation of an $r$-dimensional local ring $\mathscr{O}_{X, \boldsymbol{x}_{0}}$ for a (reduced) complex space $X$ of pure dimension $r \geq 1, \boldsymbol{f}=\left(f_{1}, \ldots, f_{r}\right)$ a regular sequence in $\mathscr{O}_{X, \boldsymbol{x}_{0}}$, and $\omega$ a germ at $\boldsymbol{x}_{0}$ of a holomorphic $n$-form on $X$, thus leading to the definition of the local residue, denoted as

$$
\operatorname{Res}_{X, x_{0}}\left[\begin{array}{l}
\omega \\
f
\end{array}\right] \text {. }
$$

When $X \subset \mathbb{C}^{n}$ is an algebraic variety of pure dimension $r \geq 1, \boldsymbol{f}=\left(f_{1}, \ldots, f_{r}\right)$ a family of polynomials in $\mathbb{C}\left[x_{1}, \ldots, x_{n}\right]$ defining a complete intersection on $X$, and $\omega$ a rational

Date: June 24, 2020.

2010 Mathematics Subject Classification. Primary 32A27; Secondary 11G50, 14 Q20.

Key words and phrases. Residues, membership problems, height.

Sombra was partially supported by the MINECO research projects MTM2012-38122-C03-02 and MTM2015-65361-P. Yger was partially supported by the CNRS research project PICS 6381 "Géométrie diophantienne et calcul formel". 
$r$-form on $\mathbb{C}^{n}$ that is regular on $X \cap V(\boldsymbol{f})$, the zero set of the system $\boldsymbol{f}$ on $X$, the (global) residue on $X$ of $\omega$ with respect to $f$ might be defined as of the total sum the local residues at the points of $X \cap V(\boldsymbol{f})$, that is

$$
\operatorname{Res}_{X}\left[\begin{array}{l}
\omega \\
\boldsymbol{f}
\end{array}\right]=\sum_{\boldsymbol{x}_{0} \in X \cap V(\boldsymbol{f})} \operatorname{Res}_{X, \boldsymbol{x}_{0}}\left[\begin{array}{l}
\omega \\
\boldsymbol{f}
\end{array}\right] .
$$

These residues play an important role in division formulae on polynomial rings. An example of these is the Bergman-Weil trace formula for the case when $X=\mathbb{C}^{n}$ AY83, Tsi92, BH99. This formula was the key tool towards the first versions of the arithmetic Nullstellensatz, giving bounds for the degree and the height of the polynomials in the Nullstellensatz [BGVY94, BY99]. Global residues have also a deep connection with duality for algebraic varieties [Kun08 and, moreover, they appear in several fields of mathematics and theoretical physics, like scattering amplitude computations beyond Feynman diagrams SZ16.

Many of these applications involve not just one residue, but the whole residue multisequence associated to the triple $(X, \boldsymbol{f}, \omega)$, given by

$$
\left(\operatorname{Res}_{X}\left[\begin{array}{c}
\omega \\
f^{\boldsymbol{\alpha}+\mathbf{1}}
\end{array}\right]\right)_{\boldsymbol{\alpha} \in \mathbb{N}^{n}}
$$

with $\boldsymbol{f}^{\boldsymbol{\alpha}+\mathbf{1}}:=\left(f_{1}^{\alpha_{1}+1}, \ldots, f_{r}^{\alpha_{r}+1}\right) \in \mathbb{C}\left[x_{1}, \ldots, x_{n}\right]^{r}$ for $\boldsymbol{\alpha} \in \mathbb{N}^{n}$. For instance, this multisequence is relevant for the Bergman-Weil formula and for the representation of traces in terms of residues, as explained in $\$ 2.2$.

In the arithmetic setting, that is, when the triple $(X, \boldsymbol{f}, \omega)$ is defined over the field of rational numbers, the corresponding residues are rational numbers. In spite of their applications, up to our knowledge there are no available results allowing to control the arithmetic complexity or height of global residues, that is, to bound the numerator and denominator of these rational numbers. It is precisely our aim in this paper to provide this kind of bounds.

To state our results, suppose for the rest of this introduction that $X$ is a subvariety of $\mathbb{A}_{\mathbb{Q}}^{n}$ of pure dimension $r, \boldsymbol{f}$ a system of nonconstant polynomials with integer coefficients defining a complete intersection on $X$, and $\omega$ a rational $r$-form defined over $\mathbb{Q}$ that is regular on $X \cap V(\boldsymbol{f})$. For $\boldsymbol{\alpha} \in \mathbb{N}^{n}$, write the corresponding global residue as

$$
\operatorname{Res}_{X_{\mathbb{C}}}\left[\begin{array}{c}
\omega \\
f^{\alpha+1}
\end{array}\right]=\frac{a_{\boldsymbol{\alpha}}}{b_{\boldsymbol{\alpha}}}
$$

with $a_{\boldsymbol{\alpha}} \in \mathbb{Z}$ and $b_{\boldsymbol{\alpha}} \in \mathbb{Z} \backslash\{0\}$ coprime.

For a polynomial $f \in \mathbb{Z}\left[x_{1}, \ldots, x_{n}\right]$, its height, denoted by $\mathrm{h}(f)$, is the logarithm of the maximum of the absolute value of its coefficients. A polynomial $r$-form $\tau$ on $\mathbb{C}^{n}$ defined over $\mathbb{Z}$ is a holomorphic $r$-form that writes down as

$$
\tau=\sum_{I} g_{I} \mathrm{~d} \boldsymbol{x}_{I},
$$

the sum being over the subsets $I \subset\{1, \ldots, n\}$ of cardinality $r$, with $g_{I} \in \mathbb{Z}\left[x_{1}, \ldots, x_{n}\right]$ and

$$
\mathrm{d} \boldsymbol{x}_{I}=\bigwedge_{j=1}^{r} \mathrm{~d} x_{i_{j}} .
$$

for $1 \leq i_{1}<\cdots<i_{r} \leq n$ such that $I=\left\{i_{1}, \ldots, i_{r}\right\}$. The degree and the length of $\tau$, denoted by $\operatorname{deg}(\tau)$ and by $\mathrm{h}_{1}(\tau)$, are respectively defined as the maximum degree of the $g_{I}$ 's and as the logarithm of the $\ell^{1}$-norm of the coefficient list of all the $g_{I}$ 's. 
The degree and height of the subvariety $X$, denoted by $\operatorname{deg}(X)$ and by $\mathrm{h}(X)$, are respectively defined as the degree and the canonical height of the closure of its image with respect to the standard inclusion $\mathbb{A}_{\mathbb{Q}}^{n} \hookrightarrow \mathbb{P}_{\mathbb{Q}}^{n}$. The degree and the height of an affine variety are measures of its geometric and arithmetic complexity, see for instance [KPS01, §1.2] or [DKS13, §2.3] for more details.

The following result corresponds to Theorem 6.6 , and bounds the numerator and denominators in the residue multi-sequence (1.1) in the most general situation considered in this paper.

Theorem 1.1. With notation as above, write $\omega=\tau / f_{0}$ with $\tau$ a polynomial $r$-form on $\mathbb{C}^{n}$ defined over $\mathbb{Z}$ and $f_{0} \in \mathbb{Z}\left[x_{1}, \ldots, x_{n}\right]$ not vanishing on $X \cap V(\boldsymbol{f})$. Set $d_{i}=\operatorname{deg}\left(f_{i}\right)$, $i=0, \ldots, r$, and $e=\operatorname{deg}(\tau)$. Set also

$D_{X, \boldsymbol{f}}=\operatorname{deg}(X) \prod_{j=1}^{r} d_{j}$ and $\kappa_{X, \boldsymbol{f}}=\frac{\mathrm{h}(X)}{\operatorname{deg}(X)}+\sum_{j=1}^{r} \frac{\mathrm{h}\left(f_{j}\right)}{d_{j}}+4(n+5)^{2} \log ((n+1) \operatorname{deg}(X))$.

Then, for $\boldsymbol{\alpha} \in \mathbb{N}^{r}$,

$$
\begin{aligned}
\log \left|a_{\boldsymbol{\alpha}}\right|, \log \left|b_{\boldsymbol{\alpha}}\right| & +\mathrm{h}_{1}(\tau) \leq\left(\begin{array}{c}
n \\
r
\end{array}\right)\left(\mathrm{h}_{1}(\tau)+e(r+1) D_{X, \boldsymbol{f}} \kappa_{X, \boldsymbol{f}}\right. \\
& \left.+(|\boldsymbol{\alpha}|+1)\left(2(r+1) D_{X, \boldsymbol{f}} \mathrm{h}\left(f_{0}\right)+\left(3 d_{0}+r+1\right) D_{X, \boldsymbol{f}}^{2} \kappa_{X, \boldsymbol{f}}\right)\right) .
\end{aligned}
$$

In Theorem 6.7. we give a sharper bound for the case when $X=\mathbb{A}_{\mathbb{Q}}^{n}$ : set now

$$
D_{\boldsymbol{f}}=\prod_{j=1}^{n} d_{j} \quad \text { and } \quad \kappa_{\boldsymbol{f}}^{\prime}=\sum_{j=1}^{n} \frac{\mathrm{h}\left(f_{j}\right)}{d_{j}}+(4 n+8) \log (n+3) .
$$

Then, for $\boldsymbol{\alpha} \in \mathbb{N}^{n}$,

$$
\begin{aligned}
\log \left|a_{\boldsymbol{\alpha}}\right|, \log \left|b_{\boldsymbol{\alpha}}\right|+\mathrm{h}_{1}(\tau) \leq \mathrm{h}_{1}(\tau) & +e n D_{\boldsymbol{f}} \kappa_{\boldsymbol{f}}^{\prime} \\
& +(|\boldsymbol{\alpha}|+1)\left(D_{\boldsymbol{f}} \mathrm{h}\left(f_{0}\right)+\left(d_{0}+n\right)\left(n D_{\boldsymbol{f}}+1\right) D_{\boldsymbol{f}} \kappa_{\boldsymbol{f}}^{\prime}\right) .
\end{aligned}
$$

Also, in Theorems 6.2 and 6.3 we present sharper bounds for the special case when $X$ is in good position with respect to the system of coordinates, in the sense that $\# X \cap V\left(x_{1}, \ldots, x_{r}\right)=\operatorname{deg}(X)$, and $\omega$ is a polynomial $r$-form. Nevertheless, all of these bounds have a quadratic dependence on the Bézout number $D_{X, f}$ that does not seem optimal, although at this moment we cannot not tell if this is the case or not.

For the affine line $X=\mathbb{A}_{\mathbb{Q}}^{1}$, residue calculus and Euclidean division are deeply correlated. The following result, corresponding to Theorem 3.2, bounds the numerators and denominators of the residue sequence of to a polynomial 1-form on the affine line.

Theorem 1.2. Let $f \in \mathbb{Z}[x] \backslash \mathbb{Z}$ and $g \in \mathbb{Z}[x]$. Set $d=\operatorname{deg}(f)$ and $e=\operatorname{deg}(g)$, and let $f_{d}$ be the leading coefficient of $f$. Then, for $\alpha \in \mathbb{N}$,

$$
f_{d}^{e+1-(\alpha+1)(d-1)} \operatorname{Res}\left[\begin{array}{l}
g \mathrm{~d} x \\
f^{\alpha+1}
\end{array}\right] \in \mathbb{Z}
$$

and

$\log \left|f_{d}^{e+1-(\alpha+1)(d-1)} \operatorname{Res}\left[\begin{array}{l}g \mathrm{~d} x \\ f^{\alpha+1}\end{array}\right]\right| \leq \mathrm{h}_{1}(g)+(e+1-(\alpha+1) d) \mathrm{h}(f)+(e-d+1) \log (2)$.

If $e<(\alpha+1) d-1$, then $\operatorname{Res}\left[\begin{array}{l}g \mathrm{~d} x \\ f^{\alpha+1}\end{array}\right]=0$. 
In Theorems 4.3 and 4.4 , we generalize this result to residues on a higher dimensional affine variety and a system of univariate polynomials in separated variables. In contrast to the general case, these upper bounds do seem to be sharp, as shown by Example 3.6 for the case of the affine line.

Our approach to these results is based on the arithmetic membership problem that we next explain, since it might be of independent interest. Recall that $X \subset \mathbb{A}_{\mathbb{Q}}^{n}$ is a variety of pure dimension $r$ and $\boldsymbol{f}=\left(f_{1}, \ldots, f_{r}\right)$ a system of polynomials in $\mathbb{Z}\left[x_{1}, \ldots, x_{n}\right] \backslash \mathbb{Z}$ with a finite number of common zeros in $X$. A classical method to solve the system of equations given by

$$
f_{1}(\boldsymbol{x})=\cdots=f_{r}(\boldsymbol{x})=0 \quad \text { for } \boldsymbol{x} \in X
$$

is to eliminate variables, that is, find $\phi_{l} \in \mathbb{Z}\left[x_{l}\right] \backslash\{0\}, l=1, \ldots, r$, and $a_{l, i} \in$ $\mathbb{Z}\left[x_{1}, \ldots, x_{n}\right], l, i=1, \ldots, n$, such that

$$
\phi_{l}=\sum_{i=1}^{n} a_{l, i} f_{i} \quad \text { on } X,
$$

in the sense that this polynomial relation holds modulo the ideal of definition of $X$.

In [Jel05, Jelonek obtained an optimal upper bound for the degrees of these polynomials, using a variant of his approach to the effective Nullstellensatz. Here we prove the following arithmetic analogue of this result, corresponding to Corollary 5.6 in the body of the paper. Its proof proceeds by adapting Jelonek's approach with the tools from geometric and arithmetic intersection theory from [DKS13].

Theorem 1.3. With notation as above, there are $\phi_{l} \in \mathbb{Z}\left[x_{l}\right] \backslash\{0\}$ and $a_{l, 1}, \ldots, a_{l, r} \in$ $\mathbb{Z}\left[x_{1}, \ldots, x_{n}\right], l, i=1, \ldots, n$, satisfying the identity (1.4) with

$$
\begin{aligned}
& \operatorname{deg}\left(\phi_{l}\right), \operatorname{deg}\left(a_{l, i}\right)+d_{i} \leq\left(\prod_{j=1}^{r} d_{j}\right) \operatorname{deg}(X), \\
& \mathrm{h}\left(\phi_{l}\right), \mathrm{h}\left(a_{l, i}\right)+\mathrm{h}\left(f_{i}\right) \leq\left(\prod_{j=1}^{r} d_{j}\right)\left(\mathrm{h}(X)+\operatorname{deg}(X)\left(\sum_{j=1}^{r} \frac{\mathrm{h}\left(f_{j}\right)}{d_{j}}\right.\right. \\
&\left.\left.+(r+1) \log \left(2(r+2)(n+1)^{2}\right)\right)\right) .
\end{aligned}
$$

In the case $X=\mathbb{A}_{\mathbb{Q}}^{n}$, we have $r=n, \operatorname{deg}\left(\mathbb{A}_{\mathbb{Q}}^{n}\right)=1$ and $\mathrm{h}\left(\mathbb{A}_{\mathbb{Q}}^{n}\right)=0$. If moreover $\operatorname{deg}\left(f_{j}\right) \leq d$ and $\mathrm{h}\left(f_{j}\right) \leq h$ for all $j$, then the previous bound specializes to

$$
\begin{aligned}
& \operatorname{deg}\left(\phi_{l}\right), \operatorname{deg}\left(a_{l, i}\right)+\operatorname{deg}\left(f_{i}\right) \leq d^{n}, \\
& \mathrm{~h}\left(\phi_{l}\right), \mathrm{h}\left(a_{l, i}\right)+\mathrm{h}\left(f_{i}\right) \leq n d^{n-1} h+(n+2) \log \left(2(n+2)(n+1)^{2}\right) d^{n} .
\end{aligned}
$$

The proof of Theorem 1.1 and 6.7 is incremental. We first obtain the bounds for residues on the affine line (Theorems 1.2 and 3.10 by a recurrence scheme based on the relationship of these residues with the Euclidean division. The treatment of residues on affine varieties and a system of univariate polynomials in separated variables (Theorems 4.3 and 4.4 is based on the arithmetic Perron's theorem from DKS13 and the relationship between residues and traces on polynomial algebras. The general case is then treated by reducing to the case of univariate polynomials by applying the transformation law (Theorem 2.8) and Theorem 1.3 .

As an application of our results on the heights in the residue multi-sequence, we derive a bound for the coefficients in the Bergman-Weil trace formula. To formulate it, let $\boldsymbol{f}=\left(f_{1}, \ldots, f_{n}\right)$ be a family of polynomials in $\mathbb{Z}\left[x_{1}, \ldots, x_{n}\right] \backslash \mathbb{Z}$ defining a complete intersection on $\mathbb{A}_{\mathbb{Q}}^{n}$ and, for simplicity, suppose that the map $\mathbb{A}_{\mathbb{Q}}^{n} \rightarrow \mathbb{A}_{\mathbb{Q}}^{n}$ 
defined by $\boldsymbol{x} \mapsto \boldsymbol{f}(\boldsymbol{x})$ is proper. Set $\boldsymbol{d}=\left(d_{1}, \ldots, d_{n}\right) \in \mathbb{N}^{n}$ with $d_{i}=\operatorname{deg}\left(f_{i}\right)$. For $p \in \mathbb{Z}\left[x_{1}, \ldots, x_{n}\right]$, the Bergman-Weil trace formula gives an explicit polynomial identity

$$
p=\sum_{\alpha \in \mathbb{N}^{n}} p_{\alpha} f^{\alpha}
$$

with $p_{\boldsymbol{\alpha}} \in \mathbb{Q}\left[x_{1}, \ldots, x_{n}\right]$ of degree bounded by $|\boldsymbol{d}|-n$, that are zero except for a finite number of $\boldsymbol{\alpha}$ 's (Theorem 2.15) . The next result corresponds to Corollary 6.4

Theorem 1.4. With notation as above, set

$$
D_{\boldsymbol{f}}=\prod_{j=1}^{n} d_{j} \quad \text { and } \quad \kappa_{\boldsymbol{f}}^{\prime \prime}=\sum_{j=1}^{n} \frac{\mathrm{h}\left(f_{j}\right)}{d_{j}}+3(n+2) \log (n+2) .
$$

Set also $e=\operatorname{deg}(p)$. Then there exists $\vartheta \in \mathbb{Z} \backslash\{0\}$ with $\log |\vartheta| \leq n \kappa_{\boldsymbol{f}}^{\prime \prime}$ such that, for $\boldsymbol{\alpha} \in \mathbb{N}^{n}$, we have that $\vartheta^{e+|\boldsymbol{d}|+(|\boldsymbol{\alpha}|+1)\left(n D_{\boldsymbol{f}}+1\right)} p_{\boldsymbol{\alpha}} \in \mathbb{Z}\left[x_{1}, \ldots, x_{n}\right]$ and

$$
\mathrm{h}\left(\vartheta^{e+|\boldsymbol{d}|+(|\boldsymbol{\alpha}|+1)\left(n D_{\boldsymbol{f}}+1\right)} p_{\boldsymbol{\alpha}}\right) \leq \mathrm{h}_{1}(g)+\left(e+|\boldsymbol{d}|+(|\boldsymbol{\alpha}|+1)\left(n D_{\boldsymbol{f}}+1\right)\right) n D_{\boldsymbol{f}} \kappa_{\boldsymbol{f}}^{\prime \prime} .
$$

In a general way, we expect that the result from this paper might be useful to obtain arithmetic versions of other problems from effective commutative algebra allowing an analytic treatment, like the Briançon-Skoda theorem or the Artin-Rees lemma, among others.

The paper is organized as follows. In $\$ 2$, we recall the definition of the global residue in the algebraic setting and its basic properties, including the transformation law and other results from multivariate residue calculus. In \$3, we study in detail global residues on the affine line. Section 4 is devoted to the case of an arbitrary affine variety and univariate polynomials in separated variables. In $\$ 5$, we present the arithmetic analogue of Jelonek's theorem, bounding the degree and the height of the polynomials in the elimination theorem. Finally, in w6 we exploit these arithmetic constructions in accordance with multivariate residue calculus as described in $\$ 2$, to achieve the stated bounds for the height of multivariate residues.

Acknowledgments. Part of this work was done while the authors met at the Universitat de Barcelona and at the Institut de Mathématiques de Bordeaux. We thank these institutions for their hospitality.

\section{Global Residues on AfFine varieties}

In this section, we introduce global residues of meromorphic forms on affine varieties and recall its basic properties. This material is classical, and we base most of our exposition on the book [CH78] and on the paper [BVY05]. We refer to these sources for precisions and the proof of the stated results.

Boldface letters and symbols denote finite sets or sequences of objects, where the type and number should be clear from the context: for instance, for $n \geq 1$ we denote by $\boldsymbol{x}$ the group of variables $\left(x_{1}, \ldots, x_{n}\right)$, so that $\mathbb{C}[\boldsymbol{x}]=\mathbb{C}\left[x_{1}, \ldots, x_{n}\right]$.

2.1. Definition and basic properties. Let $X \subset \mathbb{A}_{\mathbb{C}}^{n}$ be a variety of pure dimension $r \geq 1$ and $\boldsymbol{f}=\left(f_{1}, \ldots, f_{r}\right) \in \mathbb{C}\left[x_{1}, \ldots, x_{n}\right]^{r}=\mathbb{C}[\boldsymbol{x}]^{r}$ a family of $r$ polynomials in $n$ variables defining a complete intersection on $X$. To simplify the exposition, we identify $X$ with its set of complex points $X(\mathbb{C})$.

We denote by $X^{\text {sing }}$ and $X^{\text {reg }}$ the subsets of $X$ of singular and regular points, respectively. Since the family $\boldsymbol{f}$ defines a complete intersection on $X$, its Jacobian 
locus is proper closed subset of $X$. We denote by $W$ an algebraic hypersurface of $X$ containing both $X^{\text {sing }}$ and this Jacobian locus. We also denote by

$$
X \cap V(\boldsymbol{f})=\left\{\boldsymbol{x} \in X \mid f_{1}(\boldsymbol{x})=\cdots=f_{r}(\boldsymbol{x})=0\right\}
$$

the finite set of zeros of the system $\boldsymbol{f}$ on $X$.

Let $\|\cdot\|$ denote the Euclidean norm of $\mathbb{C}^{n}$ and fix $R>0$ such that the open ball

$$
B_{R}=\left\{\boldsymbol{x} \in \mathbb{C}^{n} \mid\|x\|<R\right\}
$$

contains $X \cap V(\boldsymbol{f})$. Let $\eta>0$ and $\varepsilon=\left(\varepsilon_{1}, \ldots, \varepsilon_{r}\right) \in\left(\mathbb{R}_{\geq 0}\right)^{n}$ with $\varepsilon_{i} \leq \eta$ for all $i$, and consider the tube around $X \cap V(\boldsymbol{f})$ given by

$$
\Gamma_{\boldsymbol{\varepsilon}}=B_{R} \cap\left\{\boldsymbol{x} \in X|| f_{1}(\boldsymbol{x})\left|=\varepsilon_{1}, \ldots,\right| f_{r}(\boldsymbol{x}) \mid=\varepsilon_{r}\right\} .
$$

When $\eta$ is sufficiently small, this is a compact, not necessarily connected, semianalytic set of dimension $r$, without components contained in $W$ and smooth outside this hypersurface. We orient the smooth semianalytic set $\Gamma_{\varepsilon} \backslash W$ so that the inverse image to it of the differential $r$-form $\bigwedge_{j=1}^{r} \operatorname{darg}\left(f_{j}\right)$ is positive.

Let $\omega$ be a meromorphic $r$-form on $\mathbb{C}^{n}$ that is regular on $X \cap V(\boldsymbol{f})$. For $\boldsymbol{\alpha}=$ $\left(\alpha_{1}, \ldots, \alpha_{r}\right) \in \mathbb{N}^{r}$, the integral

$$
\int_{\Gamma_{\varepsilon}} \frac{\omega}{f_{1}^{\alpha_{1}+1} \cdots f_{r}^{\alpha_{r}+1}}
$$

is defined as the integral of a regular $(r, 0)$-form on the $r$-dimensional smooth semianalytic chain $\Gamma_{\varepsilon} \backslash W$. Its value does not depend on the choice of $W$. It does neither depend on the choice of $\varepsilon$, by Stokes' theorem on semianalytic chains ([Her66, B-§2,3], Poly74).

Definition 2.1. Let $X \subset \mathbb{A}_{\mathbb{C}}^{n}$ be a variety of pure dimension $r \geq 1, \boldsymbol{f}=\left(f_{1}, \ldots, f_{r}\right) \in$ $\mathbb{C}[\boldsymbol{x}]^{r}$ a family of $r$ polynomials defining a complete intersection on $X$, and $\omega$ a meromorphic $r$-form on $\mathbb{C}^{n}$ that is regular on $X \cap V(\boldsymbol{f})$. With notation as above, given $\boldsymbol{\alpha} \in \mathbb{N}^{r}$, the (global) residue on $X$ of $\omega$ with respect to $\boldsymbol{f}^{\boldsymbol{\alpha}+\mathbf{1}}:=\left(f_{1}^{\alpha_{1}+1}, \ldots, f_{r}^{\alpha_{r}+1}\right)$ is defined as

$$
\operatorname{Res}_{X}\left[\begin{array}{c}
\omega \\
f^{\boldsymbol{\alpha}+\mathbf{1}}
\end{array}\right]=\frac{1}{(2 \pi i)^{r}} \int_{\Gamma_{\varepsilon}} \frac{\omega}{f_{1}^{\alpha_{1}+1} \cdots f_{r}^{\alpha_{r}+1}}
$$

for any $\eta>0$ sufficiently small and $\varepsilon=\left(\varepsilon_{1}, \ldots, \varepsilon_{r}\right) \in\left(\mathbb{R}_{\geq 0}\right)^{n}$ with $\varepsilon_{i} \leq \eta$ for all $i$.

Remark 2.2. This notion coincides with that in (1.1). Since in this paper we are only concerned with global residues, here we define them directly without passing through the local case.

To profit from the flexibility of analysis, as well as to emphasize the action of the residue instead of the result of this action on an specific form, it is often convenient to enlarge this notion with a currential approach. Following Coleff and Herrera [CH78, $\S 4.1$, we can define a residual current by considering the limit of residue integrals along special, so-called "admissible", paths of the form

$$
s \mapsto \varepsilon(s)=\left(s^{\beta_{1}}, \ldots, s^{\beta_{r}}\right)
$$

for some fixed positive numbers $\beta_{1} \gg \cdots \gg \beta_{r}$. Given a compactly supported $(r, 0)$ form $\eta$ and $\boldsymbol{\alpha} \in \mathbb{N}^{r}$, the limit

$$
\left\langle\bigwedge_{j=1}^{r} \bar{\partial}\left(\frac{1}{f_{j}^{\alpha_{j}+1}}\right) \wedge[X], \eta\right\rangle=\lim _{s \rightarrow 0} \frac{1}{(2 \pi i)^{r}} \int_{\Gamma_{\varepsilon(s)}} \frac{\eta}{f_{1}^{\alpha_{1}+1} \cdots f_{r}^{\alpha_{r}+1}}
$$


exists and defines a $(0, r)$-current which is independent of the choice of the admissible path [CH78]. Using an arbitrary $C^{\infty}$-function $\chi: \mathbb{C}^{n} \rightarrow \mathbb{R}$ with compact support that is identically equal to 1 on a neighborhood of $X \cap V(\boldsymbol{f})$, the residues in Definition 2.1 can be then written as

$$
\operatorname{Res}_{X}\left[\begin{array}{c}
\omega \\
f^{\boldsymbol{\alpha}+\mathbf{1}}
\end{array}\right]=\left\langle\bigwedge_{j=1}^{r} \bar{\partial}\left(\frac{1}{f_{j}^{\alpha_{j}+1}}\right) \wedge[X], \chi \omega\right\rangle .
$$

Residues of holomorphic $r$-forms can also be represented as integrals of the BochnerMartinelli type over a $(2 r-1)$-dimensional cycle. For $\boldsymbol{\alpha} \in \mathbb{N}^{r}$, we set

$$
|\boldsymbol{\alpha}|=\alpha_{1}+\cdots+\alpha_{r} \quad \text { and } \quad \boldsymbol{\alpha} !=\alpha_{1} ! \ldots \alpha_{r} !
$$

Proposition 2.3. With notation as in Definition 2.1, suppose that $\omega$ is a holomorphic $r$-form on $\mathbb{C}^{n}$ and let $R>0$ such that $X \cap V(\boldsymbol{f}) \subset B_{R}$. Then $\operatorname{Res}_{X}\left[\begin{array}{c}\omega \\ \boldsymbol{f}^{\boldsymbol{\alpha}+\mathbf{1}}\end{array}\right]$ is equal to

$$
\frac{(-1)^{\frac{r(r-1)}{2}}(|\boldsymbol{\alpha}|+r-1) !}{(2 i \pi)^{r} \boldsymbol{\alpha} !} \int_{\partial\left(X \cap B_{R}\right)}\left(\prod_{j=1}^{r} \bar{f}_{j}^{\alpha_{j}}\right) \frac{\sum_{j=1}^{r}(-1)^{j-1} \bar{f}_{j} \bigwedge_{\substack{l=1 \\ l \neq j}}^{r} \overline{\mathrm{d} f_{l}}}{\left(\sum_{j=1}^{r}\left|f_{j}\right|^{2}\right)^{r+|\boldsymbol{\alpha}|}} \wedge \omega
$$

Proof. When $X=\mathbb{C}^{n}$, such Bochner-Martinelli type integral representation formulae are also known as Andreotti-Norguet formulae, see for instance [BGVY94, §2.4] or TY04, $\$ 3.1$ and 3.2]. We adapt here the proof to the relative case, when $X$ is a $r$-dimensional subvariety in $\mathbb{A}_{\mathbb{C}}^{n}$ with $1 \leq r<n$.

Within this proof, we set for short $\boldsymbol{f}^{\alpha}=\prod_{j=1}^{r} f_{j}^{\alpha_{j}}$. Consider the $(0, r-1)$-form

$$
\Omega_{\boldsymbol{f}, \boldsymbol{\alpha}}=\overline{\boldsymbol{f}}^{\alpha} \sum_{j=1}^{r}(-1)^{j-1} \bar{f}_{j} \bigwedge_{\substack{l=1 \\ l \neq j}}^{r} \overline{\mathrm{d} f_{l}}
$$

on $\mathbb{C}^{n}$, and let $\lambda$ be a formal parameter. A formal computation shows that, if $[X]$ denotes the integration current on $X$, then

$$
\mathrm{d}\left(\frac{\prod_{j=1}^{r}\left|f_{j}\right|^{2 \lambda}}{\|\boldsymbol{f}\|^{2(r+|\boldsymbol{\alpha}|)}} \Omega_{\boldsymbol{f}, \boldsymbol{\alpha}} \wedge \omega \wedge[X]\right)=r \lambda \frac{\left(\prod_{j=1}^{r}\left|f_{j}\right|^{2 \lambda}\right) \boldsymbol{f}^{\boldsymbol{\alpha}}}{\|\boldsymbol{f}\|^{2(r+|\boldsymbol{\alpha}|)}} \bigwedge_{l=1}^{r} \overline{\mathrm{d} f_{l}} \wedge \omega \wedge[X] .
$$

For $\boldsymbol{t} \in\left(\mathbb{R}_{\geq 0}\right)^{n}$, set $\boldsymbol{t}_{\boldsymbol{\alpha}}=\left(t_{1}^{1 /\left(2\left(\alpha_{1}+1\right)\right)}, \ldots, t_{r}^{1 /\left(2\left(\alpha_{r}+1\right)\right)}\right)$ and let $\Gamma_{\boldsymbol{t}_{\boldsymbol{\alpha}}}$ be the associated $r$-dimensional semianalytic chain on $X$ as in 2.1). For $\eta>0$ small enough (depending on $R$ and $\boldsymbol{\alpha})$ and $\boldsymbol{t} \in(0, \eta]^{r}$, we have that $\frac{1}{(2 i \pi)^{r}} \int_{\Gamma_{\boldsymbol{t}_{\boldsymbol{\alpha}}}} \frac{\omega}{f_{1}^{\alpha_{1}+1} \cdots f_{r}^{\alpha_{r}+1}}=\operatorname{Res}_{X}\left[\begin{array}{c}\omega \\ \boldsymbol{f}^{\boldsymbol{\alpha}+\mathbf{1}}\end{array}\right]$. Consider the subset

$$
I(R, \eta)=\left\{\boldsymbol{x} \in X \cap B_{R}|| f_{j}(\boldsymbol{x}) \mid \leq \eta^{\frac{1}{2\left(\alpha_{j}+1\right)}}, j=1, \ldots, r\right\}
$$

and let now $\lambda$ be a complex parameter with $\operatorname{Re}(\lambda) \gg 1$. It follows from Stokes' theorem and from 2.3 that

$$
\begin{aligned}
& \int_{\partial\left(X \cap \partial B_{R}\right)} \frac{\prod_{j=1}^{r}\left|f_{j}\right|^{2 \lambda}}{\|\boldsymbol{f}\|^{2(r+|\boldsymbol{\alpha}|)}} \Omega_{\boldsymbol{f}, \boldsymbol{\alpha}} \wedge \omega \\
& =\int_{\partial I(R, \eta)} \frac{\prod_{j=1}^{r}\left|f_{j}\right|^{2 \lambda}}{\|\boldsymbol{f}\|^{2(r+|\boldsymbol{\alpha}|)}} \Omega_{\boldsymbol{f}, \boldsymbol{\alpha}} \wedge \omega+r \lambda \int_{X \backslash I(R, \eta)} \frac{\left(\prod_{j=1}^{r}\left|f_{j}\right|^{2 \lambda}\right) \boldsymbol{f}^{\boldsymbol{\alpha}}}{\|\boldsymbol{f}\|^{2(r+|\boldsymbol{\alpha}|)}} \bigwedge_{l=1}^{r} \overline{\mathrm{d} f_{l}} \wedge \omega .
\end{aligned}
$$


It follows again from Stokes' theorem and 2.3 that

$$
\begin{aligned}
\int_{\partial I(R, \eta)} \frac{\prod_{j=1}^{r}\left|f_{j}\right|^{2 \lambda}}{\|\boldsymbol{f}\|^{2(r+|\boldsymbol{\alpha}|)}} \Omega_{\boldsymbol{f}, \boldsymbol{\alpha}} \wedge \omega & =r \lambda \int_{I(R, \eta)} \frac{\left(\prod_{j=1}^{r}\left|f_{j}\right|^{2 \lambda}\right) \boldsymbol{f}^{\boldsymbol{\alpha}}}{\|\boldsymbol{f}\|^{2(r+|\boldsymbol{\alpha}|)}} \bigwedge_{l=1}^{r} \overline{\mathrm{d} f_{l}} \wedge \omega \\
& =\frac{r \lambda}{\prod_{l=1}^{r}\left(\alpha_{l}+1\right)} \int_{I(R, \eta)} \frac{\left(\prod_{j=1}^{r}\left|f_{j}\right|^{2 \lambda}\right) \boldsymbol{f}^{\boldsymbol{\alpha}}}{\|\boldsymbol{f}\|^{2(r+|\boldsymbol{\alpha}|)}} \bigwedge_{l=1}^{r} \overline{\mathrm{d} f_{l}^{\alpha_{l}+1}} \wedge \omega .
\end{aligned}
$$

We have that $\bigwedge_{l} \mathrm{~d}\left(\left|f_{l}\right|^{2\left(\alpha_{l}+1\right)}\right)=\boldsymbol{f}^{\boldsymbol{\alpha}+1} \bigwedge_{l}\left(\bar{\partial} f_{l}\right)^{\alpha_{l}+1}$. It follows then from Lebesgue's domination and Fubini's theorems and using the map $\boldsymbol{t}=\left(\left|f_{1}\right|^{2\left(\alpha_{1}+1\right)}, \ldots,\left|f_{r}\right|^{2\left(\alpha_{r}+1\right)}\right)$ to define the slicing locally about each point in $X \backslash W$, that since $W$ has Lebesgue measure 0 with respect to the $r$-dimensional Lebesgue measure on $X$ and the set $\operatorname{Cr}(\boldsymbol{t})$ of critical values of the map $\left.\boldsymbol{t}\right|_{X}$ has Lebesgue measure 0 in $\left(\mathbb{R}_{\geq 0}\right)^{r}$ thanks to Sard's lemma,

$$
\begin{aligned}
& \frac{r \lambda}{\prod_{l=1}^{r}\left(\alpha_{l}+1\right)} \int_{I(R, \eta)} \frac{\left(\prod_{j=1}^{r}\left|f_{j}\right|^{2 \lambda}\right) \boldsymbol{f}^{\boldsymbol{\alpha}}}{\|\boldsymbol{f}\|^{2(r+|\boldsymbol{\alpha}|)}} \bigwedge_{l=1}^{r} \overline{\mathrm{d} f_{l}^{\alpha_{l}+1}} \wedge \omega \\
& =\frac{r \lambda}{\prod_{l=1}^{r}\left(\alpha_{l}+1\right)} \int_{\left\{\boldsymbol{x} \in\left(X \cap B_{R}\right) \backslash W \mid t_{j}(\boldsymbol{x}) \leq \eta, j=1, \ldots, r\right\}} \frac{\prod_{l=1}^{r}\left|t_{l}(\boldsymbol{x})\right|^{\lambda /\left(\alpha_{l}+1\right)}}{\boldsymbol{f}^{\boldsymbol{\alpha}+1}\|\boldsymbol{f}\|^{2(r+|\boldsymbol{\alpha}|)}} \bigwedge_{l=1}^{r} \mathrm{~d} t_{l}(\boldsymbol{x}) \wedge \omega \\
& =(-1)^{(r(r-1) / 2} \frac{r \lambda}{\prod_{l=1}^{r}\left(\alpha_{l}+1\right)} \int_{(0, \eta]^{r} \backslash \operatorname{Cr}(\boldsymbol{t})}\left(\int_{\Gamma_{\boldsymbol{t}_{\boldsymbol{\alpha}}}} \frac{\omega}{\boldsymbol{f}^{\boldsymbol{\alpha}+\mathbf{1}}}\right) \frac{\prod_{l=1}^{r} t_{l}^{\lambda /\left(\alpha_{l}+1\right)}}{\left(\sum_{l=1}^{r} t_{l}^{1 /\left(\alpha_{l}+1\right)}\right)^{r+|\boldsymbol{\alpha}|}} \mathrm{d} \boldsymbol{t} \\
& =(-1)^{(r(r-1) / 2}(2 i \pi)^{r} \operatorname{Res}_{X}\left[\begin{array}{c}
\boldsymbol{\alpha}+\mathbf{1}
\end{array}\right] \times \frac{r \lambda}{\prod_{l=1}^{r}\left(\alpha_{l}+1\right)} \int_{(0, \eta]^{r}} \frac{\prod_{l=1}^{r} t_{l}^{\lambda /\left(\alpha_{l}+1\right)}}{\left(\sum_{l=1}^{r} t_{l}^{1 /\left(\alpha_{l}+1\right)}\right)^{r+|\boldsymbol{\alpha}|}} \mathrm{d} \boldsymbol{t} .
\end{aligned}
$$

We now consider both sides of (2.4) as meromorphic functions of $\lambda$ having no poles in $\operatorname{Re}(\lambda)>-\kappa$ for some sufficiently small value of $\kappa>0$. Identifying the values at $\lambda=0$ of both sides of $(2.4)$, we get

$$
\begin{aligned}
& \int_{\partial\left(X \cap B_{R}\right.} \frac{\Omega_{\boldsymbol{f}, \boldsymbol{\alpha}}}{\|\boldsymbol{f}\|^{2(r+|\boldsymbol{\alpha}|)}} \wedge \omega \\
& =(-1)^{(r(r-1) / 2}(2 i \pi)^{r}\left[\frac{r \lambda}{\prod_{l=1}^{r}\left(\alpha_{l}+1\right)} \int_{(0, \eta]^{r}} \frac{\prod_{l=1}^{r} t_{l}^{\lambda /\left(\alpha_{l}+1\right)}}{\left(\sum_{l=1}^{r} t_{l}^{1 /\left(\alpha_{l}+1\right)}\right)^{r+|\boldsymbol{\alpha}|}} \mathrm{d} \boldsymbol{t}\right]_{\lambda=0} \operatorname{Res}_{X}\left[\begin{array}{c}
\omega \\
\boldsymbol{f}^{\boldsymbol{\alpha}+\mathbf{1}}
\end{array}\right] \\
& =(-1)^{(r(r-1) / 2}(2 i \pi)^{r} \frac{\boldsymbol{\alpha} !}{(|\boldsymbol{\alpha}|+r-1) !} \operatorname{Res}_{X}\left[\begin{array}{c}
\omega \\
\boldsymbol{f}^{\boldsymbol{\alpha}+\mathbf{1}}
\end{array}\right],
\end{aligned}
$$

which leads to 2.3 .

For each subset $I \subset\{1, \ldots, n\}$ of cardinality $r$, write $I=\left\{i_{1}, \ldots, i_{r}\right\}$ with $i_{1}<$ $\cdots<i_{r}$ and consider the holomorphic $r$-form on $\mathbb{C}^{n}$ given by

$$
\mathrm{d} \boldsymbol{x}_{I}=\bigwedge_{j=1}^{r} \mathrm{~d} x_{i_{j}} .
$$

Definition 2.4. A holomorphic $r$-form $\omega$ on $\mathbb{C}^{n}$ is polynomial if it writes down as

$$
\omega=\sum_{I} g_{I} \mathrm{~d} \boldsymbol{x}_{I},
$$

the sum being over the subsets $I \subset\{1, \ldots, n\}$ of cardinality $r$, with $g_{I} \in \mathbb{C}[\boldsymbol{x}]$ for all $I$. It is defined over $\mathbb{Z}$ if $g_{I} \in \mathbb{Z}[\boldsymbol{x}]$ for all $I$. 
A meromorphic $r$-form $\omega$ on $\mathbb{C}^{n}$ is rational if there is $h \in \mathbb{C}[\boldsymbol{x}] \backslash\{0\}$ such that $h \omega$ is a polynomial $r$-form on $\mathbb{C}^{n}$. It is defined over $\mathbb{Q}$ if there is $h \in \mathbb{Z}[\boldsymbol{x}] \backslash\{0\}$ such that $h \omega$ is a polynomial $r$-form defined over $\mathbb{Z}$.

We next list the basic properties of residues on affine varieties. We will restrict to the algebraic setting and in particular, we will only consider polynomial or rational forms, although several of these properties hold in greater generality. As before, we assume that $X \subset \mathbb{A}_{\mathbb{C}}^{n}$ is a variety of pure dimension $r \geq 1$ and that $\boldsymbol{f}=\left(f_{1}, \ldots, f_{r}\right) \in \mathbb{C}[\boldsymbol{x}]^{r}$ is a complete intersection on $X$.

Residues on affine varieties vanish on the ideal generated by $f$ in the ring of regular functions of $X$.

Proposition 2.5. Let $\omega$ be a rational $r$-form that is regular on an open subset $U \subset X$ containing $X \cap V(\boldsymbol{f})$ and $p \in(\boldsymbol{f})$, the ideal generated by $\boldsymbol{f}$ in $\mathcal{O}_{X}(U)$. Then

$$
\operatorname{Res}_{X}\left[\begin{array}{c}
p \omega \\
\boldsymbol{f}
\end{array}\right]=0 .
$$

Proof. See instance [CH78, §4.4, Theorem 4.4.1(2)].

Also, these residues are invariant under linear change of variables.

Proposition 2.6. Let $\ell: \mathbb{A}_{\mathbb{C}}^{n} \mapsto \mathbb{A}_{\mathbb{C}}^{n}$ be an invertible affine map. Then

$$
\operatorname{Res}_{X}\left[\begin{array}{c}
\omega \\
f
\end{array}\right]=\operatorname{Res}_{\ell^{-1}(X)}\left[\begin{array}{l}
\ell^{*} \omega \\
\ell^{*} \boldsymbol{f}
\end{array}\right] .
$$

Proof. See for example [BVY05, page 25].

Another important property is the Lagrange-Jacobi vanishing theorem. Consider the map

$$
\varphi_{\boldsymbol{f}}: X \longmapsto \mathbb{A}_{\mathbb{C}}^{r}, \quad \boldsymbol{x} \longmapsto \boldsymbol{f}(\boldsymbol{x}) .
$$

This map is proper if and only if there exist $\delta_{i}>0, i=1, \ldots, r$, and $C, \tau>0$ such that, for all $\boldsymbol{x} \in X$ with $\|\boldsymbol{x}\| \geq C$,

$$
\sum_{i=1}^{r} \frac{\left|f_{i}(\boldsymbol{x})\right|}{\|\boldsymbol{x}\|^{\delta_{i}}} \geq \tau,
$$

see for instance [Hic01, Theorem 5.2]. In the case when the homogenizations $f_{j}^{\mathrm{h}}$, $j=1, \ldots, r$, have no common zeros in the intersection of the hyperplane at infinity $\left\{x_{0}=0\right\}$ with the Zariski closure of $X$ in $\mathbb{P}_{\mathbb{C}}^{n}$, in the inequality 2.7 we can take

$$
\delta_{i}=\operatorname{deg}\left(f_{i}\right), \quad i=1, \ldots, r .
$$

Theorem 2.7. Suppose that the map $\varphi_{\boldsymbol{f}}$ in $(2.6)$ is proper and let $\boldsymbol{\delta}=\left(\delta_{1}, \ldots, \delta_{r}\right)$ be as in (2.7). Let $\omega$ be a polynomial $r$-form on $\mathbb{C}^{n}$ such that $\operatorname{deg}(\omega)<|\boldsymbol{\delta}|-r$. Then

$$
\operatorname{Res}_{X}\left[\begin{array}{l}
\omega \\
\boldsymbol{f}
\end{array}\right]=0
$$

Proof. See for instance [BVY05, Proposition 4.1].

We also need the next extension of the transformation law for affine residues. 
Theorem 2.8. Let $\boldsymbol{\phi}=\left(\phi_{i}\right)_{1 \leq i \leq r} \in \mathbb{C}[\boldsymbol{x}]^{r}$ and $A=\left(a_{i, j}\right)_{1 \leq i, j \leq r} \in \mathbb{C}[\boldsymbol{x}]^{r \times r}$ such that $\boldsymbol{\phi}$ is a complete intersection on $X$ and $A \cdot \boldsymbol{f}=\boldsymbol{\phi}$. Let $\boldsymbol{u}=\left(u_{1}, \ldots, u_{r}\right)$ be a group of $r$ variables and set $a_{l}=\sum_{i=1}^{r} a_{l, i} u_{i} \in \mathbb{C}[\boldsymbol{u}, \boldsymbol{x}], i=1, \ldots, r$. For $\boldsymbol{\alpha} \in \mathbb{N}^{r}$, set

$$
H=\operatorname{det}(A) \cdot \prod_{l=1}^{r}\left(\sum_{k=0}^{|\boldsymbol{\alpha}|} \phi_{l}^{k} a_{l}^{|\boldsymbol{\alpha}|-k}\right) \in \mathbb{C}[\boldsymbol{u}, \boldsymbol{x}]
$$

and $G=\operatorname{coeff}_{\boldsymbol{u}^{\alpha}}(H) \in \mathbb{C}[\boldsymbol{x}]$, the coefficient of $\boldsymbol{u}^{\boldsymbol{\alpha}}$ in the monomial expansion of $H$ with respect to the group of variables $\boldsymbol{u}$. Let $\omega$ be a polynomial $r$-form on $\mathbb{C}^{n}$. Then

$$
\operatorname{Res}_{X}\left[\begin{array}{c}
\omega \\
\left.f_{1}^{\alpha_{1}+1}, \ldots, f_{r}^{\alpha_{r}+1}\right]
\end{array}\right]=\operatorname{Res}_{X}\left[\begin{array}{c}
G \omega \\
\phi_{1}^{|\boldsymbol{\alpha}|+1}, \ldots, \phi_{r}^{|\boldsymbol{\alpha}|+1}
\end{array}\right] .
$$

Proof. The case when $\boldsymbol{\alpha}=\mathbf{0}$ is done in [BVY05, Proposition 3.2]. The general case when $\boldsymbol{\alpha} \in \mathbb{N}^{r}$ is arbitrary, can be similarly proven by transposing the proof of [BH97] on $\mathbb{C}^{n}$ to the case of an arbitrary affine variety, using the Bochner-Martinelli integral representation of affine residues from Proposition 2.3

When $X$ is the affine line, we can compute the residue of a polynomial 1-form as a coefficient in the Laurent expansion around the point at infinity of a rational function.

Proposition 2.9. Let $f \in \mathbb{C}[x] \backslash\{0\}$ and $\omega=g \mathrm{~d} x$ be a polynomial 1-form on $\mathbb{A}_{\mathbb{C}}^{1}$. Then

$$
\operatorname{Res}_{\mathbb{A}_{\mathbb{C}}^{1}}\left[\begin{array}{l}
\omega \\
f
\end{array}\right]
$$

equals the coefficient of degree -1 in the expansion of $g / f$ as a Laurent series around the point at infinity.

Proof. Consider $\omega / f$ as a rational 1 -form on $\mathbb{P}^{1}(\mathbb{C})$. As usual, we identify the complex plane $\mathbb{C}$ with the open subset $\mathbb{P}^{1}(\mathbb{C}) \backslash\{\infty\}$, with $\infty=(0: 1)$ the point at infinity. With this identification,

$$
\operatorname{Res}_{\mathbb{A}_{\mathbb{C}}}\left[\begin{array}{l}
\omega \\
f
\end{array}\right]=\sum_{\xi \in V(f)} \operatorname{res}_{\xi}\left(\frac{\omega}{f}\right),
$$

where res $\xi$ the action of the local residue at a point $\xi$.

The sum of the local residues of the rational 1-form $\omega / f$ on $\mathbb{P}^{1}(\mathbb{C})$ vanishes, as a consequence of Stokes' theorem on this compact manifold. Hence

$$
\operatorname{Res}_{\mathbb{A}_{\mathbb{C}}^{1}}\left[\begin{array}{l}
\omega \\
f
\end{array}\right]=-\operatorname{res}_{\infty}\left(\frac{\omega}{f}\right)
$$

The local residue is invariant under changes of coordinates. Putting $y=x^{-1}$, we get

$$
\operatorname{res}_{\infty}\left(\frac{\omega}{f}\right)=-\operatorname{res}_{0}\left(\frac{g\left(y^{-1}\right)}{f\left(y^{-1}\right)} \frac{\mathrm{d} y}{y^{2}}\right) .
$$

By Cauchy's integral formula, this coincides with the coefficient of degree 1 in the expansion of $g\left(y^{-1}\right) / f\left(y^{-1}\right)$ as a Laurent series around the origin or, equivalently, with the coefficient of degree -1 in the expansion of $g / f$ as a Laurent series around the point at infinity.

Reciprocally, we can compute the Laurent expansion around the point at infinity of the inverse of a polynomial, in terms of residues over the affine line. 
Corollary 2.10. Let $f \in \mathbb{C}[x] \backslash \mathbb{C}$ and set $d=\operatorname{deg}(f)$. Then, for $x \in \mathbb{C}$ with $|x|>\max _{\xi \in V(f)}|\xi|$,

$$
\frac{1}{f(x)}=\sum_{l \in \mathbb{N}} \operatorname{Res}_{\mathbb{A}_{\mathbb{C}}^{1}}\left[\begin{array}{c}
x^{d+l-1} \mathrm{~d} x \\
f
\end{array}\right] x^{-d-l} .
$$

Proof. We can write $f=\left(f_{d}+x^{-1} q\left(x^{-1}\right)\right) x^{d}$ with $f_{d} \in \mathbb{C}^{\times}$and $q \in \mathbb{C}\left[x^{-1}\right]$. Hence, the Laurent expansion of $1 / f$ around the point at infinity is of the form

$$
\frac{1}{f}=\sum_{l \in \mathbb{N}} c_{l} x^{-d-l}
$$

with $c_{l} \in \mathbb{C}$. Since $1 / f$ is holomorphic for $x \in \mathbb{C}$ with $|x|>\max _{\xi \in V(f)}|\xi|$, this expansion is convergent on this region, and the expression for the $c_{l}$ 's in terms of residues follows from Proposition 2.9 .

2.2. Relationship to traces and division formulae in polynomial rings. Multivariate residue calculus is deeply related to the concept of trace. In particular, traces over reduced 0-dimensional $\mathbb{C}$-algebras can be expressed in terms of residues.

Definition 2.11. Let $K$ be a field, $L$ a finite-dimensional $K$-algebra, and $q \in L$. The trace of $q$, denoted by $\operatorname{Tr}_{L / K}(q)$, is defined as the trace of the multiplication map $m_{g}: L \rightarrow L$ given by $m_{q}(p)=q \cdot p$.

Let $X \subset \mathbb{A}_{\mathbb{C}}^{n}$ be a variety of pure dimension $r \geq 1$ and $\boldsymbol{f}=\left(f_{1}, \ldots, f_{r}\right)$ a complete intersection on $X$. Set

$$
\mathrm{d} \boldsymbol{f}=\bigwedge_{i=1}^{r} \mathrm{~d} f_{i} .
$$

Proposition 2.12. Suppose that the finite-dimensional $\mathbb{C}$-algebra $B=\mathbb{C}[\boldsymbol{x}] /(I(X)+$ $(\boldsymbol{f}))$ is reduced, and let $q \in \mathbb{C}\left(x_{1}, \ldots, x_{n}\right)$ be a rational function that is regular on $X \cap V(\boldsymbol{f})$. Then

$$
\operatorname{Tr}_{B / \mathbb{C}}(q)=\operatorname{Res}_{X}\left[\begin{array}{c}
q \mathrm{~d} \boldsymbol{f} \\
\boldsymbol{f}
\end{array}\right]
$$

Proof. Write $X \cap V(\boldsymbol{f})=\left\{\boldsymbol{\xi}_{1}, \ldots, \boldsymbol{\xi}_{L}\right\}$ with $L=\#(X \cap V(\boldsymbol{f}))$ and $\boldsymbol{\xi}_{l} \in \mathbb{C}^{n}$. Since $B$ is reduced, the map

$$
\psi: B \longrightarrow \mathbb{C}^{L}, \quad q \longmapsto\left(q\left(\boldsymbol{\xi}_{1}\right), \ldots, q\left(\boldsymbol{\xi}_{L}\right)\right)
$$

is an isomorphism of $\mathbb{C}$-algebras. For $q \in B$, the matrix of the multiplication map $m_{q}$ in the standard basis $\mathcal{S}$ of $\mathbb{C}^{L}$ is diagonal, namely $\left(m_{q}\right)_{\mathcal{S}}=\operatorname{diag}\left(q\left(\boldsymbol{\xi}_{1}\right), \ldots, q\left(\boldsymbol{\xi}_{L}\right)\right) \in$ $\mathbb{C}^{L \times L}$. Hence

$$
\operatorname{Tr}_{B / \mathbb{C}}(q)=\sum_{l=1}^{L} q\left(\boldsymbol{\xi}_{l}\right) .
$$

Set $Z(\boldsymbol{f})=X \cdot \prod_{j=1}^{r} \operatorname{div}\left(f_{j}\right)$ for the 0-dimensional intersection cycle of $\boldsymbol{f}$ on $X$, and let $[Z(\boldsymbol{f})]$ be the integration current on this cycle. By [CH78, $\S 1.9$ and 3.6], we have the currential identity

$$
\left(\bigwedge_{j=1}^{r} \bar{\partial}\left(\frac{1}{f_{j}}\right) \wedge X\right) \wedge \mathrm{d} \boldsymbol{f}=[Z(\boldsymbol{f})]
$$


Since $B$ is reduced, so is $Z(\boldsymbol{f})$ and hence $[Z(\boldsymbol{f})]=\sum_{l=1}^{L} \delta_{\boldsymbol{\xi}_{l}}$, where $\delta_{\boldsymbol{\xi}_{l}}$ denotes the Dirac delta measure at the point $\boldsymbol{\xi}_{l}$. By $(2.2)$,

$$
\operatorname{Res}_{X}\left[\begin{array}{c}
q \mathrm{~d} \boldsymbol{f} \\
\boldsymbol{f}
\end{array}\right]=\left\langle\bigwedge_{j=1}^{r} \bar{\partial}\left(\frac{1}{f_{j}}\right) \wedge[X], \chi q \mathrm{~d} \boldsymbol{f}\right\rangle=\int_{X} \chi q \mathrm{~d}[Z(\boldsymbol{f})]=\sum_{l=1}^{L} q\left(\boldsymbol{\xi}_{l}\right),
$$

where $\chi: \mathbb{C}^{n} \rightarrow \mathbb{R}$ is an arbitrary $C^{\infty}$-function with compact support that is identically equal to 1 on a neighborhood of $X \cap V(\boldsymbol{f})$. The statement follows from this equality together with 2.11).

We can also consider traces of rational functions on $X$, which are rational functions over the base space $\mathbb{C}^{r}$. The hypothesis that $\boldsymbol{f}$ is complete intersection over $X$ implies that the map $\varphi_{f}$ in 2.6$)$ is dominant and generically finite. Let $K=\mathrm{K}\left(\mathbb{A}_{\mathbb{C}}^{r}\right)$ and $L=\mathrm{K}(X)$ respectively denote the function fields of $\mathbb{A}_{\mathbb{C}}^{r}$ and of $X$, and let $\varphi_{f}^{\#}: K \hookrightarrow L$ be the finite field extension induced by this map. We identify $K$ with the field $\mathbb{C}(\boldsymbol{y})$, where $\boldsymbol{y}=\left(y_{1}, \ldots, y_{r}\right)$ denotes a group of $r$ variables.

Let $g, h \in \mathbb{C}[\boldsymbol{x}]$ such that $h \notin I(X)$. Then $q=g / h$ is a rational function on $X$, and the trace

$$
\Theta_{X, \boldsymbol{f}, q}:=\operatorname{Tr}_{L / K}(q) \in K=\mathbb{C}(\boldsymbol{y})
$$

is a rational function on $\mathbb{C}^{r}$. Under suitable hypothesis, the Taylor expansion of this rational function can be computed in terms of affine residues. Set

$$
D=\left(\prod_{i=1}^{r} d_{i}\right) \operatorname{deg}(X)
$$

for the Bézout number of $\boldsymbol{f}$ on $X$.

Proposition 2.13. Suppose that $\#(X \cap V(\boldsymbol{f}))=D$ and let $g \in \mathbb{C}[\boldsymbol{x}]$. Then $\Theta_{X, \boldsymbol{f}, g} \in$ $\mathbb{C}[\boldsymbol{y}]$ and

$$
\Theta_{X, \boldsymbol{f}, g}=\sum_{\boldsymbol{\alpha}} \operatorname{Res}_{X}\left[\begin{array}{l}
g \mathrm{~d} \boldsymbol{f} \\
\boldsymbol{f}^{\boldsymbol{\alpha}+\mathbf{1}}
\end{array}\right] \boldsymbol{y}^{\boldsymbol{\alpha}}
$$

the sum being over the vectors $\boldsymbol{\alpha} \in \mathbb{N}^{r}$ such that $\sum_{j=1}^{r} \alpha_{j} \operatorname{deg}\left(f_{j}\right) \leq \operatorname{deg}(g)$.

We give the proof of this result after the next lemma. This lemma shows that, on a nonempty open subset of $\mathbb{C}^{r}$, the function $\Theta_{X, \boldsymbol{f}, g}$ can be computed in terms of traces over "fiber" algebras.

Lemma 2.14. Let notation be as in Proposition 2.13 and set, for $\boldsymbol{y} \in \mathbb{C}^{r}$,

$$
B_{\boldsymbol{y}}=\mathbb{C}[\boldsymbol{x}] /(I(X)+(\boldsymbol{f}-\boldsymbol{y})) .
$$

Then there is nonempty open subset $U \subset \mathbb{C}^{r}$ such that, for $\boldsymbol{y} \in W$, the $\mathbb{C}$-algebra $B_{\boldsymbol{y}}$ is reduced, $\operatorname{dim}_{\mathbb{C}}\left(B_{\boldsymbol{y}}\right)=D$, and

$$
\Theta_{X, \boldsymbol{f}, q}(\boldsymbol{y})=\operatorname{Tr}_{B_{\boldsymbol{y}} / \mathbb{C}}(q) .
$$

Proof. The hypothesis that $\#(X \cap V(\boldsymbol{f}))=D$ is equivalent to the fact that the fiber of $\varphi_{\boldsymbol{f}}$ at the point $\mathbf{0} \in \mathbb{C}^{r}$ has exactly $D$ points. By Bézout's theorem, this fiber is reduced and moreover, there is a nonempty open subset $U_{1} \subset \mathbb{C}^{r}$ with $\mathbf{0} \in U_{1}$ such that, for $\boldsymbol{y} \in U_{1}$,

$$
\# \varphi_{\boldsymbol{f}}^{-1}(\boldsymbol{y})=\#(X \cap V(\boldsymbol{f}-\boldsymbol{y}))=D
$$

and $\varphi_{\boldsymbol{f}}^{-1}(\boldsymbol{y})$ is also reduced.

For the third statement, let $\ell \in \mathbb{C}[\boldsymbol{x}]$ be a linear form such that $\ell(\xi) \neq \ell\left(\xi^{\prime}\right)$ for all $\xi, \xi^{\prime} \in \varphi_{\boldsymbol{f}}^{-1}(\mathbf{0})$ with $\xi \neq \xi^{\prime}$. Then $\mathcal{B}=\left(\ell^{k}\right)_{0 \leq k \leq D-1}$ gives a basis for the $\mathbb{C}$-algebra $B_{\mathbf{0}}$. 
Let $U_{2} \subset U_{1}$ be nonempty open subset $\mathbf{0} \in U_{2}$ where the fibers of $\varphi_{\boldsymbol{f}}$ have cardinality $D$ and the linear form $\ell$ separates the point of these fibers. Similarly, the collection $\mathcal{B}$ is a $\mathbb{C}$-basis of the fiber algebra $B_{\boldsymbol{y}}$ for all $\boldsymbol{y} \in U_{2}$. In particular, this also implies that $\mathcal{B}$ is a $K$-basis of the $K$-algebra $L$.

For $0 \leq j, k \leq D-1$, write

$$
\ell^{j} \cdot \ell^{k}=\sum_{l} \gamma_{j, k, l} \ell^{l}
$$

with $\gamma_{j, k, l} \in K$. Then we choose $U$ as any nonempty open subset of $U_{2}$ such that $\mathbf{0} \in U$ and where all the rational functions $\gamma_{j, k, l}$ are regular. Hence, the relations in (2.13) also hold in the $\mathbb{C}$-algebra $B_{\boldsymbol{y}}$, for $\boldsymbol{y} \in U$.

Let $M_{q}=\left(m_{q}\right)_{\mathcal{B}} \in K^{D \times D}$ be the matrix of the multiplication map of $q$ over $L$ with respect to this basis. For $\boldsymbol{y} \in U$, this matrix specializes into the matrix of the multiplication map of $q$ over $B_{\boldsymbol{y}}$ with respect to the basis $\mathcal{B}$. Hence

$$
\Theta_{X, \boldsymbol{f}, q}(\boldsymbol{y})=\operatorname{Tr}\left(M_{q}(\boldsymbol{y})\right)=\operatorname{Tr}_{B_{\boldsymbol{y}} / \mathbb{C}}(q),
$$

as stated.

Proof of Proposition 2.13. By Lemma 2.14 and Proposition 2.12, there is a nonempty open subset $U \subset \mathbb{C}^{r}$ with $\mathbf{0} \in U$ such that, for $\boldsymbol{y} \in U$,

$$
\Theta_{X, \boldsymbol{f}, g}(\boldsymbol{y})=\operatorname{Res}_{X}\left[\begin{array}{c}
g \mathrm{~d} \boldsymbol{f} \\
\boldsymbol{f}-\boldsymbol{y}
\end{array}\right] .
$$

Hence, the rational function $\Theta_{X, \boldsymbol{f}, g}$ is regular at $\mathbf{0} \in \mathbb{C}^{r}$, and we can consider its Taylor expansion around this point.

Since the set-valued function $\boldsymbol{y} \mapsto X \cap V(\boldsymbol{f}-\boldsymbol{y})$ varies continuously on a neighborhood of $\mathbf{0} \in \mathbb{C}^{r}$, there exist $R>0$ and $\eta>0$ such that $X \cap V(\boldsymbol{f}-\boldsymbol{y}) \subset X \cap B_{R}$ for all $\boldsymbol{y} \in \mathbb{C}^{r}$ with $\|\boldsymbol{y}\|<\eta$. By Proposition 2.3.

$$
\operatorname{Res}_{X}\left[\begin{array}{c}
g \mathrm{~d} \boldsymbol{f} \\
\boldsymbol{f}-\boldsymbol{y}
\end{array}\right]=\frac{(-1)^{r(r-1) / 2}(r-1) !}{(2 i \pi)^{r}} \int_{\partial\left(X \cap B_{R}\right)} \frac{\Omega_{\boldsymbol{f}-\boldsymbol{y}}}{\|\boldsymbol{f}-\boldsymbol{y}\|^{2 r}} \wedge g \mathrm{~d} \boldsymbol{f}
$$

with $\Omega_{\boldsymbol{f}-\boldsymbol{y}}=\sum_{j=1}^{r}(-1)^{j-1}\left(\overline{f_{j}}-\overline{y_{j}}\right) \bigwedge_{\substack{l=1 \\ l \neq j}}^{r} \overline{\mathrm{d} f_{l}}$. Differentiating this identity, it follows from Lebesgue's differentiation theorem that

$$
\frac{\partial^{|\boldsymbol{\alpha}|} \Theta_{X, \boldsymbol{f}, g}}{\partial \boldsymbol{y}^{\boldsymbol{\alpha}}}=\frac{(-1)^{(r(r-1) / 2}(r+|\boldsymbol{\alpha}|-1) !}{(2 i \pi)^{r}} \int_{\partial\left(X \cap B_{R}\right)}(\overline{\boldsymbol{f}}-\overline{\boldsymbol{y}})^{\boldsymbol{\alpha}} \frac{\Omega_{\boldsymbol{f}-\boldsymbol{y}}}{\|\boldsymbol{f}\|^{2(r+|\boldsymbol{\alpha}|)}} \wedge g \mathrm{~d} \boldsymbol{f} .
$$

Evaluating this identity at $\boldsymbol{y}=\mathbf{0}$, we get from the integral representation in Proposition 2.3 that

$$
\frac{\partial^{|\boldsymbol{\alpha}|} \Theta_{X, \boldsymbol{f}, g}}{\partial \boldsymbol{y}^{\boldsymbol{\alpha}}}(\mathbf{0})=\boldsymbol{\alpha} ! \operatorname{Res}_{X}\left[\begin{array}{l}
g \mathrm{~d} \boldsymbol{f} \\
\boldsymbol{f}^{\boldsymbol{\alpha}+\mathbf{1}}
\end{array}\right] .
$$

The hypothesis that $\#(X \cap V(\boldsymbol{f}))=D$ implies that the system $\boldsymbol{f}$ on $X$ has no zeros at infinity. By (2.8) and the Lagrange-Jacobi theorem 2.7, the residues in (2.14) vanish for $\boldsymbol{\alpha} \in \mathbb{N}^{r}$ such that

$$
\operatorname{deg}(g \mathrm{~d} \boldsymbol{f})<\left(\sum_{j=1}^{r}\left(\alpha_{j}+1\right) \operatorname{deg}\left(f_{j}\right)\right)-r .
$$

We have that $\operatorname{deg}(g \mathrm{~d} \boldsymbol{f})=\operatorname{deg}(g)+\left(\sum_{j=1}^{r} \operatorname{deg}\left(f_{j}\right)\right)-r$. Hence, the residues in 2.14 vanish whenever $\sum_{j=1}^{r} \alpha_{j} \operatorname{deg}\left(f_{j}\right)>\operatorname{deg}(g)$, which finishes the proof. 
Residues also play an important role in division formulae in polynomial rings. An example of this connection is the Bergman-Weil trace formula for the case when $X=$ $\mathbb{A}_{\mathbb{C}}^{n}$, see [AY83, II §9] and [Tsi92, IV], or [BH99] for an extended bibliography on this subject as well as a presentation of Weil's formula and Bergman-Weil's developments within an algebraic setting.

To describe this formula, let $\boldsymbol{z}=\left(z_{1}, \ldots, z_{n}\right)$ be a group of variables, fix $1 \leq i \leq n$, let $h_{i, j} \in \mathbb{C}[\boldsymbol{x}, \boldsymbol{z}], 1 \leq j \leq n$, be a family of $n$ polynomials such that

$$
f_{i}(\boldsymbol{z})-f_{i}(\boldsymbol{x})=\sum_{j=1}^{n} h_{i, j}(\boldsymbol{x}, \boldsymbol{z})\left(z_{k}-x_{k}\right),
$$

and set $h_{i}=\sum_{j=1}^{n} h_{i, j} \mathrm{~d} z_{j}$. This is a polynomial 1-form in the variables $\boldsymbol{z}$ whose coefficients are polynomials in $\mathbb{C}[\boldsymbol{x}]$.

Theorem 2.15. With notation as above, let $p \in \mathbb{C}[\boldsymbol{x}]$ and choose $R>0$ such that $X \cap V(f)$ is contained in the ball $B_{R}$. Then, for $\boldsymbol{x} \in \mathbb{C}^{n}$ such that $\|\boldsymbol{f}(\boldsymbol{x})\|<$ $\min _{\boldsymbol{y} \in X \cap \partial B_{R}}\|\boldsymbol{f}(\boldsymbol{y})\|$,

$$
p(\boldsymbol{x})=\sum_{\boldsymbol{\alpha} \in \mathbb{N}^{n}} \operatorname{Res}_{\mathbb{A}_{\mathbb{C}}^{n}}\left[\begin{array}{c}
p(\boldsymbol{z}) \bigwedge_{i=1}^{n} h_{i}(\boldsymbol{x}, \boldsymbol{z}) \\
f_{1}(\boldsymbol{z})^{\alpha_{1}+1}, \ldots, f_{n}(\boldsymbol{z})^{\alpha_{n}+1}
\end{array}\right] f(\boldsymbol{x})^{\boldsymbol{\alpha}} .
$$

When the map $\varphi_{\boldsymbol{f}}$ in $(2.6)$ is proper, the Lagrange-Jacobi theorem 2.7 implies that all but a finite number of residues in the expansion (2.16) vanish. Hence in the proper case, this expansion becomes a polynomial identity.

As an application of the Bergman-Weil formula, we can express the coefficients of the $f$-adic expansion of a univariate polynomial in terms of residues on the affine line.

Definition 2.16. Given $f \in \mathbb{C}[x] \backslash \mathbb{C}$, the $f$-adic expansion of a polynomial $p \in \mathbb{C}[x]$ is its unique finite representation as

$$
p=\sum_{\alpha \in \mathbb{N}} p_{f, \alpha} f^{\alpha}
$$

with $p_{f, \alpha} \in \mathbb{C}[x]$ satisfying $\operatorname{deg}\left(p_{f, \alpha}\right) \leq \operatorname{deg}(f)-1$ for all $\alpha$.

Corollary 2.17. With notation as in Definition 2.16, coefficients of the $f$-adic expression of $p$ are given, for $\alpha \in \mathbb{N}$, by

$$
p_{f, \alpha}(x)=\operatorname{Res}_{\mathbb{A}_{\mathbb{C}}^{1}}\left[p(z) \frac{f(z)-f(x)}{z-x} \mathrm{~d} z\right] .
$$

Proof. For $n=1$, the formula in Theorem 2.15 reduces to

$$
p(x)=\sum_{\alpha \in \mathbb{N}} \operatorname{Res}_{\mathbb{A}_{\mathbb{C}}^{1}}\left[p(z) \frac{f(z)-f(x)}{z-x} \mathrm{~d} y\right] f(x)^{\alpha} .
$$

Set $d=\operatorname{deg}(f)$. The quotient $(f(z)-f(x)) /(z-x)$ is a polynomial in $\mathbb{C}[x, y]$ of degree bounded by $d-1$, and so the residues in the right-hand side of (2.17) are also polynomials in $\mathbb{C}[x]$ of degree bounded by $d-1$.

By the Lagrange-Jacobi theorem 2.7, these residues vanish when $\operatorname{deg}(p)+d-1<$ $(\alpha+1) d-1$ or, equivalently, when $\operatorname{deg}(p)<\alpha d$. Hence, the representation 2.18) is finite, and so it gives the $f$-adic expansion of $p$, as stated. 


\section{Residues on the AfFine Line}

Here we consider the problem of bounding the residues of polynomial and rational 1 -forms on the affine line. More precisely, let $f \in \mathbb{Z}[x] \backslash \mathbb{Z}$ and $g, h \in \mathbb{Z}[x]$ with $h$ coprime with $f$. For $\alpha \in \mathbb{N}$, the residue

$$
\operatorname{Res}_{\mathbb{A}_{\mathbb{C}}^{1}}\left[\begin{array}{c}
g / h \mathrm{~d} x \\
f^{\alpha+1}
\end{array}\right]
$$

is a rational number, and we want to bound its numerator and denominator. In this section, we only consider residues of this type and, for ease of notation, we omit the variety $\mathbb{A}_{\mathbb{C}}^{1}$ when denoting them.

Definition 3.1. Let $f=\sum_{i=0}^{d} f_{i} x^{i} \in \mathbb{Z}[x]$. The (logarithmic) height and the (logarithmic) length of $f$ are respectively defined as

$$
\mathrm{h}(f)=\log \left(\max _{0 \leq i \leq d}\left|f_{i}\right|\right) \quad \text { and } \quad \mathrm{h}_{1}(f)=\log \left(\sum_{i=0}^{d}\left|f_{i}\right|\right) .
$$

These quantities are related by the inequalities

$$
\mathrm{h}(f) \leq \mathrm{h}_{1}(f) \leq \mathrm{h}(f)+\log (d+1) .
$$

The length is submultiplicative, in the sense that, for $f_{1}, f_{2} \in \mathbb{Z}[x]$,

$$
\mathrm{h}_{1}\left(f_{1} f_{2}\right) \leq \mathrm{h}_{1}\left(f_{1}\right)+\mathrm{h}_{1}\left(f_{2}\right) \text {. }
$$

The following is the main result of this section. It bounds the numerators and denominators of the residue sequence of to a polynomial 1-form on the affine line.

Theorem 3.2. Let $f \in \mathbb{Z}[x] \backslash \mathbb{Z}$ and $g \in \mathbb{Z}[x]$. Set $d=\operatorname{deg}(f)$ and $e=\operatorname{deg}(g)$, and let $f_{d}$ be the leading coefficient of $f$. Then, for $\alpha \in \mathbb{N}$,

$$
f_{d}^{e+1-(\alpha+1)(d-1)} \operatorname{Res}\left[\begin{array}{l}
g \mathrm{~d} x \\
f^{\alpha+1}
\end{array}\right] \in \mathbb{Z}
$$

and

$\log \left|f_{d}^{e+1-(\alpha+1)(d-1)} \operatorname{Res}\left[\begin{array}{l}g \mathrm{~d} x \\ f^{\alpha+1}\end{array}\right]\right| \leq \mathrm{h}_{1}(g)+(e+1-(\alpha+1) d) \mathrm{h}(f)+(e-d+1) \log (2)$.

If $e<(\alpha+1) d-1$, then $\operatorname{Res}\left[\begin{array}{l}g \mathrm{~d} x \\ f^{\alpha+1}\end{array}\right]=0$.

We give the proof of this theorem after some preliminary results. Let $f \in \mathbb{C}[x] \backslash \mathbb{C}$ and, for $j, \alpha \in \mathbb{N}$, set

$$
\varrho_{f}(j, \alpha)=\operatorname{Res}\left[\begin{array}{c}
x^{j} \mathrm{~d} x \\
f^{\alpha+1}
\end{array}\right] .
$$

Set also $\varrho_{f}(j,-1)=0$ for all $j \in \mathbb{N}$.

Proposition 3.3. With notation as in (3.1), write $f=\sum_{i=0}^{d} f_{i} x^{i}$ with $d=\operatorname{deg}(f)$ and $f_{i} \in \mathbb{C}$. Then, for all $j, \alpha \in \mathbb{N}$,

$$
\sum_{i=0}^{d} f_{i} \varrho(j+i, \alpha)=\varrho(j, \alpha-1) .
$$

Proof. By linearity of the residue, the sum in the left-hand side of $(3.2)$ is equal to $\operatorname{Res}\left[\begin{array}{c}f x^{j} \mathrm{~d} x \\ f^{\alpha+1}\end{array}\right]$. The identity Res $\left[\begin{array}{c}f x^{j} \mathrm{~d} x \\ f^{\alpha+1}\end{array}\right]=\varrho(j, \alpha-1)$ follows easily from the definition of the residue. 
Using the formula in the previous proposition, we obtain a recursive algorithm for computing the residue sequence of a monomial 1-form.

Proposition 3.4. Let notation be as in Proposition 3.3. Then, for all $j, \alpha \in \mathbb{N}$,

$$
\varrho_{f}(j, \alpha)= \begin{cases}0 & \text { if } j \leq(\alpha+1) d-2, \\ f_{d}^{-\alpha-1} & \text { if } j=(\alpha+1) d-1, \\ \varrho_{f}(j-d, \alpha-1)-\sum_{i=1}^{d} f_{d}^{-1} f_{d-i} \varrho_{f}(j-i, \alpha) & \text { if } j \geq(\alpha+1) d .\end{cases}
$$

Proof. By Proposition 2.9, the residue $\varrho_{f}(j, \alpha)$ coincides with the coefficient of degree -1 in the expansion of $x^{j} / f^{\alpha+1}$ as a Laurent series around the point at infinity. Write $f=\left(f_{d}+x^{-1} q\right) x^{d}$ with $f_{d} \in \mathbb{C}^{\times}$and $q \in \mathbb{C}\left[x^{-1}\right]$. Hence

$$
\frac{x^{j}}{f^{\alpha+1}}=f_{d}^{-\alpha-1} x^{j-(\alpha+1) d}+\text { higher order terms, }
$$

which implies first and second equalities in the statement. The third equality follows from Proposition 3.3

When $f$ has integral coefficients, we can apply the recursive formulae in Proposition 3.4 to bound the numerator and the denominator in the residue sequence of a monomial 1-form.

Proposition 3.5. With notation as in (3.1), suppose that $f \in \mathbb{Z}[x] \backslash \mathbb{Z}$, and denote by $d$ and $f_{d}$ its degree and leading coefficient. Then, for all $j, \alpha \in \mathbb{N}$,

$$
f_{d}^{j+1-(\alpha+1)(d-1)} \varrho_{f}(j, \alpha) \in \mathbb{Z}
$$

and

$$
\log \left|f_{d}^{j+1-(\alpha+1)(d-1)} \varrho_{f}(j, \alpha)\right| \leq(j+1-(\alpha+1) d) \mathrm{h}(f)+(j-d+1) \log (2) .
$$

If $j<(\alpha+1) d-1$, then $\varrho_{f}(j, \alpha)=0$.

Proof. Set $\widetilde{\varrho}_{f}(j, \alpha)=f_{d}^{j+1-(\alpha+1)(d-1)} \varrho_{f}(j, \alpha)$. The recursive formulae in Proposition 3.4 then translate into the relations, for $j, \alpha \in \mathbb{N}$,

$$
\widetilde{\varrho}_{f}(j, \alpha)= \begin{cases}0 & \text { if } j \leq(\alpha+1) d-2, \\ 1 & \text { if } j=(\alpha+1) d-1, \\ \widetilde{\varrho}_{f}(j-d, \alpha-1)-\sum_{i=1}^{d} f_{d}^{i-1} f_{d-i} \widetilde{\varrho}_{f}(j-i, \alpha) & \text { if } j \geq(\alpha+1) d .\end{cases}
$$

Set $H=\mathrm{e}^{\mathrm{h}(f)}=\max _{i}\left|f_{i}\right|$. The statements (3.3) and (3.4) amount to the conditions

$$
\widetilde{\varrho}_{f}(j, \alpha) \in \mathbb{Z} \quad \text { and } \quad\left|\widetilde{\varrho}_{f}(j, \alpha)\right| \leq 2^{j-d+1} H^{j+1-(\alpha+1) d} .
$$

We prove them by induction on the quantity $j+\alpha$. If $j+\alpha=0$, then $j=\alpha=0$ and both statements follow from the recursive formulae above.

Suppose that $j+\alpha \geq 1$. If $j \leq(\alpha+1) d-1$, this also follows from these formulae. So we assume that $j \geq(\alpha+1) d$. By the inductive hypothesis, $\widetilde{\varrho}_{f}(j, \alpha) \in \mathbb{Z}$, which gives (3.3). For the bound (3.4), we use again the inductive hypothesis and these 
formulae to obtain that

$$
\begin{aligned}
\left|\widetilde{\varrho}_{f}(j, \alpha)\right| & \leq\left|\widetilde{\varrho}_{f}(j-d, \alpha-1)\right|+\sum_{i=1}^{d} H^{i}\left|\widetilde{\varrho}_{f}(j-i, \alpha)\right| \\
& \leq 2^{j-2 d+1} H^{j-d+1-\alpha d}+\sum_{i=1}^{d} H^{i} 2^{(j-i)-d+1} H^{(j-i)+1-(\alpha+1) d} \\
& =\left(2^{j-2 d+1}+\sum_{i=1}^{d} 2^{(j-i)-d+1}\right) H^{j+1-(\alpha+1) d} \\
& =2^{j-d+1} H^{j+1-(\alpha+1) d} .
\end{aligned}
$$

The last statement follows directly from Proposition 3.4 or, alternatively, it can be derived from the Lagrange-Jacobi vanishing theorem 2.7 .

Proof of Theorem 3.2. Write $g=\sum_{j=0}^{e} g_{j} x^{j}$ with $g_{j} \in \mathbb{Z}$. By linearity,

$$
\operatorname{Res}\left[\begin{array}{l}
g \mathrm{~d} x \\
f^{\alpha+1}
\end{array}\right]=\sum_{j=0}^{e} g_{j} \operatorname{Res}\left[\begin{array}{c}
x^{j} \mathrm{~d} x \\
f^{\alpha+1}
\end{array}\right] .
$$

Hence, with the notation in the proof of Proposition 3.5 .

$$
f_{d}^{e+1-(\alpha+1)(d-1)} \operatorname{Res}\left[\begin{array}{l}
g \mathrm{~d} x \\
f^{\alpha+1}
\end{array}\right]=\sum_{j=0}^{e} f_{d}^{e-j} g_{j} \widetilde{\varrho}_{f}(j, \alpha) .
$$

It follows from this proposition that this quantity lies in $\mathbb{Z}$, proving the first statement. By the same result,

$$
\begin{aligned}
&\left|\sum_{j=0}^{e} f_{d}^{e-j} g_{j} \widetilde{\varrho}_{f}(j, \alpha)\right| \leq\left(\sum_{j=0}^{e}\left|g_{j}\right|\right) \max _{j}\left(\left|f_{d}\right|^{e-j} 2^{j-d+1} H^{j+1-(\alpha+1) d}\right) \\
& \leq\left(\sum_{j=0}^{e}\left|g_{j}\right|\right) 2^{e-d+1} H^{e+1-(\alpha+1) d}
\end{aligned}
$$

with $H=\mathrm{e}^{\mathrm{h}(f)}=\max _{i}\left|f_{i}\right|$. The second statement follows from this and (3.5). The last statement follows from (3.5) and Proposition 3.5 .

The bounds in Theorem 3.2 are essentially optimal, as shown by the next example.

Example 3.6. Let $d \geq 1, \alpha \geq 0, e \geq(\alpha+1) d, H_{2} \geq H_{1} \geq 1$ and $H_{3} \geq 1$. Set

$$
f=H_{1} x^{d}-H_{2} x^{d-1}, \quad g=H_{3} x^{e} \quad \text { and } \quad \rho=\operatorname{Res}\left[\begin{array}{l}
g \mathrm{~d} x \\
f^{\alpha+1}
\end{array}\right] .
$$

We have that

$$
\rho=\operatorname{Res}\left[\begin{array}{c}
H_{3} x^{e} \mathrm{~d} x \\
\left(x^{d-1}\left(H_{1} x-H_{2}\right)\right)^{\alpha+1}
\end{array}\right]=H_{3} \operatorname{Res}\left[\begin{array}{c}
x^{e-(\alpha+1)(d-1)} \mathrm{d} x \\
H_{1} x-H_{2}
\end{array}\right] .
$$


Making the change of variables $y=H_{1} x-H_{2}$ and applying Propositions 2.6 and 2.9. we get

$$
\begin{aligned}
\rho=H_{3} \operatorname{Res}\left[\begin{array}{c}
\left(H_{1}^{-1}\left(y+H_{2}\right)\right)^{e-(\alpha+1)(d-1)} H_{1}^{-1} \mathrm{~d} y \\
y^{\alpha+1}
\end{array}\right. & \\
& =\left(\begin{array}{c}
e-(\alpha+1) d \\
\alpha
\end{array}\right) \frac{H_{3} H_{2}^{e+1-(\alpha+1) d}}{H_{1}^{e+1-(\alpha+1)(d-1)}} .
\end{aligned}
$$

We have that $\operatorname{deg}(f)=d, \mathrm{~h}(f)=\log \left(H_{2}\right), \operatorname{deg}(g)=e$, and $\mathrm{h}_{1}(g)=\log \left(H_{3}\right)$. In this case, Theorem 3.2 says that

$$
H_{1}^{e+1-(\alpha+1)(d-1)} \rho \in \mathbb{Z} \quad \text { and } \quad\left|H_{1}^{e+1-(\alpha+1)(d-1)} \rho\right| \leq 2^{e-d+1} H_{3} H_{2}^{e+1-(\alpha+1) d},
$$

which can be compared with the explicit expression for $\rho$ in (3.6).

As an application of these results, we derive a bound for the coefficients of the Laurent expansion around the point at infinity of the inverse of a polynomial. For a polynomial $f \in \mathbb{Z}[x] \backslash \mathbb{Z}$ of degree $d$ and $\alpha \in \mathbb{N}$, by Corollary 2.10 the Laurent series

$$
f^{-\alpha-1}=\sum_{l \in \mathbb{N}} c_{f, \alpha, l} x^{-(\alpha+1) d-l}
$$

is convergent when $|x|>\max _{\xi \in V(f)}|\xi|$, and its coefficients can be expressed in terms of residues on the affine line as

$$
c_{f, \alpha, l}=\operatorname{Res}\left[\begin{array}{c}
x^{(\alpha+1) d+l-1} \mathrm{~d} x \\
f^{\alpha+1}
\end{array}\right] .
$$

Corollary 3.7. Let $f \in \mathbb{Z}[x] \backslash \mathbb{Z}$ and $\alpha \in \mathbb{N}$. Set $d=\operatorname{deg}(f)$ and let $f_{d}$ be the leading coefficient of $f$. Then the coefficients of the Laurent expansion of $f^{-\alpha-1}$ in (3.7) satisfy

$$
f_{d}^{l+\alpha+1} c_{f, \alpha, l} \in \mathbb{Z} \quad \text { and } \quad \log \left|f_{d}^{l+\alpha+1} c_{f, \alpha, l}\right| \leq l \mathrm{~h}(f)+(l+\alpha d) \log (2) .
$$

Proof. This follows directly from the formula 3.8 and Proposition 3.5 .

As a second application, we bound the coefficients of the $f$-adic expansion of a polynomial (Definition 2.16).

Proposition 3.8. Let $f \in \mathbb{Z}[x] \backslash \mathbb{Z}$ and $p \in \mathbb{Z}[x]$. Set $d=\operatorname{deg}(f)$ and $e=\operatorname{deg}(p)$, and let $f_{d}$ be the leading coefficient of $f$. Then

$$
p=\sum_{\alpha=0}^{\lfloor e / d\rfloor} p_{f, \alpha} f^{\alpha}
$$

with $p_{f, \alpha} \in \mathbb{Q}[x]$ such that $\operatorname{deg}\left(p_{f, \alpha}\right) \leq d-1$ and

$f_{d}^{e+1-\alpha(d-1)} p_{f, \alpha} \in \mathbb{Z}[x] \quad$ and $\quad \mathrm{h}_{1}\left(f_{d}^{e+1-\alpha(d-1)} p_{f, \alpha}\right) \leq \mathrm{h}_{1}(p)+(e-\alpha d) \mathrm{h}_{1}(f)+e \log (2)$, for all $\alpha$.

Proof. For $\alpha \in \mathbb{N}$, let $p_{f, \alpha} \in \mathbb{Q}[x]$ be the $\alpha$-th coefficient in the $f$-adic expansion of $p$. Clearly, $p_{f, \alpha}=0$ for $\alpha>e / d$.

For $0 \leq \alpha \leq e / d$, write $p_{f, \alpha}=\sum_{i=0}^{d-1} p_{f, \alpha, i} x^{i}$ and $f=\sum_{i=0}^{d} f_{i} x^{i}$. Then

$$
\frac{f(z)-f(x)}{z-x}=\sum_{i=0}^{d} f_{i} \frac{z^{i}-x^{i}}{z-x}=\sum_{i=0}^{d} q_{i}(z) x^{i}
$$


with $q_{i}=\sum_{l=0}^{d-1-i} f_{i+l+1} z^{l}$. By Corollary 2.10, $p_{f, \alpha, i}=\operatorname{Res}\left[\begin{array}{c}p q_{i} \mathrm{~d} z \\ f^{\alpha+1}\end{array}\right]$ for $i=0, \ldots, d-1$. We have that $\operatorname{deg}\left(q_{i}\right) \leq d-i-1$ and $\mathrm{h}_{1}\left(q_{i}\right) \leq \mathrm{h}_{1}(f)$. Hence $\operatorname{deg}\left(p q_{i}\right) \leq e+d-i-1$ and $\mathrm{h}_{1}\left(p q_{i}\right) \leq \mathrm{h}_{1}(p)+\mathrm{h}_{1}(f)$. By Theorem 3.2, $f_{d}^{e+1-\alpha(d-1)} p_{f, \alpha, i} \in \mathbb{Z}$ and

$$
\log \left|f_{d}^{e+1-\alpha(d-1)} p_{f, \alpha, i}\right| \leq \mathrm{h}_{1}(p)+\mathrm{h}_{1}(f)+(e-i-\alpha d) \mathrm{h}(f)+(e-i) \log (2),
$$

as stated.

To extend the bounds in Theorem 3.2 to rational functions, we need the following particular case of the arithmetic Nullstellensatz. Given two polynomials $f_{0}, f_{1} \in \mathbb{Z}[x]$ of respective degrees $d_{0}$ and $d_{1}$, we denote by $S\left(f_{0}, f_{1}\right) \in \mathbb{Q}^{\left(d_{0}+d_{1}\right) \times\left(d_{0}+d_{1}\right)}$ their Sylvester matrix, and by $\sigma\left(f_{0}, f_{1}\right)=\operatorname{det}\left(S\left(f_{0}, f_{1}\right)\right)$ their Sylvester resultant. If $f_{0}$ and $f_{1}$ are coprime, this Sylvester resultant is nonzero.

Lemma 3.9. Let $f_{0}, f_{1} \in \mathbb{Z}[x]$ be coprime polynomials. Then there exist $p_{0}, p_{1} \in \mathbb{Z}[x]$ such that

$$
\sigma\left(f_{0}, f_{1}\right)=p_{0} f_{0}+p_{1} f_{1}
$$

satisfying, for $i=0,1$,

(1) $\operatorname{deg}\left(p_{i}\right)+\operatorname{deg}\left(f_{i}\right) \leq \operatorname{deg}\left(f_{0}\right)+\operatorname{deg}\left(f_{1}\right)-1$,

(2) $\mathrm{h}_{1}\left(p_{i}\right)+\mathrm{h}_{1}\left(f_{i}\right) \leq \operatorname{deg}\left(f_{1}\right) \mathrm{h}_{1}\left(f_{0}\right)+\operatorname{deg}\left(f_{0}\right) \mathrm{h}_{1}\left(f_{1}\right)$.

Moreover, $\log \left|\sigma\left(f_{0}, f_{1}\right)\right| \leq \operatorname{deg}\left(f_{1}\right) \mathrm{h}_{1}\left(f_{0}\right)+\operatorname{deg}\left(f_{0}\right) \mathrm{h}_{1}\left(f_{1}\right)$.

Proof. Set $d_{i}=\operatorname{deg}\left(f_{i}\right)$ for short. Since $f_{0}$ and $f_{1}$ are coprime, there are unique $q_{0}, q_{1} \in \mathbb{Q}[x]$ with $\operatorname{deg}\left(q_{0}\right) \leq d_{1}-1$ and $\operatorname{deg}\left(q_{1}\right) \leq d_{0}-1$ such that

$$
1=q_{0} f_{0}+q_{1} f_{1} .
$$

This Bézout identity translates into a square system of linear equations over $\mathbb{Q}$. Such system can be written down as

$$
S\left(f_{0}, f_{1}\right) \cdot Q=b
$$

with $b \in \mathbb{Q}^{d_{0}+d_{1}}$ a vector with an entry equal to 1 , that corresponding to the constant term in $(3.9)$, and all others ones equal to 0 , and $Q \in \mathbb{Q}^{d_{0}+d_{1}}$ the vector of coefficients of $q_{0}$ and $q_{1}$.

Set $p_{i}=\sigma\left(f_{0}, f_{1}\right) q_{i}, i=0,1$. The upper bound for the degree of $p_{i}$ follows from this construction. By Cramer's rule, the coefficients of $p_{i}$ are $\left(d_{0}+d_{1}-1\right)$-minors of $S\left(f_{0}, f_{1}\right)$. The upper bounds for $\sigma\left(f_{0}, f_{1}\right)$ and for the length of the $p_{i}$ 's can be verified by analyzing these minors.

Theorem 3.10. Let $f \in \mathbb{Z}[x] \backslash \mathbb{Z}$ and $f_{0}, g \in \mathbb{Z}[x]$ with $f_{0}$ coprime with $f_{0}$. Set $d=\operatorname{deg}(f), d_{0}=\operatorname{deg}\left(f_{0}\right)$ and $e=\operatorname{deg}(g)$, and let $f_{d}$ be the leading coefficient of $f$. Then, for $\alpha \in \mathbb{N}$,

$$
\sigma\left(f, f_{0}\right)^{\alpha+1} f_{d}^{e+\alpha+1} \operatorname{Res}\left[\begin{array}{c}
g / f_{0} \mathrm{~d} x \\
f^{\alpha+1}
\end{array}\right] \in \mathbb{Z}
$$

and

$$
\begin{aligned}
\log \left|\sigma\left(f, f_{0}\right)^{\alpha+1} f_{d}^{e+\alpha+1} \operatorname{Res}\left[\begin{array}{c}
g / f_{0} \mathrm{~d} x \\
f^{\alpha+1}
\end{array}\right]\right| & \leq \mathrm{h}_{1}(g)+((\alpha+1) d-1) \mathrm{h}_{1}\left(f_{0}\right) \\
& +\left(e+(\alpha+1) d_{0}\right) \mathrm{h}_{1}(f)+(e+\alpha d) \log (2)
\end{aligned}
$$


Proof. The polynomials $f_{0}$ and $f^{\alpha+1}$ are coprime of degrees $d_{0}$ and $(\alpha+1) d$, and lengths $\mathrm{h}_{1}\left(f_{0}\right)$ and $\mathrm{h}_{1}\left(f^{\alpha+1}\right) \leq(\alpha+1) \mathrm{h}_{1}(f)$.

By the multiplicativity of the Sylvester resultant,

$$
\sigma\left(f^{\alpha+1}, f_{0}\right)=\sigma\left(f, f_{0}\right)^{\alpha+1} .
$$

Set $\gamma=\sigma\left(f, f_{0}\right)$ for short. By Lemma 3.9 , there are $p_{\alpha, 0}, p_{\alpha, 1} \in \mathbb{Z}[x]$ such that

$$
\gamma^{\alpha+1}=p_{\alpha, 0} f_{0}+p_{\alpha, 1} f^{\alpha+1}
$$

with $\operatorname{deg}\left(p_{\alpha, 0}\right) \leq(\alpha+1) d-1$ and $\mathrm{h}_{1}\left(p_{\alpha, 0}\right) \leq((\alpha+1) d-1) \mathrm{h}_{1}\left(f_{0}\right)+(\alpha+1) d_{0} \mathrm{~h}_{1}(f)$. By (3.11), we have the congruence on the open subset $\mathbb{A}_{\mathbb{Q}}^{1} \backslash V\left(f_{0}\right)$

$$
\frac{g}{f_{0}} \equiv \frac{p_{\alpha, 0} g}{\gamma^{\alpha+1}} \quad\left(\bmod f^{\alpha+1}\right) \text {. }
$$

Since this open subset is a neighborhood of $V(f)$, by Proposition 2.5 .

$$
\operatorname{Res}\left[\begin{array}{c}
g / f_{0} \mathrm{~d} x \\
f^{\alpha+1}
\end{array}\right]=\frac{1}{\gamma^{\alpha+1}} \operatorname{Res}\left[\begin{array}{c}
p_{\alpha, 0} g \mathrm{~d} x \\
f^{\alpha+1}
\end{array}\right] .
$$

Using that $\operatorname{deg}\left(p_{\alpha, 0} g\right) \leq(\alpha+1) d-1+e$, it follows from Theorem 3.2 that $\gamma^{\alpha+1} f_{d}^{e+\alpha+1}$ is a denominator for the residue in the left-hand side of (3.12). Similarly, the bound in (3.10) follows also from Theorem 3.2, using that

$$
\mathrm{h}_{1}\left(p_{\alpha, 0} g\right) \leq \mathrm{h}_{1}\left(p_{\alpha, 0}\right)+\mathrm{h}_{1}(g) \leq((\alpha+1) d-1) \mathrm{h}_{1}\left(f_{0}\right)+(\alpha+1) d_{0} \mathrm{~h}_{1}(f)+\mathrm{h}_{1}(g) .
$$

\section{RESIDUES ON AN AFFINE VARIETY: POLYNOMIALS IN SEPARATED VARIABLES}

In this section, we bound the residues on an affine variety with respect to a family of univariate polynomials in separated variables.

We first extend the different notions of size of polynomials to the multivariate case. As in $\S 2$, we denote by $\boldsymbol{x}$ the group of variables $\left(x_{1}, \ldots, x_{n}\right)$. For $f \in \mathbb{C}[\boldsymbol{x}]$, we adopt the usual notation

$$
f=\sum_{\alpha} f_{\alpha} x^{\alpha}
$$

where, for each index $\boldsymbol{\alpha}=\left(\alpha_{1}, \ldots, \alpha_{n}\right) \in \mathbb{N}^{n}, f_{\boldsymbol{\alpha}}$ denotes an element of $\mathbb{C}$ and $\boldsymbol{x}^{\boldsymbol{\alpha}}$ the monomial $x_{1}^{\alpha_{1}} \ldots x_{n}^{\alpha_{n}}$. The support of $f$, denoted by $\operatorname{supp}(f)$, is the finite subset $\boldsymbol{\alpha}^{\prime}$ 's such that $f_{\boldsymbol{\alpha}} \neq 0$. For each $\boldsymbol{\alpha} \in \mathbb{N}^{n}$, we set $|\boldsymbol{\alpha}|=\sum_{i=1}^{n} \alpha_{i}$ for its length and coeff $\boldsymbol{\alpha}(f)=f_{\boldsymbol{\alpha}}$ for the coefficient of $\boldsymbol{x}^{\boldsymbol{\alpha}}$ in the monomial expansion of $f$. For $\boldsymbol{\alpha}, \boldsymbol{\beta} \in \mathbb{N}^{r}$, we denote by $\langle\boldsymbol{\alpha}, \boldsymbol{\beta}\rangle=\sum_{i=1}^{r} \alpha_{i} \beta_{i}$ their scalar product.

Definition 4.1. Let $f=\sum_{\boldsymbol{\alpha}} f_{\boldsymbol{\alpha}} \boldsymbol{x}^{\alpha} \in \mathbb{Z}[\boldsymbol{x}]$. The (logarithmic) height, length and Mahler measure of $f$ are respectively defined as

$$
\mathrm{h}(f)=\log \left(\max _{\boldsymbol{\alpha}}\left|f_{\boldsymbol{\alpha}}\right|\right), \quad \mathrm{h}_{1}(f)=\log \left(\sum_{\boldsymbol{\alpha}}\left|f_{\boldsymbol{\alpha}}\right|\right) \quad \text { and } \quad \mathrm{m}(f)=\int_{\left(S^{1}\right)^{n}} \log |f| \mathrm{d} \mu^{n},
$$

where $S^{1}=\{z \in \mathbb{C}|| z \mid=1\}$ is the unit circle of $\mathbb{C}$, and $\mu$ the probability Haar measure on it.

These quantities are related by the inequalities

$$
\mathrm{h}(f) \leq \mathrm{h}_{1}(f) \leq \mathrm{h}(f)+\log (n+1) \operatorname{deg}(f), \quad|\mathrm{m}(f)-\mathrm{h}(f)| \leq \log (n+1) \operatorname{deg}(f),
$$

see for instance [DKS13, Lemma 2.30]. 
The projective space $\mathbb{P}_{\mathbb{Q}}^{n}$ has a standard structure of toric variety. Using Arakelov geometry, one can define a notion of canonical height for its equidimensional subvarieties, as explained in [BPS14, Chapter 1]. Alternatively, this height can be defining using Chow forms and a limit procedure à la Tate as in [DP99], see also [DKS13, §2.3].

For an equidimensional projective variety $V \subset \mathbb{P}_{\mathbb{Q}}^{n}$, its canonical height is denoted by $\mathrm{h}(V)$. It is a nonnegative real number that measures the arithmetic complexity of $V$, and that can also be considered as an arithmetic analogue of its degree.

When $V$ is of dimension zero, its canonical height coincides with the sum of the Weil heights of its points. In the other extreme, $\mathrm{h}\left(\mathbb{P}_{\mathbb{Q}}^{n}\right)=0$. When $V$ is a hypersurface, its canonical height is the Mahler measure of its primitive defining polynomial, see for instance [DKS13, Proposition 2.39].

Definition 4.2. For an equidimensional affine variety $X \subset \mathbb{A}_{\mathbb{Q}}^{n}$, we define its degree and height, respectively denote by

$$
\operatorname{deg}(X) \text { and } \mathrm{h}(X),
$$

as the degree and the canonical height of the closure of the image of $X$ by the standard inclusion $\iota: \mathbb{A}_{\mathbb{Q}}^{n} \hookrightarrow \mathbb{P}_{\mathbb{Q}}^{n}$ given by $\iota\left(x_{1}, \ldots, x_{n}\right)=\left(1: x_{1}: \cdots: x_{n}\right)$.

Given a field extension $\mathbb{Q} \hookrightarrow K$, we set

$$
X_{K}=X \times \operatorname{Spec}(K)
$$

for the subvariety of $\mathbb{A}_{K}^{n}$ obtained from $X$ by base change. Set also $\mathbf{1}=(1, \ldots, 1) \in \mathbb{N}^{r}$ and, for $i=1, \ldots, r$, let $\epsilon_{i} \in \mathbb{N}^{r}$ denote the $i$-th vector in the standard basis of $\mathbb{R}^{r}$.

The following is the main result of this section.

Theorem 4.3. Let $X \subset \mathbb{A}_{\mathbb{Q}}^{n}$ be a variety of pure dimension $r \geq 1$ such that

$$
\#\left(X_{\mathbb{C}} \cap V\left(x_{1}, \ldots, x_{r}\right)\right)=\operatorname{deg}(X) .
$$

Let $f_{i} \in \mathbb{Z}\left[x_{i}\right] \backslash \mathbb{Z}, i=1, \ldots, r$, and $g \in \mathbb{Z}\left[x_{1}, \ldots, x_{n}\right]$. Set $\boldsymbol{d}=\left(d_{1}, \ldots, d_{r}\right) \in \mathbb{N}^{r}$ with $d_{i}=\operatorname{deg}\left(f_{i}\right)$ and $e=\operatorname{deg}(g)$, and let $f_{i, d_{i}}$ be the leading coefficient of $f_{i}, i=1, \ldots, r$. Then there exists $\gamma \in \mathbb{Z} \backslash\{0\}$ with

$$
\log |\gamma| \leq e(\mathrm{~h}(X)+\operatorname{deg}(X)(n+2) \log (2 n+3))
$$

such that, for $\boldsymbol{\alpha}=\left(\alpha_{1}, \ldots, \alpha_{r}\right) \in \mathbb{N}^{r}$,

$$
\gamma \cdot\left(\prod_{i=1}^{r} f_{i, d_{i}}^{e+r-\left\langle\boldsymbol{\alpha}+\mathbf{1}, \boldsymbol{d}-\epsilon_{i}\right\rangle}\right) \cdot \operatorname{Res}_{X_{\mathbb{C}}}\left[\begin{array}{c}
g \mathrm{~d} x_{1} \wedge \cdots \wedge \mathrm{d} x_{r} \\
f_{1}^{\alpha_{1}+1}, \ldots, f_{r}^{\alpha_{r}+1}
\end{array}\right] \in \mathbb{Z}
$$

and

$$
\begin{gathered}
\log \left|\gamma \cdot\left(\prod_{i=1}^{r} f_{i, d_{i}}^{e+r-\left\langle\boldsymbol{\alpha}+\mathbf{1}, \boldsymbol{d}-\epsilon_{i}\right\rangle}\right) \cdot \operatorname{Res}_{X_{\mathbb{C}}}\left[\begin{array}{c}
g \mathrm{~d} x_{1} \wedge \cdots \wedge \mathrm{d} x_{r} \\
f_{1}^{\alpha_{1}+1}, \ldots, f_{r}^{\alpha_{r}+1}
\end{array}\right]\right| \leq \mathrm{h}_{1}(g) \\
+(e+r-\langle\boldsymbol{\alpha}+\mathbf{1}, \boldsymbol{d}\rangle) \sum_{i=1}^{r} \mathrm{~h}\left(f_{i}\right)+e \mathrm{~h}(X)+e \operatorname{deg}(X)(n+3) \log (2 n+3) . \\
\text { If } e<\langle\boldsymbol{\alpha}+\mathbf{1}, \boldsymbol{d}\rangle-r, \text { then } \operatorname{Res}_{X_{\mathbb{C}}}\left[\begin{array}{c}
g \mathrm{~d} x_{1} \wedge \cdots \wedge \mathrm{d} x_{r} \\
f_{1}^{\alpha_{1}+1}, \ldots, f_{r}^{\alpha_{r}+1}
\end{array}\right]=0 .
\end{gathered}
$$

When $X$ is the affine space, we have the following more precise result. 
Theorem 4.4. Let $f_{i} \in \mathbb{Z}\left[x_{i}\right] \backslash \mathbb{Z}, i=1, \ldots, n, g \in \mathbb{Z}\left[x_{1}, \ldots, x_{n}\right]$ and $\boldsymbol{\alpha}=\left(\alpha_{1}, \ldots, \alpha_{n}\right) \in$ $\mathbb{N}^{n}$. Set $\boldsymbol{d}=\left(d_{1}, \ldots, d_{n}\right) \in \mathbb{N}^{n}$ with $d_{i}=\operatorname{deg}\left(f_{i}\right)$ and $e=\operatorname{deg}(g)$, and let $f_{i, d_{i}}$ be the leading coefficient of $f_{i}, i=1, \ldots, n$. Then

$$
\left(\prod_{i=1}^{n} f_{i, d_{i}}^{e+n-\left\langle\boldsymbol{\alpha}+\mathbf{1}, \boldsymbol{d}-\epsilon_{i}\right\rangle}\right) \cdot \operatorname{Re}_{\mathbb{A}_{\mathbb{C}}^{n}}\left[\begin{array}{c}
g \mathrm{~d} x_{1} \wedge \cdots \wedge \mathrm{d} x_{n} \\
f_{1}^{\alpha_{1}+1}, \ldots, f_{n}^{\alpha_{n}+1}
\end{array}\right] \in \mathbb{Z}
$$

and

$$
\begin{array}{r}
\log \left|\left(\prod_{i=1}^{n} f_{i, d_{i}}^{e+n-\left\langle\boldsymbol{\alpha}+\mathbf{1}, \boldsymbol{d}-\epsilon_{i}\right\rangle}\right) \cdot \operatorname{Res}_{\mathbb{A}_{\mathbb{C}}^{n}}\left[\begin{array}{c}
g \mathrm{~d} x_{1} \wedge \cdots \wedge \mathrm{d} x_{n} \\
f_{1}^{\alpha_{1}+1}, \ldots, f_{n}^{\alpha_{n}+1}
\end{array}\right]\right| \\
\leq \mathrm{h}_{1}(g)+(e+n-\langle\boldsymbol{\alpha}+\mathbf{1}, \boldsymbol{d}\rangle) \sum_{i=1}^{r} \mathrm{~h}\left(f_{i}\right)+(e-|\boldsymbol{d}|+n) \log (2) .
\end{array}
$$

If $e<\langle\boldsymbol{\alpha}+\mathbf{1}, \boldsymbol{d}\rangle-n$, then $\operatorname{Re}_{\mathbb{A}_{\mathbb{C}}^{n}}\left[\begin{array}{c}g \mathrm{~d} x_{1} \wedge \cdots \wedge \mathrm{d} x_{n} \\ f_{1}^{\alpha_{1}+1}, \ldots, f_{n}^{\alpha_{n}+1}\end{array}\right]=0$.

We give the proof of these theorems after some definitions and auxiliary results. We first extend the bound for the coefficients of Laurent expansions (Corollary 3.7) to our current multivariate setting. Let $\boldsymbol{f}=\left(f_{1}, \ldots, f_{r}\right)$ with $f_{i} \in \mathbb{Z}\left[x_{i}\right] \backslash \mathbb{Z}$, and $\boldsymbol{\alpha}=\left(\alpha_{1}, \ldots, \alpha_{r}\right) \in \mathbb{N}^{r}$. Consider the multivariate Laurent series of $\boldsymbol{f}^{-\boldsymbol{\alpha}-\boldsymbol{1}}$ given by the product of univariate Laurent series of the $f_{i}^{-\alpha_{i}-1}$ 's around the point at infinity: with notation as in (3.7),

$$
\begin{aligned}
\boldsymbol{f}^{-\boldsymbol{\alpha}-\mathbf{1}}=\prod_{i=1}^{r} f_{i}^{-\alpha_{i}-1}=\prod_{i=1}^{r} \sum_{l_{i} \in \mathbb{N}} c_{f_{i}, \alpha_{i}, l_{i}} x_{i}^{-\alpha_{i} d_{i}-l_{i}} & \\
& =\sum_{\boldsymbol{l} \in \mathbb{N}^{r}} c_{\boldsymbol{f}, \boldsymbol{\alpha}, \boldsymbol{l}} x_{1}^{-\left(\alpha_{1}+1\right) d_{1}-l_{1}} \ldots x_{r}^{-\left(\alpha_{r}+1\right) d_{r}-l_{r}}
\end{aligned}
$$

with $c_{\boldsymbol{f}, \boldsymbol{\alpha}, \boldsymbol{l}}=\prod_{i=1}^{r} c_{f_{i}, \alpha_{i}, l_{i}}$. This series is convergent for $\left(x_{1}, \ldots, x_{r}\right) \in \mathbb{C}^{r}$ such that $\left|x_{i}\right|>\max _{\xi \in V\left(f_{i}\right)}|\xi|$ for all $i$.

The following bound is a direct consequence of Corollary 3.7 .

Proposition 4.5. Let $\boldsymbol{f}=\left(f_{1}, \ldots, f_{r}\right)$ with $f_{i} \in \mathbb{Z}\left[x_{i}\right] \backslash \mathbb{Z}$ and $\boldsymbol{\alpha}=\left(\alpha_{1}, \ldots, \alpha_{r}\right) \in \mathbb{N}^{r}$. Set $\boldsymbol{d}=\left(d_{1}, \ldots, d_{r}\right) \in \mathbb{N}^{r}$ with $d_{i}=\operatorname{deg}\left(f_{i}\right)$, and let $f_{i, d_{i}}$ be the leading coefficient of $f_{i}$, $i=1, \ldots, r$. Then the coefficients of the Laurent expansion of $\boldsymbol{f}^{-\boldsymbol{\alpha}-\mathbf{1}}$ in 4.2 satisfy

$$
\left(\prod_{i=1}^{r} f_{i, d_{i}}^{l_{i}+\alpha_{i}+1}\right) c_{\boldsymbol{f}, \boldsymbol{\alpha}, l} \in \mathbb{Z}
$$

and

$$
\log \left|\left(\prod_{i=1}^{r} f_{i, d_{i}}^{l_{i}+\alpha_{i}+1}\right) c_{\boldsymbol{f}, \boldsymbol{\alpha}, l}\right| \leq \sum_{i=1}^{r} l_{i} \mathrm{~h}\left(f_{i}\right)+(|\boldsymbol{l}|+\langle\boldsymbol{\alpha}, \boldsymbol{d}\rangle) \log (2) .
$$

For the remainder of this section, we assume that $X \subset \mathbb{A}_{\mathbb{Q}}^{n}$ is a variety of pure dimension $r \geq 1$ and that

$$
\#\left(X_{\mathbb{C}} \cap V\left(x_{1}, \ldots, x_{r}\right)\right)=\operatorname{deg}(X) .
$$

Set also $\boldsymbol{x}^{\prime}=\left(x_{1}, \ldots, x_{r}\right)$.

For $g \in \mathbb{Z}\left[x_{1}, \ldots, x_{n}\right]$, we consider the trace function $\Theta_{X, \boldsymbol{x}^{\prime}, g}$ as in (2.12). By Proposition 2.13, this is a polynomial in the variables $\boldsymbol{y}=\left(y_{1}, \ldots, y_{r}\right)$ with rational coefficients, and these coefficients are given by the residue multi-sequence of the polynomial 
$r$-form $g \mathrm{~d} \boldsymbol{x}^{\prime}:$ for $\boldsymbol{\alpha}=\left(\alpha_{1}, \ldots, \alpha_{r}\right) \in \mathbb{N}^{r}$,

$$
\operatorname{coeff}_{\boldsymbol{\alpha}}\left(\Theta_{X, \boldsymbol{x}^{\prime}, g}\right)=\operatorname{Res}_{X_{\mathbb{C}}}\left[\begin{array}{c}
g \mathrm{~d} x_{1} \wedge \cdots \wedge \mathrm{d} x_{r} \\
x_{1}^{\alpha_{1}+1}, \ldots, x_{r}^{\alpha_{r}+1}
\end{array}\right] .
$$

Moreover, $\operatorname{deg}\left(\Theta_{X, \boldsymbol{x}^{\prime}, g}\right) \leq \operatorname{deg}(g)$. We next bound these coefficients, by applying the arithmetic Perron theorem in [DKS13].

Lemma 4.6. Let $X, \boldsymbol{x}^{\prime}$ and $g$ be as above, and $\Theta_{X, \boldsymbol{x}^{\prime} g} \in \mathbb{Q}[\boldsymbol{y}]$ the associated trace function. Then there exists $\gamma \in \mathbb{Z} \backslash\{0\}$ with $\gamma \Theta_{X, \boldsymbol{x}^{\prime} g} \in \mathbb{Z}\left[x_{1}, \ldots, x_{r}\right]$ such that

$$
\begin{aligned}
\log |\gamma| & \leq \operatorname{deg}(g)(\mathrm{h}(X)+\operatorname{deg}(X)(n+2) \log (2 n+3)), \\
\mathrm{h}\left(\gamma \Theta_{X, \boldsymbol{x}^{\prime} g}\right) & \leq \mathrm{h}_{1}(g)+\operatorname{deg}(g)(\mathrm{h}(X)+\operatorname{deg}(X)(n+2) \log (2 n+3)) .
\end{aligned}
$$

Proof. Set $e=\operatorname{deg}(g)$ and $D=\operatorname{deg}(X)$ for short. If $e=0$, then $g \in \mathbb{Z}$ and $\Theta_{X, \boldsymbol{x}^{\prime}, g}=$ $D g$, and the statement is clear. Hence, we can suppose without loss of generality that $e \geq 1$.

Write $\boldsymbol{x}^{\prime}=\left(x_{1}, \ldots, x_{r}\right)$ and $\boldsymbol{x}^{\prime \prime}=\left(x_{r+1}, \ldots, x_{n}\right)$, so that $\boldsymbol{x}=\left(\boldsymbol{x}^{\prime}, \boldsymbol{x}^{\prime \prime}\right)$. Consider the map

$$
\pi: X \longrightarrow \mathbb{A}_{\mathbb{Q}}^{r}, \quad \boldsymbol{x} \longmapsto \boldsymbol{x}^{\prime} .
$$

Its fiber at 0 coincides with the intersection $X \cap V\left(\boldsymbol{x}^{\prime}\right)$. By hypothesis, this fiber is of cardinality $\operatorname{deg}(X)$ and so this is a finite map, see for instance [KPS01, Lemma 2.14]. Hence, the map

$$
\operatorname{id}_{\mathbb{A}^{r}} \times \pi: \mathbb{A}_{\mathbb{Q}}^{n-r} \times X \longrightarrow \mathbb{A}_{\mathbb{Q}}^{n-r} \times \mathbb{A}_{\mathbb{Q}}^{r}
$$

is also finite of degree $D$. Let $\boldsymbol{u}=\left(u_{r+1}, \ldots, u_{n}\right)$ be a group of $n-r$ variables and set

$$
p=g+\sum_{i=r+1}^{n} u_{i} x_{i} \in \mathbb{Z}[\boldsymbol{u}, \boldsymbol{x}] .
$$

Set $\boldsymbol{y}=\left(y_{1}, \ldots, y_{r}\right)$ and let $E \in \mathbb{Z}[\boldsymbol{u}, \boldsymbol{y}, t]$ be an irreducible polynomial giving a minimal equation of integral dependence for $p$ with respect to this map.

We have that $\operatorname{deg}_{t}(E) \leq D$. On the other hand, $p$ separates the points of the fiber of $\pi$ at 0 , which implies that $\operatorname{deg}_{t}(E) \geq D$. Hence,

$$
\operatorname{deg}_{t}(E)=D
$$

and so this minimal polynomial coincides, up to a scalar factor, with the characteristic polynomial of $p$ with respect to the map $\operatorname{id}_{\mathbb{A}^{r}} \times \pi$.

Precisely, if we write $E=\sum_{j=0}^{D} E_{j} t^{j}$ with $E_{j} \in \mathbb{Z}[\boldsymbol{u}, \boldsymbol{y}], j=0, \ldots, D-1$, and $E_{D} \in \mathbb{Z} \backslash\{0\}$, then $E_{D}^{-1} E$ is the characteristic polynomial of $p$ with respect to the map $\operatorname{id}_{\mathbb{A}^{r}} \times \pi$ and $E_{D}^{-1} E(\mathbf{0}, \boldsymbol{y})$ is the characteristic polynomial of $g$ with respect to the map $\pi$. In particular,

$$
\Theta_{X, \boldsymbol{x}^{\prime} g}=-\frac{E_{D-1}(\mathbf{0}, \boldsymbol{y})}{E_{D}} \in \mathbb{Q}[\boldsymbol{y}] .
$$

Setting $\gamma=E_{D} \in \mathbb{Z} \backslash\{0\}$, we have that $\gamma \Theta_{X, \boldsymbol{x}^{\prime}, g} \in \mathbb{Z}[\boldsymbol{y}]$ and $\mathrm{h}\left(\gamma \Theta_{X, \boldsymbol{x}^{\prime}, g}\right) \leq \mathrm{h}\left(E_{D-1}\right)$. Consider now the map

$$
\mathbb{A}_{\mathbb{Q}}^{n-r} \times X \longrightarrow \mathbb{A}_{\mathbb{Q}}^{n-r} \times \mathbb{A}_{\mathbb{Q}}^{r+1}, \quad(\boldsymbol{u}, \boldsymbol{x})=\left(\boldsymbol{u}, \boldsymbol{x}^{\prime}, \boldsymbol{x}^{\prime \prime}\right) \longmapsto\left(\boldsymbol{u}, \boldsymbol{x}^{\prime}, p(\boldsymbol{u}, \boldsymbol{x})\right) .
$$

Its image coincides with the hypersurface defined by $E$. Then [DKS13, Theorem 3.15] implies that

$$
\begin{aligned}
& \mathrm{h}\left(E_{D}\right)+D \mathrm{~h}(g), \mathrm{h}\left(E_{D-1}\right)+(D-1) \mathrm{h}(g) \\
& \leq D \mathrm{~h}(g)+e\left(\mathrm{~h}\left(\mathbb{A}_{\mathbb{Q}}^{r} \times X\right)+\operatorname{deg}\left(\mathbb{A}_{\mathbb{Q}}^{r} \times X\right)(n+2) \log (2 n+3)\right) .
\end{aligned}
$$


By [DKS13, Lemma 3.16], $\operatorname{deg}\left(\mathbb{A}_{\mathbb{Q}}^{r} \times X\right)=\operatorname{deg}(X)=D$ and $\mathrm{h}\left(\mathbb{A}_{\mathbb{Q}}^{r} \times X\right)=\mathrm{h}(X)$. Combining this with 4.4 , we easily derive stated bounds for $\gamma$ and $\Theta_{X, \boldsymbol{x}^{\prime} g}$.

The following lemma reduces the computation of residues on an affine variety with respect to univariate polynomials in separated variables to the monomial case.

Lemma 4.7. With notation as in Theorem 4.3, let $c_{\boldsymbol{f}, \boldsymbol{\alpha}, \boldsymbol{l}}, \boldsymbol{l} \in \mathbb{N}^{r}$, be the coefficients of the Laurent expansion of $\boldsymbol{f}^{-\boldsymbol{\alpha}-\mathbf{1}}$ as in 4.2 . Then

$$
\operatorname{Res}_{X_{\mathbb{C}}}\left[\begin{array}{c}
g \mathrm{~d} x_{1} \wedge \cdots \wedge \mathrm{d} x_{r} \\
f_{1}^{\alpha_{1}+1}, \ldots, f_{r}^{\alpha_{r}+1}
\end{array}\right]=\sum_{l} c_{\boldsymbol{f}, \boldsymbol{\alpha}, l} \operatorname{Res}_{X_{\mathbb{C}}}\left[\begin{array}{c}
g \mathrm{~d} x_{1} \wedge \cdots \wedge \mathrm{d} x_{r} \\
x_{1}^{\left(\alpha_{1}+1\right) d_{1}+l_{1}}, \ldots, x_{r}^{\left(\alpha_{r}+1\right) d_{r}+l_{r}}
\end{array}\right],
$$

the sum being over $\boldsymbol{l} \in \mathbb{N}^{r}$ such that $|\boldsymbol{l}| \leq e-\langle\boldsymbol{\alpha}+\mathbf{1}, \boldsymbol{d}\rangle+r$.

Proof. Let $R>\max _{i} \max _{\xi \in V\left(f_{i}\right)}|\xi|$. The multivariate Laurent series in 4.2 converges uniformly on the $r$-dimensional analytic cycle

$$
\Gamma_{R}=\left\{\boldsymbol{\xi} \in X(\mathbb{C})|| \xi_{1}|=\cdots=| \xi_{r} \mid=R\right\} .
$$

Set for short $\mathrm{d} \boldsymbol{x}^{\prime}=\mathrm{d} x_{1} \wedge \cdots \wedge \mathrm{d} x_{r}$. Then

$$
\begin{aligned}
\operatorname{Res}_{X_{\mathbb{C}}}\left[\begin{array}{l}
g \mathrm{~d} \boldsymbol{x}^{\prime} \\
\left.\boldsymbol{f}^{\boldsymbol{\alpha}+\mathbf{1}}\right]
\end{array}\right] & =\frac{1}{(2 \pi i)^{r}} \int_{\Gamma_{R}} \frac{g(\boldsymbol{x}) \mathrm{d} \boldsymbol{x}^{\prime}}{f_{1}^{\alpha_{1}+1}\left(x_{1}\right) \ldots f_{r}^{\alpha_{r}+1}\left(x_{r}\right)} \\
& =\sum_{\boldsymbol{l} \in \mathbb{N}^{r}} c_{\boldsymbol{f}, \boldsymbol{\alpha}, \boldsymbol{l}} \frac{1}{(2 \pi i)^{r}} \int_{\Gamma_{R}} \frac{g(\boldsymbol{x}) \mathrm{d} \boldsymbol{x}^{\prime}}{x_{1}^{\left(\alpha_{1}+1\right) d_{1}+l_{1}}, \ldots, x_{r}^{\left(\alpha_{r}+1\right) d_{r}+l_{r}}} \\
& =\sum_{\boldsymbol{l} \in \mathbb{N}^{r}} c_{\boldsymbol{f}, \boldsymbol{\alpha}, \boldsymbol{l}} \operatorname{Res}_{X_{\mathbb{C}}}\left[x_{1}^{\left(\alpha_{1}+1\right) d_{1}+l_{1}}, \ldots \mathrm{d} \boldsymbol{x}^{\prime}, x_{r}^{\left(\alpha_{r}+1\right) d_{r}+l_{r}}\right] .
\end{aligned}
$$

The hypothesis 4.3 implies that $\boldsymbol{x}^{\prime}$ has no zeros on $X$ on the hyperplane at infinity. By (2.8) and Theorem 2.7, this implies that the residues in this last sum vanish whenever $e<\langle\boldsymbol{\alpha}+\mathbf{1}, \boldsymbol{d}\rangle+|\boldsymbol{l}|-r$, concluding the proof.

Proof of Theorems 4.3 and 4.4. We first consider the general case, when $X \subset \mathbb{A}_{\mathbb{Q}}^{n}$ is of pure dimension $r$ and $\#\left(X \cap V\left(x_{1}, \ldots, x_{r}\right)\right)=\operatorname{deg}(X)$. Set

$$
\eta=\prod_{i=1}^{r} f_{i, d_{i}}^{e+r-\left\langle\boldsymbol{\alpha}+\mathbf{1}, \boldsymbol{d}-\epsilon_{i}\right\rangle} \in \mathbb{Z} \backslash\{0\} .
$$

Let $\boldsymbol{l} \in \mathbb{N}^{r}$ with $|\boldsymbol{l}| \leq e+r-\langle\boldsymbol{\alpha}+\mathbf{1}, \boldsymbol{d}\rangle$. We have that $l_{i}+\alpha_{i}+1 \leq e+r-\left\langle\boldsymbol{\alpha}+\mathbf{1}, \boldsymbol{d}-\epsilon_{i}\right\rangle$ for all $i$. Hence, Proposition 4.5 implies that $\eta c_{\boldsymbol{f}, \boldsymbol{\alpha}, \boldsymbol{l}} \in \mathbb{Z}$ and

$$
\log \left|\eta c_{\boldsymbol{f}, \boldsymbol{\alpha}, l}\right| \leq(e-\langle\boldsymbol{\alpha}+\mathbf{1}, \boldsymbol{d}\rangle+r) \sum_{i=1}^{r} \mathrm{~h}\left(f_{i}\right)+(e-|\boldsymbol{d}|+r) \log (2) .
$$

Let $\gamma \in \mathbb{Z} \backslash\{0\}$ with $\log |\gamma| \leq e(h(X)+\operatorname{deg}(X)(n+2) \log (2 n+3))$ as in Lemma 4.6 . Set for short

$$
\rho_{\boldsymbol{f}}(g, \boldsymbol{\alpha})=\operatorname{Res}_{X_{\mathbb{C}}}\left[\begin{array}{c}
g \mathrm{~d} x_{1} \wedge \cdots \wedge \mathrm{d} x_{r} \\
f_{1}^{\alpha_{1}+1}, \ldots, f_{r}^{\alpha_{r}+1}
\end{array}\right] .
$$


From the formula in Lemma 4.7 and the bounds in Lemma 4.6 and 4.6), we deduce that $\gamma \eta \rho_{\boldsymbol{f}}(g, \boldsymbol{\alpha}) \in \mathbb{Z}$ and that

$$
\begin{aligned}
\log \left|\gamma \eta \rho_{\boldsymbol{f}}(g, \boldsymbol{\alpha})\right| \leq & \max _{\boldsymbol{l}} \log \left|\eta c_{\boldsymbol{f}, \boldsymbol{\alpha}, \boldsymbol{l}}\right|+\mathrm{h}_{1}(g)+e(\mathrm{~h}(X)+\operatorname{deg}(X)(n+2) \log (2 n+3)) \\
& +\log \left(\#\left\{\boldsymbol{l} \in \mathbb{N}^{r}|| \boldsymbol{l} \mid \leq e+r-\langle\boldsymbol{\alpha}+\mathbf{1}, \boldsymbol{d}\rangle\right\}\right) \\
\leq & (e+r-\langle\boldsymbol{\alpha}+\mathbf{1}, \boldsymbol{d}\rangle) \sum_{i=1}^{r} \mathrm{~h}\left(f_{i}\right)+(e-|\boldsymbol{d}|+r) \log (2)+\mathrm{h}_{1}(g) \\
& +e(\mathrm{~h}(X)+\operatorname{deg}(X)(n+2) \log (2 n+3)) \\
& +(e+r-\langle\boldsymbol{\alpha}+\mathbf{1}, \boldsymbol{d}\rangle) \log (r+1) \\
\leq & \mathrm{h}_{1}(g)+(e+r-\langle\boldsymbol{\alpha}+\mathbf{1}, \boldsymbol{d}\rangle) \sum_{i=1}^{r} \mathrm{~h}\left(f_{i}\right)+e \mathrm{~h}(X) \\
& +e \operatorname{deg}(X)(n+3) \log (2 n+3) .
\end{aligned}
$$

If $e<\langle\boldsymbol{\alpha}+\mathbf{1}, \boldsymbol{d}\rangle-r$, by Theorem 2.7 all the residues in the sum in the right-hand side of the the formula 4.5 vanish. Hence $\rho_{\boldsymbol{f}}(g, \boldsymbol{\alpha})=0$, proving the last statement.

If $X=\mathbb{A}_{\mathbb{Q}}^{n}$, then $\Theta_{X, \boldsymbol{f}, g}=\operatorname{Tr}_{\mathbb{C}(\boldsymbol{x}) / \mathbb{C}(\boldsymbol{x})}(g)=g(\boldsymbol{y})$. Hence, in this case we can take $\gamma=1$ and we have that $\mathrm{h}_{1}\left(\Theta_{X, \boldsymbol{x}^{\prime} g}\right)=\mathrm{h}_{1}(g)$. Thus

$$
\begin{aligned}
\log \left|\eta \rho_{\boldsymbol{f}}(g, \boldsymbol{\alpha})\right| & \leq \mathrm{h}_{1}(g)+\max _{\boldsymbol{l}} \log \left|\eta c_{\boldsymbol{f}, \boldsymbol{\alpha}, l}\right| \\
& \leq \mathrm{h}_{1}(g)+(e+r-\langle\boldsymbol{\alpha}+\mathbf{1}, \boldsymbol{d}\rangle) \sum_{i=1}^{r} \mathrm{~h}\left(f_{i}\right)+(e-|\boldsymbol{d}|+r) \log (2),
\end{aligned}
$$

as stated.

For completeness, we also extend the bounds for the coefficients of the $f$-adic expansions (Proposition 3.8) to our current multivariate setting. Let $\boldsymbol{f}=\left(f_{1}, \ldots, f_{r}\right)$ with $f_{i} \in \mathbb{Z}\left[x_{i}\right] \backslash \mathbb{Z}$. Given $p \in \mathbb{Z}\left[\boldsymbol{x}^{\prime}\right]=\mathbb{Z}\left[x_{1}, \ldots, x_{r}\right]$, its $\boldsymbol{f}$-adic expansion is its unique finite representation as

$$
p=\sum_{\boldsymbol{\alpha} \in \mathbb{N}^{r}} p_{\boldsymbol{f}, \boldsymbol{\alpha}} \boldsymbol{f}^{\boldsymbol{\alpha}}
$$

with $p_{\boldsymbol{f}, \boldsymbol{\alpha}} \in \mathbb{Q}\left[\boldsymbol{x}^{\prime}\right]$ such that $\operatorname{deg}_{x_{i}}\left(p_{\boldsymbol{f}, \boldsymbol{\alpha}}\right) \leq \operatorname{deg}\left(f_{i}\right)-1$ for all $\boldsymbol{\alpha}$ and $i$. Using the Bergman-Weil formula (Theorem 2.15), these coefficients can be expressed in terms of residues as

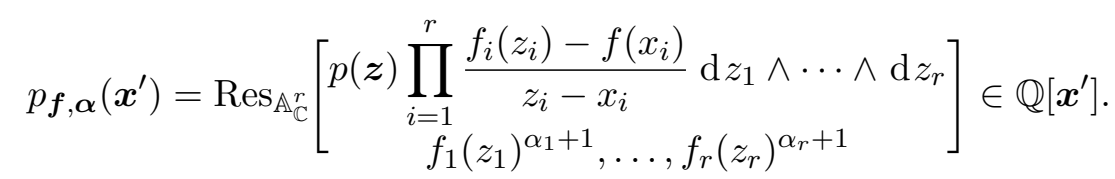

Proposition 4.8. Let $\boldsymbol{f}=\left(f_{1}, \ldots, f_{r}\right)$ with $f_{i} \in \mathbb{Z}\left[x_{i}\right] \backslash \mathbb{Z}$ and $p \in \mathbb{Z}\left[x_{1}, \ldots, x_{r}\right]$. Set $\boldsymbol{d}=\left(d_{1}, \ldots, d_{r}\right)$ with $d_{i}=\operatorname{deg}\left(f_{i}\right)$ and $\boldsymbol{e}=\left(e_{1}, \ldots, e_{r}\right)$ with $e_{i}=\operatorname{deg}_{x_{i}}(p)$, and let $f_{i, d_{i}}$ be the leading coefficient of $f_{i}, i=1, \ldots, r$. Then

$$
\left(\prod_{i=1}^{r} f_{i, d_{i}}^{e_{i}+1-\alpha_{i}\left(d_{i}-1\right)}\right) \cdot p_{\boldsymbol{f}, \boldsymbol{\alpha}} \in \mathbb{Z}\left[x_{1}, \ldots, x_{r}\right]
$$

and

$$
\mathrm{h}_{1}\left(\left(\prod_{i=1}^{r} f_{i, d_{i}}^{e_{i}+1-\alpha_{i}\left(d_{i}-1\right)}\right) \cdot p_{\boldsymbol{f}, \boldsymbol{\alpha}}\right) \leq \mathrm{h}_{1}(p)+\sum_{i=1}^{r}\left(e_{i}-\alpha_{i} d_{i}\right) \mathrm{h}_{1}\left(f_{i}\right)+|\boldsymbol{e}| \log (2) .
$$

If $e_{i}<\alpha_{i} d_{i}$ for some $i$, then $p_{\boldsymbol{f}, \boldsymbol{\alpha}}=0$. 
Proof. Consider first the case when $p$ is a monomial, that is, $p=\left(\boldsymbol{x}^{\prime}\right)^{\boldsymbol{\beta}}$ with $\boldsymbol{\beta}=$ $\left(\beta_{1}, \ldots, \beta_{r}\right) \in \mathbb{N}^{r}$. Since this is a product of polynomials in separated variables, its $\boldsymbol{f}$-adic expansion can be obtained by multiplying the $f_{i}$-adic expansion of its factors. Hence, for $\boldsymbol{\alpha}=\left(\alpha_{1}, \ldots, \alpha_{r}\right) \in \mathbb{N}^{r}$,

$$
p_{\boldsymbol{f}, \boldsymbol{\alpha}}=\prod_{i=1}^{r}\left(x_{i}^{\beta_{i}}\right)_{f_{i}, \alpha_{i}} .
$$

Set $\nu=\prod_{i=1}^{r} f_{i, d_{i}}^{\beta_{i}+1-\alpha_{i}\left(d_{i}-1\right)} \in \mathbb{Z} \backslash\{0\}$. By 4.9 and Proposition 3.8, $\nu p_{\boldsymbol{f}, \boldsymbol{\alpha}}=$ $\prod_{i=1}^{r}\left(f_{i, d_{i}}^{\beta_{i}+1-\alpha_{i}\left(d_{i}-1\right)}\left(x_{i}^{\beta_{i}}\right)_{f_{i}, \alpha_{i}}\right) \in \mathbb{Z}\left[\boldsymbol{x}^{\prime}\right]$ and

$$
\mathrm{h}_{1}\left(\nu p_{\boldsymbol{f}, \boldsymbol{\alpha}}\right) \leq \sum_{i=1}^{r} \mathrm{~h}_{1}\left(f_{i, d_{i}}^{\beta_{i}+1-\alpha_{i}\left(d_{i}-1\right)}\left(x_{i}^{\beta_{i}}\right)_{f_{i}, \alpha_{i}}\right) \leq \sum_{i=1}^{r}\left(\beta_{i}-\alpha_{i} d_{i}\right) \mathrm{h}_{1}\left(f_{i}\right)+\beta_{i} \log (2),
$$

proving (4.7) and (4.8) in this case. Moreover, if $e_{i}<\alpha_{i} d_{i}$ for some $i$, then $\left(x_{i}^{\beta_{i}}\right)_{f_{i}, \alpha_{i}}=0$ and so $p_{\boldsymbol{f}, \boldsymbol{\alpha}}=0$, giving also the last statement in this case.

The case of an arbitrary $p$ follows by linearity from the monomial one.

\section{An ARITHMEtic Elimination theOREM}

Let $f_{1}, \ldots, f_{n} \in \mathbb{Z}\left[x_{1}, \ldots, x_{n}\right]$ be polynomials with a finite number of common zeros in $\overline{\mathbb{Q}}^{n}$. A classical method to solve the system of equations

$$
f_{1}=\cdots=f_{n}=0
$$

is to eliminate variables, that is, to find $\phi_{l} \in \mathbb{Z}\left[x_{l}\right] \backslash\{0\}, l=1, \ldots, n$, and $a_{l, i} \in$ $\mathbb{Z}\left[x_{1}, \ldots, x_{n}\right], l, i=1, \ldots, n$, such that

$$
\phi_{l}=\sum_{i=1}^{n} a_{l, i} f_{i} .
$$

Applying a variant of his approach to the effective Nullstellensatz, Jelonek has obtained an optimal upper bound for the degrees of these polynomials [Jel05, Theorem 1.6]. Here we prove an arithmetic analogue of this result, bounding the height of the $\phi_{l}$ 's and the $a_{l, i}$ 's. Our proof proceeds by adapting Jelonek's approach and applying the tools from arithmetic intersection theory in DKS13. Our main result in this section (Theorem 5.1) is an arithmetic analogue of the "generalized elimination theorem" in JJel05, Theorem 4.3].

Given a variety $X \subset \mathbb{A}_{\mathbb{Q}}^{n}$, a polynomial relation is said to hold on $X$ if it holds modulo the ideal of definition $I(X)$ or, equivalently, if it holds for every point of $X(\overline{\mathbb{Q}})$.

Theorem 5.1. Let $X \subset \mathbb{A}_{\mathbb{Q}}^{n}$ be a variety of pure dimension $r \geq 0, f_{1}, \ldots, f_{s} \in$ $\mathbb{Z}\left[x_{1}, \ldots, x_{n}\right] \backslash \mathbb{Z}$ with $s \leq r$, and $q \in \mathbb{Z}\left[x_{1}, \ldots, x_{n}\right]$ a polynomial that is constant on every irreducible component of $X_{\overline{\mathbb{Q}}} \cap V\left(f_{1}, \ldots, f_{s}\right)$. Set $d_{j}=\operatorname{deg}\left(f_{j}\right), j=1, \ldots, s$. Then there exist $\phi \in \mathbb{Z}[t] \backslash\{0\}$ and $a_{1}, \ldots, a_{s} \in \mathbb{Z}\left[x_{1}, \ldots, x_{n}\right]$ such that

$$
\phi(q)=a_{1} f_{1}+\cdots+a_{s} f_{s} \quad \text { on } X
$$


satisfying, for $i=1, \ldots, s$,

$$
\begin{aligned}
& \operatorname{deg}(\phi) \leq\left(\prod_{j=1}^{s} d_{j}\right) \operatorname{deg}(X) \\
& \operatorname{deg}\left(a_{i}\right)+\operatorname{deg}\left(f_{i}\right) \leq \operatorname{deg}(q)\left(\prod_{j=1}^{s} d_{j}\right) \operatorname{deg}(X) \\
& \mathrm{h}(\phi), \mathrm{h}\left(a_{i}\right)+\mathrm{h}\left(f_{i}\right) \leq \operatorname{deg}(q)\left(\prod_{j=1}^{s} d_{j}\right)\left(\mathrm{h}(X)+\operatorname{deg}(X)\left(\sum_{j=1}^{s} \frac{\mathrm{h}\left(f_{j}\right)}{d_{j}}+\frac{\mathrm{h}(q)}{\operatorname{deg}(q)}\right.\right. \\
&\left.\left.\quad+(r+1) \log \left(2(r+2)(n+1)^{2}\right)\right)\right) .
\end{aligned}
$$

We give the proof of this result after some auxiliary lemmas.

Lemma 5.2. Let $X \subset \mathbb{A}_{\mathbb{Q}}^{n}$ be an equidimensional variety and $q \in \mathbb{Z}\left[x_{1}, \ldots, x_{n}\right]$ a polynomial that is constant on every irreducible component of $X_{\overline{\mathbb{Q}}}$. Then there exists $\phi \in \mathbb{Z}[t] \backslash\{0\}$ such that

$$
\phi(q)=0 \quad \text { on } X
$$

with

$$
\operatorname{deg}(\phi) \leq \operatorname{deg}(X) \quad \text { and } \quad \mathrm{m}(\phi) \leq \operatorname{deg}(q) \mathrm{h}(X)+\operatorname{deg}(X) \mathrm{h}_{1}(q-z),
$$

where $z$ is an additional variable. In particular, $\mathrm{h}(\phi) \leq \operatorname{deg}(q) \mathrm{h}(X)+\operatorname{deg}(X)(\mathrm{h}(q)+$ $\operatorname{deg}(q) \log (n+2))$.

Proof. Let $Z=\left\{\zeta_{1}, \ldots, \zeta_{l}\right\} \subset \overline{\mathbb{Q}}$ be the finite set of values of $q$ on $X(\overline{\mathbb{Q}})$. It is invariant under the action of the absolute Galois group $\operatorname{Gal}(\overline{\mathbb{Q}} / \mathbb{Q})$, and so we can consider a primitive polynomial with integer coefficients defined as

$$
\phi=\gamma \prod_{i=1}^{l}\left(t-\zeta_{i}\right) \in \mathbb{Z}[t] \backslash\{0\}
$$

for a suitable $\gamma \in \mathbb{Q}^{\times}$. By construction, $\phi(q)=0$ on $X$. The cardinality of $Z$ is bounded by the number of irreducible components of $X$ and, a fortiori, by its degree. Hence $\operatorname{deg}(\phi) \leq \operatorname{deg}(X)$, which gives the degree bound.

To bound the Mahler measure of $\phi$, consider the hypersurface $V(q-z) \subset \mathbb{A}_{\mathbb{Q}}^{n} \times \mathbb{A}_{\mathbb{Q}}^{1}$, with $z$ the standard coordinate of $\mathbb{A}_{\mathbb{Q}}^{1}$. The variety

$$
Y=\left(X \times \mathbb{A}_{\mathbb{Q}}^{1}\right) \cap V(q-z)
$$

is of dimension $r:=\operatorname{dim}(X)$ and its projection onto the last coordinate coincides with $Z$. Choose a subset $I \subset\{1, \ldots, n\}$ of cardinality $r$ such that the projection $\varpi: \mathbb{A}_{\mathbb{Q}}^{n} \times \mathbb{A}_{\mathbb{Q}}^{1} \rightarrow \mathbb{A}_{\mathbb{Q}}^{r} \times \mathbb{A}_{\mathbb{Q}}^{1}$ defined by

$$
\varpi\left(x_{1}, \ldots, x_{n}, z\right) \longmapsto\left(\left(x_{i}\right)_{i \in I}, z\right)
$$

verifies $\operatorname{dim}(\overline{\varpi(Y)})=r$. Let $\varrho: \mathbb{A}_{\mathbb{Q}}^{r} \times \mathbb{A}_{\mathbb{Q}}^{1} \rightarrow \mathbb{A}_{\mathbb{Q}}^{1}$ be the projection onto the second factor. Then $\varrho(\overline{\varpi(Y)})=Z$ is zero dimensional. The theorem of dimension of fibers then implies that

$$
\overline{\varpi(Y)}=\mathbb{A}_{\mathbb{Q}}^{r} \times Z,
$$

and so $\overline{\varpi(Y)}$ is a hypersurface with defining polynomial $\phi$.

By [DKS13, Corollary 2.61 and Lemma 3.16],

$$
\mathrm{h}(Y) \leq \operatorname{deg}(q) \mathrm{h}(X)+\operatorname{deg}(X) \mathrm{h}_{1}(q-z) .
$$


By [DKS13, Proposition 2.64], we have $\mathrm{h}(\overline{\varpi(Y)}) \leq \mathrm{h}(Y)$. Since $\overline{\varpi(Y)}=V(\phi)$, we have that $\mathrm{h}(\overline{\varpi(Y)})=\mathrm{m}(\phi)$, which completes the proof of $(5.5)$. The last statement follows from the second inequality in (4.1).

Definition 5.3. Let $\varphi: X \rightarrow Y$ be a generically finite dominant map of affine varieties. We say that $\varphi$ is finite at a point $\boldsymbol{y} \in Y$ if there is an open neighborhood $U$ of $\boldsymbol{y}$ such that the restriction of $\varphi$ to a map $\varphi^{-1}(U) \rightarrow U$ is finite.

The following lemma is a variant of [Jel05, Lemma 4.1].

Lemma 5.4. Let $X \subset \mathbb{A}_{\mathbb{Q}}^{n}$ be a variety of pure dimension $r \geq 0, q \in \mathbb{Z}\left[x_{1}, \ldots, x_{n}\right]$ a polynomial that is not constant on any irreducible component of $X_{\overline{\mathbb{Q}}}$, and $u_{i, j}, i=$ $1, \ldots, r-1, j=r, \ldots, n$, a group of $(r-1)(n-r+1)$ variables. Consider the transcendental field extension $\mathbb{K}=\mathbb{Q}\left(\left(u_{i, j}\right)_{i, j}\right)$ and the map

$$
\pi: X_{\mathbb{K}} \longrightarrow \mathbb{A}_{\mathbb{K}}^{r}, \quad \boldsymbol{x}=\left(x_{1}, \ldots, x_{n}\right) \longmapsto\left(x_{1}+\sum_{j=r}^{n} u_{1, j} x_{j}, \ldots, x_{r-1}+\sum_{j=r}^{n} u_{r, j} x_{j}, q(\boldsymbol{x})\right) .
$$

Then $\pi$ is dominant, and there exists $p \in \mathbb{Z}\left[t_{r}\right] \backslash\{0\}$ such that $\pi$ is finite on $\mathbb{A}_{\mathbb{K}}^{r} \backslash V(p)$.

Proof. Consider the map $\varrho: X \rightarrow \mathbb{A}_{\mathbb{Q}}^{1}$ given by $\boldsymbol{x} \mapsto q(\boldsymbol{x})$ and let $\mathcal{X}$ be its generic fiber. In algebraic terms, this map corresponds to the morphism of $\mathbb{Q}$-algebras $\mathbb{Q}[z] \rightarrow$ $\mathbb{Q}\left[x_{1}, \ldots, x_{n}\right] / I(X)$ defined by $z \mapsto q$, and $\mathcal{X}$ is the subvariety of $\mathbb{A}_{\mathbb{Q}(g)}^{n}$ defined by the ideal

$$
I(\mathcal{X})=\mathbb{Q}(q) I(X) \subset \mathbb{Q}(q)\left[x_{1}, \ldots, x_{n}\right] .
$$

The hypothesis that the polynomial $q$ is not constant on any irreducible component of $X_{\mathbb{Q}}$ implies that the map $\varrho$ is surjective and has no vertical fibers. Hence, $\mathcal{X}$ is of pure dimension $r-1$ and the natural morphism

$$
\mathbb{Q}\left[x_{1}, \ldots, x_{n}\right] / I(X) \longrightarrow \mathbb{Q}(q)\left[x_{1}, \ldots, x_{n}\right] / I(\mathcal{X})
$$

is an inclusion. Let $\mathcal{X}_{\mathbb{K}}$ be the affine variety obtained by base change, which is the subvariety of $\mathbb{A}_{\mathbb{K}(g)}^{n}$ corresponding to the ideal $I\left(\mathcal{X}_{\mathbb{K}}\right)=\mathbb{K}(q) I(X) \subset \mathbb{K}(q)\left[x_{1}, \ldots, x_{n}\right]$.

For $i=1, \ldots, r-1$, set $\ell_{i}=u_{i, r} x_{r}+\cdots+u_{i, n} x_{n} \in \mathbb{K}\left[x_{r}, \ldots, x_{n}\right]$. Then the map

$$
\mathcal{X}_{\mathbb{K}} \longrightarrow \mathbb{A}_{\mathbb{K}(q)}^{r-1}, \quad\left(x_{1}, \ldots, x_{r-1}, \boldsymbol{x}^{\prime}\right) \longmapsto\left(x_{1}+\ell_{1}\left(\boldsymbol{x}^{\prime}\right), \ldots, x_{r-1}+\ell_{r-1}\left(\boldsymbol{x}^{\prime}\right)\right)
$$

is dominant and finite, since it is the restriction to $\mathcal{X}_{\mathbb{K}}$ of a general linear map $\mathbb{A}_{\mathbb{K}(q)}^{n} \rightarrow$ $\mathbb{A}_{\mathbb{K}(g)}^{r-1}$ in reduced triangular form.

Choose $\ell_{r} \in \mathbb{Q}\left[x_{r}, \ldots, x_{n}\right]$ a sufficiently generic linear form, so that the map

$$
X_{\mathbb{K}} \longrightarrow \mathbb{A}_{\mathbb{K}}^{r}, \quad\left(x_{1}, \ldots, x_{r-1}, \boldsymbol{x}^{\prime}\right) \longmapsto\left(x_{1}+\ell_{1}\left(\boldsymbol{x}^{\prime}\right), \ldots, x_{r-1}+\ell_{r-1}, \ell_{r}\left(\boldsymbol{x}^{\prime}\right)\right)
$$

is also finite, and let $P \in \mathbb{Q}(q)\left[t_{1}, \ldots, t_{r-1}\right][T]$ be a polynomial giving an equation of integral dependence of $\ell_{r}$ with respect to the map in (5.6). Let $p \in \mathbb{Q}\left[t_{r}\right] \backslash\{0\}$ so that $p(q)$ is a denominator of $P$. Then $\ell_{r}$ integral with respect to the map $\pi$ on the open subset $\mathbb{A}_{\mathbb{K}}^{r} \backslash V(p)$.

Since the map in (5.7) is finite, this implies that $\pi$ is finite outside $V(p)$. Since $\operatorname{dim}\left(X_{\mathbb{K}}\right)=r=\operatorname{dim}\left(\mathbb{A}_{\mathbb{K}}^{r}\right)$, this map is also dominant, completing the proof.

Proof of Theorem 5.1. First we treat the case when $q$ is not constant on any of the irreducible components of $X_{\overline{\mathbb{Q}}}$. Let $z$ be an additional variable, consider the map

$$
\varphi: X \times \mathbb{A}_{\mathbb{Q}}^{1} \longrightarrow \mathbb{A}_{\mathbb{Q}}^{n} \times \mathbb{A}_{\mathbb{Q}}^{s}, \quad(\boldsymbol{x}, z) \mapsto\left(\boldsymbol{x}, z f_{1}(\boldsymbol{x}), \ldots, z f_{s}(\boldsymbol{x})\right)
$$


and let $W \subset \mathbb{A}_{\mathbb{Q}}^{n} \times \mathbb{A}_{\mathbb{Q}}^{s}$ be the closure of its image. The assumptions on the polynomial $q$ imply that no irreducible component of $X$ is contained in the zero set of the $f_{j}$ 's, and so $W$ is of pure dimension $r+1$. By construction, $\varphi$ gives an isomorphism between $X$ and $W$ outside the zero set of the $f_{j}$ 's.

Let $\left\{\zeta_{1}, \ldots, \zeta_{l}\right\} \subset \overline{\mathbb{Q}}$ be the finite $\operatorname{Gal}(\overline{\mathbb{Q}} / \mathbb{Q})$-invariant subset of values of the polynomial $q$ on the irreducible components of $X_{\overline{\mathbb{Q}}} \cap V\left(f_{1}, \ldots, f_{s}\right)$ and set

$$
\theta_{1}=\gamma \prod_{i=1}^{l}\left(t-\zeta_{i}\right) \in \mathbb{Z}[t]
$$

for a suitable $\gamma \in \mathbb{Q}^{\times}$such that $\theta_{1}$ is primitive. The hypersurface $H:=V\left(\theta_{1} \circ q\right) \subset \mathbb{A}_{\mathbb{Q}}^{n}$ contains the variety $X \cap V\left(f_{1}, \ldots, f_{s}\right)$ and so the restricted map

$$
\varphi:(X \backslash H) \times \mathbb{A}_{\mathbb{Q}}^{1} \longrightarrow W \backslash\left(H \times \mathbb{A}_{\mathbb{Q}}^{s}\right)
$$

is an isomorphism. Since $q$ is not constant on any of the irreducible components of $X_{\overline{\mathbb{Q}}}$, the hypersurface $H$ contains no irreducible component of $X$.

For $i=1, \ldots, r$, let $\boldsymbol{u}_{i}=\left(u_{i, 1}, \ldots, u_{i, n}\right)$ be a group of $n$ variables and consider the general linear form

$$
\ell_{i}=u_{i, 1} x_{1}+\cdots+u_{i, n} x_{n} \in \mathbb{Q}\left(\boldsymbol{u}_{i}\right)\left[x_{1}, \ldots, x_{n}\right] .
$$

Set $\boldsymbol{u}=\left(\boldsymbol{u}_{1}, \ldots, \boldsymbol{u}_{r}\right)$, and consider the field $\mathbb{K}=\mathbb{Q}(\boldsymbol{u})$ and the map

$$
\pi: W_{\mathbb{K}} \longrightarrow \mathbb{A}_{\mathbb{K}}^{r+1}, \quad(\boldsymbol{x}, \boldsymbol{z}) \longmapsto\left(z_{1}+\ell_{1}(\boldsymbol{x}), \ldots, z_{s}+\ell_{r}(\boldsymbol{x}), \ell_{s+1}(\boldsymbol{x}), \ldots, \ell_{r}(\boldsymbol{x}), q(\boldsymbol{x})\right) .
$$

By Lemma 5.4 this map is dominant, and there exists $\theta_{2} \in \mathbb{Z}\left[y_{r+1}\right] \backslash\{0\}$ such that it is finite on the open subset $\mathbb{A}_{\mathbb{K}}^{r+1} \backslash V\left(\theta_{2}\right)$.

Let $\varphi_{\mathbb{K}}$ be the base change of the map in $(5.8)$ given by the field extension $\mathbb{Q} \hookrightarrow \mathbb{K}$ and set $\theta=\theta_{1}\left(y_{r+1}\right) \theta_{2}\left(y_{r+1}\right) \in \mathbb{Z}\left[y_{r+1}\right]$. Then the composition

$$
\psi=\pi \circ \varphi_{\mathbb{K}}: X_{\mathbb{K}} \times \mathbb{A}_{\mathbb{K}}^{1} \longrightarrow \mathbb{A}_{\mathbb{K}}^{r+1}
$$

is finite on the open subset $\mathbb{A}_{\mathbb{K}}^{r+1} \backslash V(\theta)$.

Set $\boldsymbol{y}=\left(y_{1}, \ldots, y_{r+1}\right)$. For convenience, we also set $f_{j}=0, j=s+1, \ldots, r$. Then the previous condition is equivalent to the fact that the inclusion of $\mathbb{Q}(\boldsymbol{u})$-algebras

$$
\psi^{\#}: \mathbb{K}[\boldsymbol{y}]_{\theta} \longrightarrow\left(\mathbb{K}[x] / I\left(X_{\mathbb{K}}\right)\right)_{\theta \circ q} \otimes \mathbb{K}[z], \quad y_{i} \longmapsto \begin{cases}z f_{i}+\ell_{i} & \text { if } 1 \leq i \leq r, \\ q & \text { if } i=r+1,\end{cases}
$$

is integral. Let $E \in \mathbb{K}[\boldsymbol{y}]_{\theta}[z]$ be the minimal polynomial of the variable $z$ with respect to $\psi^{\#}$. It is unique up to a unit of the ring $\mathbb{K}[\boldsymbol{y}]_{\theta}$. We choose it as a primitive and squarefree polynomial in $\mathbb{Z}[\boldsymbol{u}][\boldsymbol{y}, z]$ and write it is as

$$
E=\sum_{j=0}^{\delta} \sum_{\boldsymbol{\alpha} \in \mathbb{N}^{r+1}} E_{\boldsymbol{\alpha}, j} \boldsymbol{y}^{\boldsymbol{\alpha}} z^{\delta-j}
$$

with $\delta=\operatorname{deg}_{z}(E)$ and $E_{\boldsymbol{\alpha}, j} \in \mathbb{Z}[\boldsymbol{u}]$ for all $\boldsymbol{\alpha}, j$. The coefficient of $z^{\delta}$ is

$$
\widetilde{\phi}=\sum_{\boldsymbol{\alpha} \in \mathbb{N}^{r+1}} E_{\boldsymbol{\alpha}, 0} \boldsymbol{y}^{\boldsymbol{\alpha}} .
$$

Since the morphism $\psi^{\#}$ in 5.10$)$ is integral, $\widetilde{\phi}=\lambda \theta^{k}\left(y_{r+1}\right)$ with $\lambda \in \mathbb{Z}[\boldsymbol{u}]$ and $k \in \mathbb{N}$. 
By definition,

$$
\begin{aligned}
E\left(z, z f_{1}\right. & \left.+\ell_{1}, \ldots, z f_{r}+\ell_{r}, q\right) \\
& =\sum_{j=0}^{\delta} \sum_{\boldsymbol{\alpha} \in \mathbb{N}^{r+1}} E_{\boldsymbol{\alpha}, j}\left(\prod_{k=1}^{r}\left(z f_{k}+\ell_{k}\right)^{\alpha_{i}}\right) q^{\alpha_{r+1}} z^{\delta-j}=0 \quad \text { on } X_{\mathbb{K}} \times \mathbb{A}_{\mathbb{K}}^{1} .
\end{aligned}
$$

Hence, all the coefficients in the expansion of this expression with respect to $z$ vanish identically on $X_{\mathbb{K}}$. We will extract the relation $(5.1)$ from the coefficient of $z^{\delta}$. Set, for $i=1, \ldots, s$,

$$
\widetilde{a}_{i}=-\sum_{j=1}^{\delta} \sum_{\boldsymbol{\alpha} \in \mathbb{N}^{r+1}} \sum_{\boldsymbol{\beta} \in B_{i, j, \boldsymbol{\alpha}}} E_{\boldsymbol{\alpha}, j}\left(\prod_{k=1}^{r}\left(\begin{array}{c}
\alpha_{k} \\
\beta_{k}
\end{array}\right) \ell_{k}^{\alpha_{k}-\beta_{k}}\right) f_{1}^{\beta_{1}} \ldots f_{i-1}^{\beta_{i-1}} f_{i}^{\beta_{i}-1} q^{\alpha_{r+1}} \in \mathbb{Z}[\boldsymbol{u}, x],
$$

where the indexing set $B_{i, j, \boldsymbol{\alpha}}$ consists of the vectors $\boldsymbol{\beta}=\left(\beta_{1}, \ldots, \beta_{r}\right)$ with $|\boldsymbol{\beta}|=j$ and $\beta_{k} \leq \alpha_{k}$ for $1 \leq k \leq i, \beta_{i} \geq 1$, and $\beta_{k}=0$ for $i+1 \leq k \leq r$. It follows from (5.11) that

$$
\widetilde{\phi}(q)=\widetilde{a}_{1} f_{1}+\cdots+\widetilde{a}_{s} f_{s} \quad \text { on } X_{\mathbb{K}} .
$$

The relation (5.1) is obtained by taking the coefficient of some nonzero term in the monomial expansion with respect to $\boldsymbol{u}$ of the left-hand side of this relation.

To control the degree and height of the minimal polynomial $E$, we relate it to the implicit equation of the image of a polynomial map. Indeed, by [DKS13, Lemma 4.8] applied to the map $\psi$ in $(5.9)$, the polynomial $E$ gives also an equation for the closure of the image of the map

$$
X_{\mathbb{K}(z)} \longrightarrow \mathbb{A}_{\mathbb{K}(z)}^{r+1}, \quad(\boldsymbol{x}, z) \longmapsto\left(z f_{1}(\boldsymbol{x})+\ell_{1}(\boldsymbol{x}), \ldots, z f_{r}(\boldsymbol{x})+\ell_{r}(\boldsymbol{x}), q(\boldsymbol{x})\right) .
$$

Note that [DKS13, Lemma 4.8] is stated finite maps, but it also holds for generically finite maps, with the same proof, and so we can apply it to the map $\psi$ to prove the claim above.

Hence, we can bound the size of $E$ by applying the arithmetic Perron theorem. To this end, we summarize the partial degrees, height and number of monomials of the polynomials defining the map in (5.12). Set $d_{j}=\operatorname{deg}\left(f_{j}\right)$ and $h_{j}=\mathrm{h}\left(f_{j}\right), j=1, \ldots, s$ and, for convenience, we set also $d_{j}=1$ and $h_{j}=0, j=s+1, \ldots, r$. For $j=1, \ldots, r$, we have

(1) $\operatorname{deg}_{\boldsymbol{x}}\left(z f_{j}+\ell_{j}\right) \leq d_{j}$

(2) $\operatorname{deg}_{\boldsymbol{u}, z}\left(z f_{j}+\ell_{j}\right)=1$,

(3) $\mathrm{h}\left(z f_{j}+\ell_{j}\right) \leq h_{j}$

(4) $\log \left(\# \operatorname{supp}\left(z f_{j}+\ell_{j}\right)+2\right) \leq d_{j} \log (2 n+3)$.

Set $e=\operatorname{deg}_{\boldsymbol{x}}(q)$ and $l=\mathrm{h}(q)$. We have $\operatorname{deg}_{\boldsymbol{u}, z}(q)=0$, and the number of parameters, that is the auxiliary variables $\boldsymbol{u}, z$, is $r n+1$.

Write

$$
E=\sum_{\boldsymbol{\alpha} \in \mathbb{N}^{r+1}} E_{\boldsymbol{\alpha}} \boldsymbol{y}^{\boldsymbol{\alpha}}
$$

with $E_{\boldsymbol{\alpha}} \in \mathbb{Z}[\boldsymbol{u}, z] \backslash\{0\}$, and consider the vectors in $\mathbb{N}^{r+1}$ given by

$$
\boldsymbol{d}=\left(d_{1}, \ldots, d_{s}, 1, \ldots, 1, e\right), \quad \boldsymbol{\delta}=(1, \ldots, 1,0), \quad \boldsymbol{h}=\left(h_{1}, \ldots, h_{s}, 0, \ldots, 0, l\right) .
$$

By [DKS13, Theorem 3.15], for all $\boldsymbol{\alpha} \in \operatorname{supp}(E)$,

$$
\langle\boldsymbol{d}, \boldsymbol{\alpha}\rangle \leq e\left(\prod_{j=1}^{s} d_{j}\right) \operatorname{deg}(X), \quad \operatorname{deg}_{\boldsymbol{u}, z}\left(E_{\boldsymbol{\alpha}}\right)+\langle\boldsymbol{\delta}, \boldsymbol{\alpha}\rangle \leq r e\left(\prod_{j=1}^{s} d_{j}\right) \operatorname{deg}(X)
$$


and

$$
\begin{aligned}
\mathrm{h}\left(E_{\boldsymbol{\alpha}}\right)+\langle\boldsymbol{h}, \boldsymbol{\alpha}\rangle \leq & e\left(\prod_{j=1}^{s} d_{j}\right)\left(\mathrm{h}(X)+\operatorname{deg}(X)\left(\sum_{j=1}^{r} \frac{\left.h_{j}+d_{j} \log (2 n+3)\right)+\log (r n+2)}{d_{j}}\right.\right. \\
& \left.\left.+\frac{l+\log (\# \operatorname{supp}(q)+2)}{e}+\log (r+2)\right)\right) \\
\leq & e\left(\prod_{j=1}^{s} d_{j}\right)\left(\mathrm{h}(X)+\operatorname{deg}(X)\left(\sum_{j=1}^{s} \frac{h_{j}}{d_{j}}+r \log ((2 n+3)(r n+2))\right.\right. \\
& \left.\left.+\frac{l}{e}+\log ((n+3)(r+2))\right)\right) .
\end{aligned}
$$

From (5.13) we get $e \operatorname{deg}_{y_{r+1}}(\widetilde{\phi}) \leq e\left(\prod_{j=1}^{s} d_{j}\right) \operatorname{deg}(X)$, which proves (5.2). Again by (5.13), for $i=1, \ldots, s$,

$$
\operatorname{deg}_{\boldsymbol{x}}\left(\widetilde{a}_{i}\right)+\operatorname{deg}\left(f_{i}\right) \leq \max _{\boldsymbol{\alpha} \in \operatorname{supp}(E)}\langle\boldsymbol{d}, \boldsymbol{\alpha}\rangle \leq e\left(\prod_{j=1}^{s} d_{j}\right) \operatorname{deg}(X)
$$

which proves (5.3). The bound for the height of $\widetilde{\phi}$ follows from (5.14). For the height of the $\widetilde{a}_{i}$ 's we have, for $i=1, \ldots, s$,

$$
\begin{aligned}
\sum_{j=1}^{\delta} \sum_{\substack{\boldsymbol{\alpha} \in \mathbb{N}^{r+1} \\
\boldsymbol{\alpha}_{, j} \neq 0}} \sum_{\boldsymbol{\beta} \in B_{i, j, \boldsymbol{\alpha}}} \prod_{k=1}^{r}\left(\begin{array}{c}
\alpha_{k} \\
\beta_{k}
\end{array}\right) \leq \sum_{\boldsymbol{\alpha} \in \operatorname{supp}(E)} \sum_{\beta \leq \boldsymbol{\alpha}} \prod_{k=1}^{r}\left(\begin{array}{c}
\alpha_{k} \\
\beta_{k}
\end{array}\right) \\
=\sum_{\boldsymbol{\alpha} \in \operatorname{supp}(E)} 2^{\alpha_{1}+\cdots+\alpha_{r}} \leq \# \operatorname{supp}(E) 2^{\operatorname{deg}_{\boldsymbol{y}}(E) .}
\end{aligned}
$$

Hence, by [DKS13, Lemma 2.37(1)],

$$
\begin{aligned}
\mathrm{h}\left(\widetilde{a}_{i}\right)+\mathrm{h}\left(f_{i}\right) \leq \max _{j, \boldsymbol{\alpha}, \boldsymbol{\beta}}\left\{\mathrm{h}\left(E_{\boldsymbol{\alpha}, j}\left(\prod_{k=1}^{r} \ell_{k}^{\alpha_{k}-\beta_{k}}\right) f_{1}^{\beta_{1}} \ldots f_{i-1}^{\beta_{i-1}} f_{i}^{\beta_{i}-1} q^{\alpha_{r+1}}\right)\right\} \\
+\mathrm{h}\left(f_{i}\right)+\log \left(\# \operatorname{supp}(E) 2^{\operatorname{deg}_{\boldsymbol{y}}(E)}\right) .
\end{aligned}
$$

Let $\epsilon_{i} \in \mathbb{N}^{r}$ be the $i$-th vector in the standard basis of $\mathbb{R}^{r}$. By [DKS13, Lemma 2.37(2)], for each $j, \boldsymbol{\alpha}, \beta$ in the maximum in the right-hand side of (5.15), we have

$$
\begin{aligned}
\mathrm{h}\left(E_{\boldsymbol{\alpha}, j}\left(\prod_{k=1}^{r} \ell_{k}^{\alpha_{k}-\beta_{k}}\right) f_{1}^{\beta_{1}} \ldots f_{i-1}^{\beta_{i-1}} f_{i}^{\beta_{i}-1} q^{a_{r+1}}\right) & \leq \mathrm{h}\left(E_{\boldsymbol{\alpha}, j}\right)+|\boldsymbol{\alpha}| \log (n) \\
+ & \left\langle\boldsymbol{h}-\epsilon_{i}, \boldsymbol{\alpha}\right\rangle+\langle\boldsymbol{d}, \boldsymbol{\alpha}\rangle \log (n+1),
\end{aligned}
$$

because $\mathrm{h}_{1}\left(\ell_{k}\right)=\log (n)$ and $\mathrm{h}_{1}\left(f_{j}\right) \leq \mathrm{h}\left(f_{j}\right)+d_{j} \log (n+1)$. It follows from (5.13), (5.14), 5.15) and 5.16) that, for $i=1, \ldots, s$, the sum $\mathrm{h}\left(\widetilde{a}_{i}\right)+\mathrm{h}\left(f_{i}\right)$ is bounded by

$$
e\left(\prod_{j=1}^{s} d_{j}\right)\left(\mathrm{h}(X)+\operatorname{deg}(X)\left(\sum_{j=1}^{s} \frac{h_{j}}{d_{j}}+\frac{l}{e}+(r+1) \log \left(2(r+2)(n+1)^{2}\right)\right)\right),
$$

which gives (5.4). This concludes the case when $q$ is not constant on any of the irreducible components of $X_{\overline{\mathbb{Q}}}$.

For the general case, consider a splitting $X=X_{1} \cup X_{2}$, where $X_{1}$ and $X_{2}$ denote the union of the irreducible components of $X_{\overline{\mathbb{Q}}}$ where $q$ is constant and not constant, 
respectively. Let

$$
\phi_{1}(q)=0 \text { on } X_{1} \quad \text { and } \quad \phi_{2}(q)=a_{1} f_{1}+\cdots+a_{s} f_{s} \text { on } X_{2}
$$

be the equations obtained by applying Lemma 5.2 on $X_{1}$ and the previously considered case on $X_{2}$, respectively. Then

$$
\left(\phi_{1} \phi_{2}\right)(q)=\phi_{1}(q) a_{1} f_{1}+\cdots+\phi_{2}(q) a_{s} f_{s} \text { on } X
$$

is the corresponding equation on $X$. The bounds for the polynomials therein follow readily for those for the polynomials in (5.17).

Remark 5.5. The case when $s>r$, that is, when the number of $f_{i}$ 's exceeds the dimension of the variety $X$, can be reduced to the case when $s \leq r$ considered in Theorem 5.1, by taking linear combinations of the $f_{i}$ 's. The resulting bounds are not so neat since, in particular, the influence of the different $f_{i}$ 's mixes and we loose track of their individual contributions. This case when $s>r$ is not necessary for our present applications, and we omit the formulation of the corresponding bounds.

The next statement is the specialization of Theorem 5.1 to the 0 -dimensional case and a variable $q=x_{l}$.

Corollary 5.6. Let $X \subset \mathbb{A}_{\mathbb{Q}}^{n}$ be a variety of pure dimension $r \geq 0, \boldsymbol{f}=\left(f_{1}, \ldots, f_{r}\right)$ with $f_{i} \in \mathbb{Z}\left[x_{1}, \ldots, x_{n}\right] \backslash \mathbb{Z}$ a family of polynomials defining a complete intersection on $X$. Set $d_{j}=\operatorname{deg}\left(f_{j}\right), j=1, \ldots, r$. Then, for $l=1, \ldots, n$, there exist $\phi_{l} \in \mathbb{Z}\left[x_{l}\right] \backslash\{0\}$ and $a_{l, 1}, \ldots, a_{l, r} \in \mathbb{Z}\left[x_{1}, \ldots, x_{n}\right]$ such that

$$
\phi_{l}=a_{l, 1} f_{1}+\cdots+a_{l, r} f_{r} \quad \text { on } X
$$

satisfying, for $i=1, \ldots, r$,

$$
\begin{aligned}
& \operatorname{deg}\left(\phi_{l}\right), \operatorname{deg}\left(a_{l, i}\right)+\operatorname{deg}\left(f_{i}\right) \leq\left(\prod_{j=1}^{r} d_{j}\right) \operatorname{deg}(X), \\
& \mathrm{h}\left(\phi_{l}\right), \mathrm{h}\left(a_{l, i}\right)+\mathrm{h}\left(f_{i}\right) \leq\left(\prod_{j=1}^{r} d_{j}\right)\left(\mathrm{h}(X)+\operatorname{deg}(X)\left(\sum_{j=1}^{r} \frac{\mathrm{h}\left(f_{j}\right)}{d_{j}}\right.\right. \\
&\left.\left.+(r+1) \log \left(2(r+2)(n+1)^{2}\right)\right)\right) .
\end{aligned}
$$

In the particular case when $X=\mathbb{A}_{\mathbb{Q}}^{n}$, we have $\operatorname{deg}\left(\mathbb{A}_{\mathbb{Q}}^{n}\right)=1$ and $\mathrm{h}\left(\mathbb{A}_{\mathbb{Q}}^{n}\right)=0$. Hence, the previous statement specializes to

$$
\begin{aligned}
& \operatorname{deg}\left(\phi_{l}\right), \operatorname{deg}\left(a_{l, i}\right)+\operatorname{deg}\left(f_{i}\right) \leq \prod_{j=1}^{n} d_{j}, \\
& \mathrm{~h}\left(\phi_{l}\right), \mathrm{h}\left(a_{l, i}\right)+\mathrm{h}\left(f_{i}\right) \leq\left(\prod_{j=1}^{n} d_{j}\right)\left(\sum_{j=1}^{n} \frac{\mathrm{h}\left(f_{j}\right)}{d_{j}}+(n+1) \log \left(2(n+2)(n+1)^{2}\right)\right) .
\end{aligned}
$$

In particular, if $\operatorname{deg}\left(f_{j}\right) \leq d$ and $\mathrm{h}\left(f_{j}\right) \leq h$, then

$$
\begin{aligned}
& \operatorname{deg}\left(\phi_{l}\right), \operatorname{deg}\left(a_{l, i}\right)+\operatorname{deg}\left(f_{i}\right) \leq d^{n}, \\
& \mathrm{~h}\left(\phi_{l}\right), \mathrm{h}\left(a_{l, i}\right)+\mathrm{h}\left(f_{i}\right) \leq n d^{n-1} h+(n+2) \log \left(2(n+2)(n+1)^{2}\right) d^{n} .
\end{aligned}
$$

To prove our bound for residues over an affine variety, we also need the next lemma, allowing to put the variables in general position with a linear change of controlled height. 
Proposition 5.7. Let $X \subset \mathbb{A}_{\mathbb{Q}}^{n}$ be a variety of pure dimension $r$. Then there are affine polynomials $\ell_{i} \in \mathbb{Z}\left[x_{1}, \ldots, x_{n}\right]$ with $\mathrm{h}\left(\ell_{i}\right) \leq 2 \log (\operatorname{deg}(X))+n \log (2)$ such that, for every subset $I \subset\{1, \ldots, n\}$ of cardinality $r$,

$$
\#\left(X_{\overline{\mathbb{Q}}} \cap \bigcap_{i \in I} V\left(\ell_{i}\right)\right)=\operatorname{deg}(X)
$$

In particular, the map $X \rightarrow \mathbb{A}_{\mathbb{Q}}^{r}$ defined by $\left(x_{1}, \ldots, x_{n}\right) \mapsto\left(\ell_{i}\right)_{i \in I}$ is finite of $\operatorname{deg}(X)$.

Proof. For $i=1, \ldots, n$, let $\boldsymbol{u}=\left(u_{i, 1}, \ldots, u_{n, j}\right)$ be a group of $n$ variables. Set $D=$ $\operatorname{deg}(X)$ for short. By [KPS01, Proposition 4.5], there is a polynomial $G \in \mathbb{Q}\left[\boldsymbol{u}_{1}, \ldots, \boldsymbol{u}_{r}\right]$ with $\operatorname{deg}_{\boldsymbol{u}_{i}}(G) \leq 2 D^{2}, i=1, \ldots, r$, such that the condition $G\left(\boldsymbol{b}_{1}, \ldots, \boldsymbol{b}_{r}\right) \neq 0$ for $\boldsymbol{b}_{i} \in \mathbb{Q}^{n+1}, i=1, \ldots, r$, implies that

$$
\#\left(X_{\overline{\mathbb{Q}}} \cap \bigcap_{i=1}^{r} V\left(b_{i, 0}+b_{i, 1} x_{1}+\cdots+b_{i, n} x_{n}\right)\right)=D .
$$

We then set

$$
F:=\prod_{1 \leq i_{1}<\cdots<i_{r} \leq n} G\left(\boldsymbol{u}_{i_{1}}, \ldots, \boldsymbol{u}_{i_{r}}\right) \in \mathbb{Q}\left[\boldsymbol{u}_{1}, \ldots, \boldsymbol{u}_{n}\right] .
$$

This polynomial verifies, for $i=1, \ldots, n$,

$$
\operatorname{deg}_{\boldsymbol{u}_{i}}(F)=\left(\begin{array}{l}
n-1 \\
r-1
\end{array}\right) 2 D^{2} \leq 2^{n} D^{2} .
$$

Hence there are $\boldsymbol{b}_{i} \in \mathbb{Z}^{n+1}, i=1, \ldots, n$, such that $\left|b_{i, j}\right| \leq 2^{n} D^{2}$ for all $i, j$, such that

$$
F\left(\boldsymbol{b}_{1}, \ldots, \boldsymbol{b}_{n}\right) \neq 0 \text {. }
$$

Then the affine polynomials $\ell_{i}=b_{i, 0}+b_{i, 1} x_{1}+\cdots+b_{i, n} x_{n}$ verify that $\mathrm{h}\left(\ell_{i}\right) \leq$ $\log \max _{j}\left|b_{i, j}\right| \leq 2 \log (D)+n \log (2)$ for all $i$ and satisfy the condition (5.18) for every subset subset $I \subset\{1, \ldots, n\}$ of cardinality $r$, proving the first statement.

The second statement follows from the first one, applying [KPS01, Lemma 2.14].

\section{Residues on AN AFFine VARIETy: the General CASE}

In this section, we bound the residue multi-sequence on an affine variety $X \subset \mathbb{A}_{\mathbb{Q}}^{n}$ of dimension $r$, associated to a system of polynomials $\boldsymbol{f}=\left(f_{1}, \ldots, f_{r}\right) \in\left(\mathbb{Z}\left[x_{1}, \ldots, x_{n}\right] \backslash\right.$ $\mathbb{Z})^{r}$ defining a complete intersection on $X$ and a rational $r$-form $\omega$ (Definition 2.4). We also apply these results to bound the coefficients of the representation of a polynomial via the Bergman-Weil trace formula 2.16.

Definition 6.1. Let $\omega$ be a polynomial $r$-form defined over $\mathbb{Z}$ (Definition 2.4). Write $\omega=\sum_{I} g_{I} \mathrm{~d} \boldsymbol{x}_{I}$ with $g_{I} \in \mathbb{Z}\left[x_{1}, \ldots, x_{n}\right]$ for each subset $I \subset\{1, \ldots, n\}$ of cardinality $r$. For each multi-index $I$, write $g_{I}=\sum_{\boldsymbol{\beta}} g_{I, \boldsymbol{\beta}} \boldsymbol{x}^{\boldsymbol{\beta}}$ with $g_{I, \boldsymbol{\beta}} \in \mathbb{Z}, \boldsymbol{\beta} \in \mathbb{N}^{n}$. The degree and the (logarithmic) length of $\omega$ are respectively defined by

$$
\operatorname{deg}(\omega)=\max _{I} \operatorname{deg}\left(g_{I}\right) \quad \text { and } \quad \mathrm{h}_{1}(\omega)=\log \left(\sum_{I, \boldsymbol{\beta}}\left|g_{I, \boldsymbol{\beta}}\right|\right) .
$$

With the previous notation set, for $\boldsymbol{\alpha} \in \mathbb{N}^{r}$,

$$
\rho_{X, \boldsymbol{f}}(\omega, \boldsymbol{\alpha})=\operatorname{Res}_{X_{\mathbb{C}}}\left[\begin{array}{c}
\omega \\
\boldsymbol{f}^{\boldsymbol{\alpha}+\mathbf{1}}
\end{array}\right] .
$$

We first consider these residues for the case when $X$ is good position with respect to a group of variables and $\omega$ is a polynomial multiple of the volume form associated to this group of variables. 
Theorem 6.2. Let $X \subset \mathbb{A}_{\mathbb{Q}}^{n}$ be a variety of pure dimension $r \geq 1$ such that

$$
\#\left(X \cap V\left(x_{1}, \ldots, x_{r}\right)\right)=\operatorname{deg}(X) .
$$

Let $\boldsymbol{f}=\left(f_{1}, \ldots, f_{r}\right)$ be a family of polynomials in $\mathbb{Z}\left[x_{1}, \ldots, x_{n}\right] \backslash \mathbb{Z}$ defining a complete intersection on $X$, and $\omega=g \mathrm{~d} x_{1} \wedge \cdots \wedge \mathrm{d} x_{r}$ with $g \in \mathbb{Z}\left[x_{1}, \ldots, x_{n}\right]$. Set $d_{i}=\operatorname{deg}\left(f_{i}\right)$, $i=1, \ldots, r, e=\operatorname{deg}(g)$ and

$$
D_{X, \boldsymbol{f}}=\operatorname{deg}(X) \prod_{j=1}^{r} d_{j} \quad \text { and } \quad \kappa_{X, \boldsymbol{f}}^{0}=\frac{\mathrm{h}(X)}{\operatorname{deg}(X)}+\sum_{j=1}^{r} \frac{\mathrm{h}\left(f_{j}\right)}{d_{j}}+3(n+2) \log (n+2) .
$$

Then, for $\boldsymbol{\alpha} \in \mathbb{N}^{r}$, there exists $\zeta \in \mathbb{Z} \backslash\{0\}$ such that $\zeta \cdot \rho_{X, \boldsymbol{f}}(\omega, \boldsymbol{\alpha}) \in \mathbb{Z}$ with

$\log |\zeta|, \log \left|\zeta \cdot \rho_{X, \boldsymbol{f}}(\omega, \boldsymbol{\alpha})\right| \leq \mathrm{h}_{1}(g)+(r+1)\left(e+(r+1)(|\boldsymbol{\alpha}|+1) D_{X, \boldsymbol{f}}\right) D_{X, \boldsymbol{f}} \kappa_{X, \boldsymbol{f}}^{0}$.

When $X$ is the affine space, we have the following more precise result.

Theorem 6.3. Let $\boldsymbol{f}=\left(f_{1}, \ldots, f_{n}\right)$ be a family of polynomials in $\mathbb{Z}\left[x_{1}, \ldots, x_{n}\right] \backslash \mathbb{Z}$ defining a complete intersection on $\mathbb{A}_{\mathbb{Q}}^{n}$, and $\omega=g \mathrm{~d} x_{1} \wedge \cdots \wedge \mathrm{d} x_{n}$ a polynomial $n$ form defined over $\mathbb{Z}$. Set $d_{i}=\operatorname{deg}\left(f_{i}\right), i=1, \ldots, n$, and $e=\operatorname{deg}(g)$. For $l=1, \ldots, n$, denote by $\pi_{l}$ the projection $\mathbb{A}_{\mathbb{Q}}^{n} \rightarrow \mathbb{A}_{\mathbb{Q}}^{1}$ to the l-th coordinate of $\mathbb{A}_{\mathbb{Q}}^{n}$ and set

$D_{\boldsymbol{f}}=\prod_{j=1}^{n} d_{j}, \quad \kappa_{\boldsymbol{f}}^{0}=\sum_{j=1}^{n} \frac{\mathrm{h}\left(f_{j}\right)}{d_{j}}+3(n+2) \log (n+2), \quad \Delta_{\boldsymbol{f}}=n D_{\boldsymbol{f}}-\sum_{l=1}^{n} \operatorname{deg}\left(\pi_{l}(V(\boldsymbol{f}))\right)$.

Then there exists $\vartheta \in \mathbb{Z} \backslash\{0\}$ with $\log |\vartheta| \leq n \kappa_{f}^{0}$ such that, for all $\boldsymbol{\alpha} \in \mathbb{N}^{n}$, we have that $\vartheta^{e+(|\boldsymbol{\alpha}|+1)\left(\Delta_{f}+1\right)} \rho_{\mathbb{A}_{\mathbb{Q}}^{n}, \boldsymbol{f}}(\omega, \boldsymbol{\alpha}) \in \mathbb{Z}$ and

$$
\log \left|\vartheta^{e+(|\boldsymbol{\alpha}|+1)\left(\Delta_{\boldsymbol{f}}+1\right)} \rho_{\mathbb{A}_{\mathbb{Q}}^{n}, \boldsymbol{f}}(\omega, \boldsymbol{\alpha})\right| \leq \mathrm{h}_{1}(g)+\left(e+(|\boldsymbol{\alpha}|+1) \Delta_{\boldsymbol{f}}\right) n D_{\boldsymbol{f}} \kappa_{\boldsymbol{f}}^{0} .
$$

Proof of Theorems 6.2 and 6.3. We first consider Theorem 6.2 for an arbitrary affine variety $X \subset \mathbb{A}_{\mathbb{Q}}^{n}$ of pure dimension $r$ satisfying the condition (6.1). For short, we set $\boldsymbol{d}=\left(d_{1}, \ldots, d_{r}\right) \in \mathbb{N}^{r}, D=D_{X, \boldsymbol{f}}$ and $\kappa=\kappa_{X, \boldsymbol{f}}^{0}$.

By Corollary 5.6, there are polynomials $\phi_{l} \in \mathbb{Z}\left[x_{l}\right] \backslash\{0\}$ and $a_{l, i} \in \mathbb{Z}[\boldsymbol{x}]$, for $i, l=$ $1, \ldots, r$, such that $\phi_{l}=\sum_{i=1}^{r} a_{l, i} f_{i}$ for all $l$, with $\operatorname{deg}\left(\phi_{l}\right), \operatorname{deg}\left(a_{l, i}\right)+d_{i} \leq D \operatorname{deg}(X)$ and

$$
\mathrm{h}\left(\phi_{l}\right), \mathrm{h}\left(a_{l, i}\right)+\mathrm{h}\left(f_{i}\right) \leq D\left(\frac{\mathrm{h}(X)}{\operatorname{deg}(X)}+\sum_{j=1}^{r} \frac{\mathrm{h}\left(f_{j}\right)}{d_{j}}+(r+1) \log \left(2(r+2)(n+1)^{2}\right)\right) .
$$

For each $l$, let $\pi_{l}$ be the projection $\mathbb{A}_{\mathbb{Q}}^{n} \rightarrow \mathbb{A}_{\mathbb{Q}}^{1}$ to the $l$-th coordinate of $\mathbb{A}_{\mathbb{Q}}^{n}$. Since $\phi_{l}$ vanishes on $\pi_{l}(X \cap V(\boldsymbol{f}))$ and $\boldsymbol{f}$ is a complete intersection on $X$,

$$
\operatorname{deg}\left(\phi_{l}\right) \geq \operatorname{deg}\left(\pi_{l}(X \cap V(\boldsymbol{f}))\right) \geq 1 .
$$

We next apply the transformation law (Theorem 2.8) to reduce the study of the residue $\rho_{X, \boldsymbol{f}}(\omega, \boldsymbol{\alpha})$ to the case of separated variables, considered in $\$ 4$ Let $\boldsymbol{u}=$ $\left(u_{1}, \ldots, u_{r}\right)$ be a group of $r$ variables. Set $A=\left(a_{l, i}\right)_{l, i} \in \mathbb{Z}[\boldsymbol{x}]^{r \times r}, a_{l}=\sum_{i} a_{l, i} u_{i}$ and

$$
H_{l}=\sum_{k=0}^{|\boldsymbol{\alpha}|} \phi_{l}^{k} a_{l}^{|\boldsymbol{\alpha}|-k} \in \mathbb{Z}[\boldsymbol{u}, \boldsymbol{x}] .
$$

Set $H=\operatorname{det}(A) \cdot \prod_{l=1}^{r} H_{l} \in \mathbb{Z}[\boldsymbol{u}, \boldsymbol{x}]$ as in 2.9$)$ and $G=\operatorname{coeff}_{\boldsymbol{u}^{\alpha}}(H(\boldsymbol{u}, \boldsymbol{x})) \in \mathbb{Z}[\boldsymbol{x}]$. By Theorem 2.8

$$
\rho_{X, \boldsymbol{f}}(\omega, \boldsymbol{\alpha})=\operatorname{Res}_{X_{\mathbb{C}}}\left[\begin{array}{c}
g G \mathrm{~d} x_{1} \wedge \cdots \wedge \mathrm{d} x_{r} \\
\phi_{1}^{|\boldsymbol{\alpha}|+1}, \ldots, \phi_{r}^{|\boldsymbol{\alpha}|+1}
\end{array}\right] .
$$


By the expression in (6.5) and the bounds in (6.3), $\operatorname{deg}_{\boldsymbol{x}}\left(H_{l}\right) \leq|\boldsymbol{\alpha}| D$ for all $l$. We also have that $\operatorname{deg}(\operatorname{det}(A)) \leq r D-|\boldsymbol{d}|$. Thus

$$
\operatorname{deg}(g G) \leq e-|\boldsymbol{d}|+(|\boldsymbol{\alpha}|+1) r D .
$$

For the length, using the inequalities in (4.1), the bounds for the height in (6.3) and comparing it with the constant $\kappa$ in 6.2 , we obtain

$$
\mathrm{h}\left(H_{l}\right) \leq \max _{k}\left(k \mathrm{~h}_{1}\left(\phi_{l}\right)+(|\boldsymbol{\alpha}|-k) \mathrm{h}_{1}\left(a_{l}\right)\right)+\log (|\boldsymbol{\alpha}|+1) \leq|\boldsymbol{\alpha}| D \kappa
$$

and $\mathrm{h}_{1}(\operatorname{det}(A)) \leq r \max _{l, i} \mathrm{~h}_{1}\left(a_{l, i}\right)+\log (r !) \leq r D \kappa$. We deduce that

$$
\mathrm{h}_{1}(g G) \leq \mathrm{h}_{1}(g)+(|\boldsymbol{\alpha}|+1) r D \kappa .
$$

We next apply Theorem 4.3 to the residue in the right-hand side of 2.10 , that is, to the $\phi_{i}$ 's instead of the $f_{i}$ 's, the polynomial $g G$ instead of $g$, and the vector $(|\boldsymbol{\alpha}|, \ldots,|\boldsymbol{\alpha}|) \in \mathbb{N}^{r}$. Set $\delta_{l}=\operatorname{deg}\left(\phi_{l}\right)$ and $\boldsymbol{\delta}=\left(\delta_{1}, \ldots, \delta_{r}\right) \in \mathbb{N}^{r}$, and let $\phi_{l, \delta_{l}}$ be the leading coefficient of $\phi_{l}$. With notation as in this result, set

$$
\zeta=\gamma \cdot \prod_{l=1}^{r} \phi_{l, \delta_{l}}^{\operatorname{deg}(g G)+r-(|\boldsymbol{\alpha}|+1)(|\boldsymbol{\delta}|-1)} \in \mathbb{Z} \backslash\{0\} .
$$

Hence $\zeta \cdot \rho_{X, \boldsymbol{f}}(\omega, \boldsymbol{\alpha}) \in \mathbb{Z}$. Moreover, setting

$$
\begin{aligned}
\kappa_{1}=(\operatorname{deg}(g G)+r-(|\boldsymbol{\alpha}|+1)|\boldsymbol{\delta}|) & \sum_{l=1}^{r} \mathrm{~h}\left(\phi_{l}\right) \\
+ & \operatorname{deg}(g G)(\mathrm{h}(X)+\operatorname{deg}(X)(n+3) \log (2 n+3)),
\end{aligned}
$$

we have that $\log |\zeta| \leq \kappa_{1}$ and $\log \left|\zeta \cdot \rho_{X, \boldsymbol{f}}(\omega, \boldsymbol{\alpha})\right| \leq \mathrm{h}_{1}(g G)+\kappa_{1}$. We also have that $d_{i} \geq 1$ for all $i$ and, by (6.4), $\delta_{l} \geq 1$ for all $l$. Hence $|\boldsymbol{d}|,|\boldsymbol{\delta}| \geq r$. Using this together with (6.7), we obtain

$$
\begin{aligned}
\mathrm{h}_{1}(g G)+\kappa_{1} \leq & \mathrm{h}_{1}(g)+(|\boldsymbol{\alpha}|+1) r D \kappa \\
& +(e+(|\boldsymbol{\alpha}|+1) r D)(r D \kappa+\mathrm{h}(X)+\operatorname{deg}(X)(n+3) \log (2 n+3)) \\
\leq & \mathrm{h}_{1}(g)+e(r+1) D \kappa+(|\boldsymbol{\alpha}|+1)(r+1)^{2} D^{2} \kappa,
\end{aligned}
$$

and similarly $\kappa_{1} \leq e(r+1) D \kappa+(|\boldsymbol{\alpha}|+1)(r+1)^{2} D^{2} \kappa$, which gives the bound in the theorem.

In the case $X=\mathbb{A}_{\mathbb{Q}}^{n}$, set $\vartheta=\prod_{i=1}^{n} \phi_{i, \delta_{i}} \in \mathbb{Z} \backslash\{0\}$. By 6.3 , this quantity satisfies the inequality $\log |\vartheta| \leq n D \kappa$ as stated. Set

$$
\lambda=\vartheta^{\operatorname{deg}(g G)+n-(|\boldsymbol{\alpha}|+1)(|\boldsymbol{\delta}|-1)} \rho_{X, \boldsymbol{f}}(\omega, \boldsymbol{\alpha}) .
$$

By Theorem 4.4, $\lambda \in \mathbb{Z}$ and this integer can be bounded by

$$
\begin{aligned}
\log |\lambda| \leq \mathrm{h}_{1}(g G)+(\operatorname{deg}(g G)+n-(|\boldsymbol{\alpha}|+1)|\boldsymbol{\delta}|) & \sum_{l=1}^{n} \mathrm{~h}\left(\phi_{l}\right) \\
& +(\operatorname{deg}(g G)-|\boldsymbol{\delta}|+n) \log (2) .
\end{aligned}
$$

By the inequalities 6.6 and 6.4,

$$
\operatorname{deg}(g G)+n-(|\boldsymbol{\alpha}|+1)(|\boldsymbol{\delta}|-1) \leq e+(|\boldsymbol{\alpha}|+1)(\Delta+1) .
$$


Set also $\mu=\vartheta^{e+(|\boldsymbol{\alpha}|+1)(\Delta+1)} \rho_{X, \boldsymbol{f}}(\omega, \boldsymbol{\alpha})=\lambda \vartheta^{c}$, where the exponent $c \geq 0$ is the difference between both sides of the inequality in (6.8). Hence $\mu \in \mathbb{Z}$ and

$$
\begin{aligned}
\log |\mu| & \leq \mathrm{h}_{1}(g)+(e+(|\boldsymbol{\alpha}|+1) \Delta) \sum_{l=1}^{n} \mathrm{~h}\left(\phi_{l}\right)+(e-|\boldsymbol{d}|+(|\boldsymbol{\alpha}|+1) n D) \log (2) \\
& \leq \mathrm{h}_{1}(g)+(e+(|\boldsymbol{\alpha}|+1) \Delta) n D \kappa,
\end{aligned}
$$

which concludes the proof of Theorem 6.3 .

We apply these results to bound the coefficients in the Bergman-Weil trace formula. Let $\boldsymbol{f}=\left(f_{1}, \ldots, f_{n}\right) \in \mathbb{C}[\boldsymbol{x}]^{n}$ be a complete intersection on $\mathbb{A}_{\mathbb{Q}}^{n}$. Let $\boldsymbol{z}=\left(z_{1}, \ldots, z_{n}\right)$ be a group of variables and, for $i, j=1, \ldots, n$, set

$$
h_{i, j}=\left(f_{j}\left(x_{1}, \ldots, x_{j}, z_{j+1}, \ldots, z_{n}\right)-f_{j}\left(x_{1}, \ldots, x_{j-1}, z_{j}, \ldots, z_{n}\right)\right) /\left(z_{j}-x_{j}\right) .
$$

These are polynomials in $\mathbb{C}[\boldsymbol{x}, \boldsymbol{z}]$ that verify the identity $(2.15)$, namely

$$
f_{i}(\boldsymbol{z})-f_{i}(\boldsymbol{x})=\sum_{j=1}^{n} h_{i, j}(\boldsymbol{x}, \boldsymbol{z})\left(z_{j}-x_{j}\right)
$$

Put $h_{i}=\sum_{j=1}^{n} h_{i, j} \mathrm{~d} y_{j}$ and, for $p \in \mathbb{C}[\boldsymbol{x}]$ and $\boldsymbol{\alpha} \in \mathbb{N}^{n}$, set

$$
p_{\boldsymbol{\alpha}}=\rho_{\mathbb{A}_{\mathbb{C}}^{n}, \boldsymbol{f}}\left(g \bigwedge_{i=1}^{n} h_{i}, \boldsymbol{\alpha}\right) \in \mathbb{C}[\boldsymbol{x}]
$$

for the coefficient corresponding to $\boldsymbol{\alpha}$ in the representation of $p$ given by the BergmanWeil trace formula. These are polynomials of degree bounded by $\sum_{j=1}^{n} \operatorname{deg}\left(f_{j}\right)-n$.

Corollary 6.4. Let $\boldsymbol{f}=\left(f_{1}, \ldots, f_{n}\right)$ be a family of polynomials in $\mathbb{Z}\left[x_{1}, \ldots, x_{n}\right] \backslash \mathbb{Z}$ defining a complete intersection on $\mathbb{A}_{\mathbb{Q}}^{n}$, and $p \in \mathbb{Z}\left[x_{1}, \ldots, x_{n}\right]$. Set $\boldsymbol{d}=\left(d_{1}, \ldots, d_{n}\right) \in$ $\mathbb{N}^{n}$ with $d_{i}=\operatorname{deg}\left(f_{i}\right)$, and $e=\operatorname{deg}(p)$. Set also

$$
D_{\boldsymbol{f}}=\prod_{j=1}^{n} d_{j} \quad \text { and } \quad \kappa_{\boldsymbol{f}}^{\prime \prime}=\sum_{j=1}^{n} \frac{\mathrm{h}\left(f_{j}\right)}{d_{j}}+3(n+2) \log (n+2) .
$$

Then there exists $\vartheta \in \mathbb{Z} \backslash\{0\}$ with $\log |\vartheta| \leq n \kappa$ such that, for $\boldsymbol{\alpha} \in \mathbb{N}^{n}$, the coefficient $p_{\boldsymbol{\alpha}}$ in 6.10 satisfies that $\vartheta^{e+|\boldsymbol{d}|+(|\boldsymbol{\alpha}|+1)\left(n D_{\boldsymbol{f}}+1\right)} p_{\boldsymbol{\alpha}} \in \mathbb{Z}\left[x_{1}, \ldots, x_{n}\right]$ and

$$
\mathrm{h}\left(\vartheta^{e+|\boldsymbol{d}|+(|\boldsymbol{\alpha}|+1)\left(n D_{\boldsymbol{f}}+1\right)} g_{\boldsymbol{\alpha}}\right) \leq \mathrm{h}_{1}(g)+\left(e+|\boldsymbol{d}|+(|\boldsymbol{\alpha}|+1)\left(n D_{\boldsymbol{f}}+1\right)\right) n D_{\boldsymbol{f}} \kappa_{\boldsymbol{f}}^{\prime \prime} .
$$

Proof. With notation as in (6.9), consider the polynomial $n$-form $\omega=p \bigwedge_{j=1}^{n} h_{j}$ in the variables $\boldsymbol{z}$ with coefficients in $\mathbb{Z}[\boldsymbol{x}]$. From $(6.9)$, we verify that $\operatorname{deg}\left(h_{i, j}\right) \leq d_{j}-1$ and $\mathrm{h}_{1}\left(h_{i, j}\right) \leq \mathrm{h}\left(f_{j}\right)$. This implies that

$$
\operatorname{deg}(\omega) \leq|\boldsymbol{d}|-n \quad \text { and } \quad \mathrm{h}_{1}(\omega) \leq \mathrm{h}_{1}(p)+\sum_{k=1}^{n} \mathrm{~h}_{1}\left(f_{j}\right)+|\boldsymbol{d}| \log (2 n+1),
$$

and the result follows then from Theorem 6.3.

To extend our bounds for residues to rational forms, we need the following version of the arithmetic Nullstellensatz. It is a direct application of [DKS13, Theorem 0.1].

Lemma 6.5. Let $X \subset \mathbb{A}_{\mathbb{Q}}^{n}$ be a variety of pure dimension $r$ and $f_{i} \in \mathbb{Z}\left[x_{1}, \ldots, x_{n}\right]$, $i=0, \ldots, r$, polynomials without common zeros in $X$. Set $d_{i}=\operatorname{deg}\left(f_{i}\right), i=0, \ldots, r$. Then there exist $a \in \mathbb{Z} \backslash\{0\}$ and $p \in \mathbb{Z}\left[x_{1}, \ldots, x_{n}\right]$ such that

$$
p f_{0} \equiv a \bmod \left(f_{1}, \ldots, f_{r}\right) \quad \text { on } X
$$


with $\operatorname{deg}(p)+d_{0} \leq\left(\prod_{i=0}^{r} d_{i}\right) \operatorname{deg}(X)$ and

$\log |a|, \mathrm{h}(p)+\mathrm{h}\left(f_{0}\right) \leq\left(\prod_{j=0}^{r} d_{j}\right) \mathrm{h}(X)+\operatorname{deg}(X)\left(\sum_{i=0}^{r}\left(\prod_{j \neq i} d_{j}\right) \mathrm{h}\left(f_{i}\right)+(4 r+8) \log (n+3)\right)$.

The following result bounds the numerator and denominators in a residue multisequence, in the most general case considered in this paper.

Theorem 6.6. Let $X \subset \mathbb{A}_{\mathbb{Q}}^{n}$ be a variety of pure dimension $r \geq 1$ and $\boldsymbol{f}=\left(f_{1}, \ldots, f_{r}\right)$ a family of polynomials in $\mathbb{Z}\left[x_{1}, \ldots, x_{n}\right] \backslash \mathbb{Z}$ defining a complete intersection on $X$. Let $\omega$ be a rational $r$-form defined over $\mathbb{Q}$ that is regular on $X \cap V(\boldsymbol{f})$. Write $\omega=\tau / f_{0}$ with $\tau$ a polynomial $r$-form defined over $\mathbb{Z}$ and $f_{0} \in \mathbb{Z}\left[x_{1}, \ldots, x_{n}\right]$ not vanishing on $X \cap V(\boldsymbol{f})$. Set $d_{i}=\operatorname{deg}\left(f_{i}\right), i=0, \ldots, r$, and $e=\operatorname{deg}(\tau)$. Set also

$D_{X, \boldsymbol{f}}=\operatorname{deg}(X) \prod_{j=1}^{r} d_{j} \quad$ and $\quad \kappa_{X, \boldsymbol{f}}=\frac{\mathrm{h}(X)}{\operatorname{deg}(X)}+\sum_{j=1}^{r} \frac{\mathrm{h}\left(f_{j}\right)}{d_{j}}+4(n+5)^{2} \log ((n+1) \operatorname{deg}(X))$.

Then, for $\boldsymbol{\alpha} \in \mathbb{N}^{r}$, there is $\zeta \in \mathbb{Z} \backslash\{0\}$ such that $\zeta \cdot \rho_{X, \boldsymbol{f}}(\omega, \boldsymbol{\alpha}) \in \mathbb{Z}$ with

$$
\begin{aligned}
\log |\zeta|+ & \mathrm{h}_{1}(\tau), \log \left|\zeta \cdot \rho_{X, \boldsymbol{f}}(\omega, \boldsymbol{\alpha})\right| \leq\left(\begin{array}{l}
n \\
r
\end{array}\right)\left(\mathrm{h}_{1}(\tau)+e(r+1) D_{X, \boldsymbol{f}} \kappa_{X, \boldsymbol{f}}\right. \\
& \left.+(\boldsymbol{\alpha}+1)\left(2(r+1) D_{X, \boldsymbol{f}} \mathrm{h}\left(f_{0}\right)+\left(3 d_{0}+r+1\right) D_{X, \boldsymbol{f}}^{2} \kappa_{X, \boldsymbol{f}}\right)\right) .
\end{aligned}
$$

When $X$ is the affine space, we have the following more precise result.

Theorem 6.7. Let $\boldsymbol{f}=\left(f_{1}, \ldots, f_{n}\right)$ be a family of polynomials in $\mathbb{Z}\left[x_{1}, \ldots, x_{n}\right] \backslash \mathbb{Z}$ defining a complete intersection on $\mathbb{A}_{\mathbb{Q}}^{n}$, and $\omega$ a rational $n$-form that is regular on $V(\boldsymbol{f})$. Write $\omega=\left(g / f_{0}\right) \mathrm{d} x_{1} \wedge \cdots \wedge \mathrm{d} x_{n}$ with $g, f_{0} \in \mathbb{Z}\left[x_{1}, \ldots, x_{n}\right]$ and $f_{0}$ not vanishing on $V(\boldsymbol{f})$. Set $d_{i}=\operatorname{deg}\left(f_{i}\right), i=0, \ldots, n$, and $e=\operatorname{deg}(g)$. Set also

$$
D_{\boldsymbol{f}}=\prod_{j=1}^{n} d_{j} \quad \text { and } \quad \kappa_{\boldsymbol{f}}=\sum_{i=1}^{n} \frac{\mathrm{h}\left(f_{i}\right)}{d_{i}}+(4 n+8) \log (n+3) .
$$

Then, for $\boldsymbol{\alpha} \in \mathbb{N}^{n}$, there exists $\zeta \in \mathbb{Z} \backslash\{0\}$ such that $\zeta \cdot \rho_{\mathbb{A}_{\mathbb{Q}}^{n}, \boldsymbol{f}}(\omega, \boldsymbol{\alpha}) \in \mathbb{Z}$ with

$$
\begin{aligned}
\log |\zeta|, \log \left|\zeta \cdot \rho_{\mathbb{A}_{\mathbb{Q}}^{n}, \boldsymbol{f}}(\omega, \boldsymbol{\alpha})\right| \leq & \mathrm{h}_{1}(g)+e n D_{\boldsymbol{f}} \kappa_{\boldsymbol{f}} \\
& +(|\boldsymbol{\alpha}|+1)\left(D_{\boldsymbol{f}} \mathrm{h}\left(f_{0}\right)+\left(d_{0}+n\right)\left(n D_{\boldsymbol{f}}+1\right) D_{\boldsymbol{f}} \kappa_{\boldsymbol{f}}\right) .
\end{aligned}
$$

Proof of Theorems 6.6 and 6.7. Set for short $D=D_{X, f}, \kappa=\kappa_{X, f}$ and

$$
\theta_{X}=\frac{\mathrm{h}(X)}{\operatorname{deg}(X)}+\sum_{i=1}^{r} \frac{\mathrm{h}\left(f_{i}\right)}{d_{i}}+(4 r+8) \log (n+3) .
$$

By Lemma 6.5, there exist $a \in \mathbb{Z} \backslash\{0\}$ and $p \in \mathbb{Z}\left[x_{1}, \ldots, x_{n}\right]$ such that $p f_{0} \equiv a$ $\bmod \left(f_{1}, \ldots, f_{r}\right)$ on $X$ with $\operatorname{deg}(p)+d_{0} \leq d_{0} D$ and

$$
\log |a|, \mathrm{h}(p)+\mathrm{h}\left(f_{0}\right) \leq D\left(d_{0} \theta_{X}+\mathrm{h}\left(f_{0}\right)\right) .
$$

This implies that $\left(p f_{0}\right)^{|\boldsymbol{\alpha}|+1} \equiv a^{|\boldsymbol{\alpha}|+1} \bmod \left(f_{1}^{\alpha_{1}+1}, \ldots, f_{r}^{\alpha_{r}+1}\right)$ on $X$. Hence

$$
\frac{1}{f_{0}} \equiv \frac{1}{a^{|\boldsymbol{\alpha}|+1}} p^{|\boldsymbol{\alpha}|+1} f_{0}^{|\boldsymbol{\alpha}|} \bmod \left(f_{1}^{\alpha_{1}+1}, \ldots, f_{r}^{\alpha_{r}+1}\right) \text { on } X \backslash V\left(f_{0}\right) .
$$

Consider the polynomial $r$-form defined over $\mathbb{Z}$ given by $\sigma=p^{|\boldsymbol{\alpha}|+1} f_{0}^{|\boldsymbol{\alpha}|} \tau$. Since $X \backslash$ $V\left(f_{0}\right)$ is a neighborhood of $X \cap V(\boldsymbol{f})$, by Proposition 2.5.

$$
\rho_{X, \boldsymbol{f}}(\omega, \boldsymbol{\alpha})=\frac{1}{a^{|\boldsymbol{\alpha}|+1}} \operatorname{Res}_{X_{\mathbb{C}}}\left[\begin{array}{c}
\sigma \\
\boldsymbol{f}^{\boldsymbol{\alpha}+\mathbf{1}}
\end{array}\right] .
$$


The degree and the length of this polynomial $r$-form are bounded by

$$
\operatorname{deg}(\sigma) \leq \operatorname{deg}(\tau)+(|\boldsymbol{\alpha}|+1) \operatorname{deg}(p)+|\boldsymbol{\alpha}| d_{0} \leq e+(|\boldsymbol{\alpha}|+1) d_{0} D
$$

and

$$
\mathrm{h}_{1}(\sigma) \leq \mathrm{h}_{1}(\tau)+(|\boldsymbol{\alpha}|+1) \mathrm{h}_{1}(p)+|\boldsymbol{\alpha}| \mathrm{h}_{1}\left(f_{0}\right) \leq \mathrm{h}_{1}(\tau)+(|\boldsymbol{\alpha}|+1) D\left(d_{0} \theta_{X}+\mathrm{h}\left(f_{0}\right)\right) .
$$

By Proposition 5.7, there are affine polynomials $\ell_{i} \in \mathbb{Z}[\boldsymbol{x}], i=1, \ldots, n$, with $\mathrm{h}\left(\ell_{i}\right) \leq$ $2 \log (\operatorname{deg}(X))+n \log (2)$ such that, for every subset $I \subset\{1, \ldots, n\}$ of cardinality $r$,

$$
\#\left(X_{\mathbb{C}} \cap \bigcap_{i \in I} V\left(\ell_{i}\right)\right)=\operatorname{deg}(X)
$$

Consider the invertible linear map

$$
\boldsymbol{\ell}: \mathbb{A}_{\mathbb{Q}}^{n} \longrightarrow \mathbb{A}_{\mathbb{Q}}^{n}, \quad \boldsymbol{x} \longmapsto\left(\ell_{1}(\boldsymbol{x}), \ldots, \ell_{n}(\boldsymbol{x})\right)
$$

and set $\widetilde{X}=\boldsymbol{\ell}(X) \subset \mathbb{A}_{\mathbb{Q}}^{n}$. Let $\boldsymbol{m}=\boldsymbol{\ell}^{-1}$ be the inverse map. We have that $\operatorname{det}(\boldsymbol{\ell}) \in$ $\mathbb{Z} \backslash\{0\}$, and it follows from Cramer's formulae that $\operatorname{det}(\ell) \boldsymbol{m} \in \mathbb{Z}^{n \times n}$. Moreover,

$$
\begin{aligned}
\log |\operatorname{det}(\boldsymbol{\ell})|, \operatorname{h}(\operatorname{det}(\boldsymbol{\ell}) \boldsymbol{m}) \leq n \max _{i} \mathrm{~h}\left(\ell_{i}\right)+\log (n !) & \\
& \leq 2 n \log (\operatorname{deg}(X))+2 n^{2} \log (2),
\end{aligned}
$$

where $\mathrm{h}(\operatorname{det}(\boldsymbol{\ell}) \boldsymbol{m})$ stands for the logarithm of the maximum of the absolute values of the entries of this matrix with integer coefficients.

By the invariance of the residue under change of variables (Proposition 2.6),

$$
\rho_{X, \boldsymbol{f}}(\omega, \boldsymbol{\alpha})=\frac{1}{a^{|\boldsymbol{\alpha}|+1}} \operatorname{Res}_{\widetilde{X}_{\mathbb{C}}}\left[\left(\boldsymbol{m}^{*} f_{1}\right)^{\alpha_{1}+1}, \ldots,\left(\boldsymbol{m}^{*} f_{r}\right)^{\alpha_{r}+1}\right] .
$$

Let $\boldsymbol{y}=\left(y_{1}, \ldots, y_{n}\right)$ be a group of $n$ variables, corresponding to the coordinates of the second $\mathbb{A}_{\mathbb{Q}}^{n}$ in 6.14). For each pair of subsets $I, J \subset\{1, \ldots, n\}$ of cardinality $r$, put $\boldsymbol{m}_{I, J}=\left(m_{i, j}\right)_{i \in I, j \in J}$ for the corresponding $r \times r$-submatrix of $\boldsymbol{m}$. Write $\sigma=\sum_{I} q_{I} \mathrm{~d} \boldsymbol{x}_{I}$ with $q_{I} \in \mathbb{Z}[\boldsymbol{x}]$. For each multi-index $J$, set

$$
\widetilde{q}_{J}=\operatorname{det}(\boldsymbol{\ell})^{\operatorname{deg}(\sigma)+r} \sum_{I} \operatorname{det}\left(\boldsymbol{m}_{I, J}\right) \boldsymbol{m}^{*} q_{I} \in \mathbb{Z}[\boldsymbol{y}]
$$

and $\widetilde{\sigma}=\sum_{J} \widetilde{q}_{J} \mathrm{~d} \boldsymbol{y}_{J}$, so that $\boldsymbol{m}^{*} \sigma=\operatorname{det}(\boldsymbol{\ell})^{-\operatorname{deg}(\sigma)-r} \widetilde{\sigma}$. Set also $\widetilde{f}_{i}=\operatorname{det}(\boldsymbol{\ell})^{d_{i}} \boldsymbol{m}^{*} f_{i} \in$ $\mathbb{Z}[\boldsymbol{y}]$. It follows from 6.16 that

$$
\begin{aligned}
\rho_{X, \boldsymbol{f}}(\omega, \boldsymbol{\alpha}) & =a^{-|\boldsymbol{\alpha}|-1} \operatorname{det}(\boldsymbol{\ell})^{-\operatorname{deg}(\sigma)-r+\langle\boldsymbol{\alpha}+\mathbf{1}, \boldsymbol{d}\rangle} \operatorname{Res}_{\widetilde{X}_{\mathbb{C}}}\left[\begin{array}{c}
\widetilde{\sigma} \\
\widetilde{\boldsymbol{f}}^{\boldsymbol{\alpha}+\mathbf{1}}
\end{array}\right] \\
& =a^{-|\boldsymbol{\alpha}|-1} \operatorname{det}(\boldsymbol{\ell})^{-\operatorname{deg}(\sigma)-r+\langle\boldsymbol{\alpha}+\mathbf{1}, \boldsymbol{d}\rangle} \sum_{J} \operatorname{Res}_{\widetilde{X}_{\mathbb{C}}}\left[\begin{array}{c}
\widetilde{q}_{J} \mathrm{~d} \boldsymbol{y}_{J} \\
\widetilde{\boldsymbol{f}}^{\boldsymbol{\alpha}+\mathbf{1}}
\end{array}\right]
\end{aligned}
$$

We next bound the height of each residue in the right-hand side of (6.18). We have that $\operatorname{deg}(\widetilde{X})=\operatorname{deg}(X)$ and, using [KPS01, Lemma 2.7] and [DKS13, Proposition 2.39(5)], the height of $\widetilde{X}$ can be bounded by

$$
\mathrm{h}(\widetilde{X}) \leq \mathrm{h}(X)+(r+1)(2 \log (\operatorname{deg}(X))+n \log (2)+12 \log (n+1)) \operatorname{deg}(X) .
$$

We have that $\operatorname{deg}\left(\widetilde{f}_{i}\right)=d_{i}$ and, by [KPS01, Lemma 1.2(c)] and the bounds in 6.15),

$$
\begin{aligned}
\mathrm{h}\left(\widetilde{f}_{i}\right) & \leq \mathrm{h}\left(f_{i}\right)+d_{i}\left(2 n \log (\operatorname{deg}(X))+2 n^{2} \log (2)+2 \log (n+1)\right) \\
& \leq \mathrm{h}\left(f_{i}\right)+2 n d_{i} \log \left(2^{n+1} \operatorname{deg}(X)\right) .
\end{aligned}
$$


Now fix a multi-index $J$ and let $\widetilde{q}_{J}$ be as in (6.17). We have that

$$
\operatorname{deg}\left(\widetilde{q}_{J}\right) \leq \operatorname{deg}(\sigma) \leq e+(|\boldsymbol{\alpha}|+1) d_{0} D .
$$

Applying again [KPS01, Lemma 1.2(c)] and the bounds in 6.15), we get, for each multi-index $I$ in 6.17),

$$
\mathrm{h}_{1}\left(\operatorname{det}(\boldsymbol{\ell})^{\operatorname{deg}(\sigma)+r} \operatorname{det}\left(\boldsymbol{m}_{I, J}\right) \boldsymbol{m}^{*} q_{I}\right) \leq \mathrm{h}_{1}\left(q_{I}\right)+2 n \log \left(2^{n+1} \operatorname{deg}(X)\right) \operatorname{deg}\left(q_{I}\right) .
$$

Hence, for each $J$,

$$
\begin{aligned}
\mathrm{h}_{1}\left(\widetilde{q}_{J}\right) \leq & \mathrm{h}_{1}(\tau)+(|\boldsymbol{\alpha}|+1) D\left(d_{0} \theta_{X}+\mathrm{h}\left(f_{0}\right)\right)+2 n\left(e+(|\boldsymbol{\alpha}|+1) d_{0} D\right) \log \left(2^{n+1} \operatorname{deg}(X)\right) \\
\leq & \mathrm{h}_{1}(\tau)+2 n e \log \left(2^{n+1} \operatorname{deg}(X)\right) \\
& +(|\boldsymbol{\alpha}|+1)\left(\left(2 n D \log \left(2^{n+1} \operatorname{deg}(X)\right)+D \theta_{X}\right) d_{0}+D \operatorname{deg}(X) \mathrm{h}\left(f_{0}\right)\right) \\
\leq & \mathrm{h}_{1}(\tau)+(2 n+1) e \log \left(2^{n+1} \operatorname{deg} X\right)+(|\boldsymbol{\alpha}|+1) D \kappa_{1}
\end{aligned}
$$

with

$$
\kappa_{1}=d_{0}\left(\frac{\mathrm{h}(X)}{\operatorname{deg}(X)}+\sum_{i=1}^{r} \frac{\mathrm{h}\left(f_{i}\right)}{d_{i}}+2 n \log \left(2^{n+1} \operatorname{deg}(X)\right)+(4 r+8) \log (n+3)\right)+\mathrm{h}\left(f_{0}\right) .
$$

The result follows then from Theorem 6.2. To abridge the rest of the proof, we state the obtained bounds without detailing the involved computations. Set

$$
\kappa_{2}=\frac{\mathrm{h}(X)}{\operatorname{deg}(X)}+\sum_{j=1}^{r} \frac{\mathrm{h}\left(f_{j}\right)}{d_{j}}+2(n+1)^{2} \log (\operatorname{deg}(X))+3(n+2)(n+5) \log (n+2),
$$

which is a quantity that bounds from above the constant $\kappa^{0}$ given by this theorem applied to the variety $\widetilde{X}$ and the $\widetilde{f}_{j}$ 's. Then, this theorem together with the bounds in 6.19) and 6.20 implies that there exists $\zeta_{J} \in \mathbb{Z} \backslash\{0\}$ such that $\zeta_{J} \cdot \rho_{\tilde{X}, \tilde{\boldsymbol{f}}}\left(\widetilde{q}_{J} \mathrm{~d} \boldsymbol{y}_{J}, \boldsymbol{\alpha}\right) \in \mathbb{Z}$ and

$$
\begin{aligned}
\log \left|\zeta_{J}\right|, \log \left|\zeta_{J} \cdot \rho_{\widetilde{X}, \widetilde{\boldsymbol{f}}}\left(\widetilde{q}_{J} \mathrm{~d} \boldsymbol{y}_{J}, \boldsymbol{\alpha}\right)\right| \leq \mathrm{h}_{1}(\tau)+2 n e \log \left(2^{n+1} \operatorname{deg}(X)\right) \\
+(r+1)\left(e D \kappa_{2}+(|\boldsymbol{\alpha}|+1)\left(D \kappa_{1}+\left(d_{0}+r+1\right) D^{2} \kappa_{2}\right)\right) .
\end{aligned}
$$

It follows from (6.18) that we can take the wanted denominator $\zeta \in \mathbb{Z} \backslash\{0\}$ as

$$
\zeta=a^{|\boldsymbol{\alpha}|+1} \operatorname{det}(\boldsymbol{\ell})^{\operatorname{deg}(\sigma)+r-\langle\boldsymbol{\alpha}+\mathbf{1}, \boldsymbol{d}\rangle} \prod_{J} \zeta_{J},
$$

the product being over the subsets $J \subset\{1, \ldots, n\}$ of cardinality $r$. We deduce from (6.11), 6.15) and 6.21) that

$$
\begin{aligned}
& \log |\zeta|+\mathrm{h}_{1}(\tau), \log \left|\zeta \cdot \rho_{X, \boldsymbol{f}}(\omega, \boldsymbol{\alpha})\right| \leq d_{0} D \theta_{X}+D \mathrm{~h}\left(f_{0}\right)+2 n \log \left(2^{n+1} \operatorname{deg}(X)\right) e \\
& \quad+\left(\begin{array}{l}
n \\
r
\end{array}\right)\left(\mathrm{h}_{1}(\tau)+\left(\left(\begin{array}{l}
n \\
r
\end{array}\right)+1\right) 2 n \log \left(2^{n+1} \operatorname{deg}(X)\right) e+(r+1)\left(\begin{array}{l}
n \\
r
\end{array}\right) e D \kappa_{2}+\log \left(\begin{array}{c}
n \\
r
\end{array}\right)\right. \\
& \quad+(|\boldsymbol{\alpha}|+1)\left((r+1)\left(\begin{array}{l}
n \\
r
\end{array}\right)\left(D \kappa_{1}+\left(d_{0}+r+1\right) D^{2} \kappa_{2}\right)+2 n \log \left(2^{n+1} \operatorname{deg}(X)\right) d_{0} D\right) .
\end{aligned}
$$

The bound in Theorem 6.6 is a simpler (and rougher) version of this upper bound.

In the case $X=\mathbb{A}_{\mathbb{Q}}^{n}$, we apply Theorem 6.3 to the residues associated to the polynomial $n$-form $a^{-|\boldsymbol{\alpha}|-1} p^{|\boldsymbol{\alpha}|+1} f_{0}^{|\boldsymbol{\alpha}|} g \mathrm{~d} \boldsymbol{x}=a^{-|\boldsymbol{\alpha}|-1} \sigma$ appearing in 6.12 . With notation as in this result, set

$$
\zeta=a^{|\boldsymbol{\alpha}|+1} \vartheta^{\operatorname{deg}(\sigma)+(|\boldsymbol{\alpha}|+1)(n D+1)} \in \mathbb{Z} \backslash\{0\} .
$$


By Theorem 6.3, we have that $\zeta \cdot \rho_{\mathbb{A}_{\mathbb{Q}}^{n}, \boldsymbol{f}}\left(a^{-|\boldsymbol{\alpha}|-1} \sigma, \boldsymbol{\alpha}\right) \in \mathbb{Z}$ and, using 6.11 and 6.13,

$$
\begin{aligned}
\log |\zeta| & \leq(|\boldsymbol{\alpha}|+1) \log |a|+(\operatorname{deg}(\sigma)+(|\boldsymbol{\alpha}|+1)(n D+1)) \log |\vartheta| \\
& \leq(|\boldsymbol{\alpha}|+1)\left(d_{0} D \kappa+D \mathrm{~h}\left(f_{0}\right)\right)+\left(e+(|\boldsymbol{\alpha}|+1)\left(\left(d_{0}+n\right) D+1\right)\right) n D \kappa \\
& \leq e n D \kappa+(|\boldsymbol{\alpha}|+1)\left(D \mathrm{~h}\left(f_{0}\right)+\left(d_{0}+n\right)(n D+1) D \kappa\right) .
\end{aligned}
$$

with $\kappa=\theta_{\mathbb{A}_{\mathbb{Q}}^{n}}$ as in the statement. Similarly, $\log \left|\zeta \cdot \rho_{\mathbb{A}_{\mathbb{Q}}^{n}, \boldsymbol{f}}\left(a^{-|\boldsymbol{\alpha}|-1} \sigma, \boldsymbol{\alpha}\right)\right|$ is bounded by

$$
\begin{aligned}
\mathrm{h}_{1}(g)+(\operatorname{deg}(\sigma) & +(|\boldsymbol{\alpha}|+1) n D)) n D \kappa+(|\boldsymbol{\alpha}|+1)\left(d_{0} D \kappa+D \mathrm{~h}\left(f_{0}\right)\right) \\
& \leq \mathrm{h}_{1}(g)+e n D \kappa+(|\boldsymbol{\alpha}|+1)\left(D \mathrm{~h}\left(f_{0}\right)+\left(d_{0}+n\right)(n D+1) D \kappa\right),
\end{aligned}
$$

yielding the bounds in Theorem 6.7 .

\section{REFERENCES}

[AY83] I. A. Aı̆zenberg and A. P. Yuzhakov, Integral representations and residues in multidimensional complex analysis, Transl. Math. Monogr., vol. 58, Amer. Math. Soc., 1983.

[BGVY94] C. A. Berenstein, R. Gay, A. Vidras, and A. Yger, Residue currents and Bézout identities, Progress in Mathematics, vol. 114, Birkhäuser, 1994.

[BH97] J.-Y. Boyer and M. Hickel, Une généralisation de la loi de transformation pour les résidus, Bull. Soc. Math. France 125 (1997), 315-335.

[BH99]_ Extension dans un cadre algébrique d'une formule de Weil, Manuscripta Math. 98 (1999), 195-223.

[BPS14] J. I. Burgos Gil, P. Philippon, and M. Sombra, Arithmetic geometry of toric varieties. metrics, measures and heights, Astérisque, vol. 360, Soc. Math. France, 2014.

[BVY05] C. A. Berenstein, A. Vidras, and A. Yger, Analytic residues along algebraic cycles, J. Complexity 21 (2005), 5-42.

[BY99] C. A. Berenstein and A. Yger, Residue calculus and effective Nullstellensatz, Amer. J. Math. 121 (1999), 723-796.

[CH78] N. R. Coleff and M. E. Herrera, Les courants résiduels associés à une forme méromorphe, Lecture Notes in Math., vol. 633, Springer, 1978.

[DKS13] C. D'Andrea, T. Krick, and M. Sombra, Heights of varieties in multiprojective spaces and arithmetic Nullstellensätze, Ann. Sci. Éc. Norm. Supér. (4) 46 (2013), 549-627.

[DP99] S. David and P. Philippon, Minorations des hauteurs normalisées des sous-variétés des tores, Ann. Scuola Norm. Sup. Pisa Cl. Sci. (4) 28 (1999), 489-543.

[Her66] M. Herrera, Integration on a semianalytic set, Bulletin de la Société Mathématique de France 94 (1966), 141-180.

[Hic01] M. Hickel, Solution d'une conjecture de C. Berenstein-A. Yger et invariants de contact à l'infini, Ann. Inst. Fourier (Grenoble) 51 (2001), 707-744.

[Jel05] Z. Jelonek, On the effective Nullstellensatz, Invent. Math. 162 (2005), 1-17.

[KPS01] T. Krick, L. M. Pardo, and M. Sombra, Sharp estimates for the arithmetic Nullstellensatz, Duke Math. J. 109 (2001), 521-598.

[Kun08] E. Kunz, Residues and duality for projective algebraic varieties, Univ. Lecture Ser., vol. 47, Amer. Math. Soc., 2008.

[Poly74] J.B. Poly, Formule des résidus et intersection des chaînes sous-analytiques, Thèse presentée à l'Université de Poitiers. Faculté des Sciences de Poitiers, No. 181. Université de Poitiers, Poitiers, 1974. iii +76 pp.

[SZ16] M. Søgaard and Y. Zhang, Scattering equations and global duality of residues, Phys. Rev. D 93 (2016), 105009.

[Tsi92] A. K. Tsikh, Multidimensional residues and their applications, Transl. Math. Monogr., vol. 103, Amer. Math. Soc., 1992.

[TY04] A. Tsikh and A. Yger, Residue currents, J. Math. Sci. (N. Y.) 120 (2004), 1916-1971. 
Institució Catalana de Recerca i Estudis Avançats (ICREA). Passeig Lluís CompaNYS 23, 08010 BARCELONA, SPAin

Departament de Matemàtiques i Informàtica, Universitat de Barcelona. Gran Via 585, 08007 Barcelona, Spain

E-mail address: sombra@ub.edu

$U R L:$ http://www.maia.ub.edu/ sombra

Institut de Mathématiques, Université de Bordeaux. 351 Cours de la Libération, 33405 Talence, France

E-mail address: Alain.Yger@math.u-bordeaux.fr

$U R L:$ http://www.math.u-bordeaux.fr/ ayger/ 